\title{
Roadmap on Transformation Optics
}

Martin McCall ${ }^{1, *}$, John B Pendry ${ }^{1}$, Vincenzo Galdi ${ }^{2}$, Yun Lai ${ }^{3}$, S. A. R. Horsley ${ }^{4}$, Jensen Li ${ }^{5}$, Jian Zhu ${ }^{5}$, Rhiannon C Mitchell-Thomas ${ }^{4}$, Oscar Quevedo-Teruel ${ }^{6}$, Philippe Tassin ${ }^{7}$, Vincent Ginis ${ }^{8}$, Enrica Martini ${ }^{9}$, Gabriele Minatti ${ }^{9}$, Stefano Maci ${ }^{9}$, Mahsa Ebrahimpouri ${ }^{6}$, Yang Hao ${ }^{10}$, Paul Kinsler ${ }^{11}$, Jonathan Gratus ${ }^{11,12}$, Joseph M Lukens ${ }^{13}$, Andrew M Weiner ${ }^{14}$, Ulf Leonhardt ${ }^{15}$, Igor I. Smolyaninov ${ }^{16}$, Vera N. Smolyaninova ${ }^{17}$, Robert T. Thompson ${ }^{18}$, Martin Wegener ${ }^{18}$, Muamer Kadic ${ }^{18}$ and Steven A. Cummer ${ }^{19}$

\section{Affiliations}

${ }^{1}$ Imperial College London, Blackett Laboratory, Department of Physics, Prince Consort Road, London SW7 2AZ, United Kingdom

${ }^{2}$ Field \& Waves Lab, Department of Engineering, University of Sannio, I-82100 Benevento, Italy

${ }^{3}$ College of Physics, Optoelectronics and Energy \& Collaborative Innovation Center of Suzhou Nano Science and Technology, Soochow University, Suzhou 215006, China

${ }^{4}$ University of Exeter, Department of Physics and Astronomy, Stocker Road, Exeter, EX4 4QL United Kingdom

${ }^{5}$ School of Physics and Astronomy, University of Birmingham, Edgbaston, Birmingham, B15 2TT, United Kingdom

${ }^{6}$ KTH Royal Institute of Technology, SE-10044, Stockholm, Sweden

${ }^{7}$ Department of Physics, Chalmers University , SE-412 96 Göteborg, Sweden

${ }^{8}$ Vrije Universiteit Brussel Pleinlaan 2, 1050 Brussel, Belgium

${ }^{9}$ Dipartimento di Ingegneria dell'Informazione e Scienze Matematiche, University of Siena, Via Roma, 5653100 Siena, Italy

${ }^{10}$ School of Electronic Engineering and Computer Science, Queen Mary University of London, London E1 4FZ, United Kingdom

${ }^{11}$ Physics Department, Lancaster University, Lancaster LA1 4 YB, United Kingdom

${ }^{12}$ Cockcroft Institute, Sci-Tech Daresbury, Daresbury WA4 4AD, United Kingdom

${ }^{13}$ Quantum Information Science Group, Computational Sciences and Engineering Division, Oak Ridge National Laboratory, Oak Ridge, Tennessee 37831, USA

${ }^{14}$ School of Electrical and Computer Engineering, Birck Nanotechnology Center, and Purdue Quantum Center, Purdue University, West Lafayette, Indiana 47907, USA

${ }^{15}$ Physics of Complex Systems, Weizmann Institute of Science, Rehovot 7610001 Israel

${ }^{16}$ Institute for systems research, University of Maryland, College Park, MD 20742, USA

${ }^{17}$ Department of Physics, Astronomy and Geosciences, Towson University, Towson, MD 21252, USA 
${ }^{18}$ Karlsruhe Institute of Technology, Institut für Angewandte Physik, Wolfgang-Gaede-Straße 1, D76131 Karlsruhe, Germany

${ }^{19}$ Electrical and Computer Engineering, Duke University, PO Box 90291, Durham, NC 27708, USA

*Guest editor of the roadmap

Email: m.mccall@imperial.ac.uk

\begin{abstract}
Transformation Optics asks Maxwell's equations what kind of electromagnetic medium recreate some smooth deformation of space. The guiding principle is Einstein's principle of covariance: that any physical theory must take the same form in any coordinate system. This requirement fixes very precisely the required electromagnetic medium.

The impact of this insight cannot be overestimated. Many practitioners were used to thinking that only a few analytic solutions to Maxwell's equations existed, such as the monochromatic plane wave in a homogeneous, isotropic medium. At a stroke, Transformation Optics increases that landscape from 'few' to 'infinity', and to each of the infinitude of analytic solutions dreamt up by the researcher, corresponds an electromagnetic medium capable of reproducing that solution precisely.
\end{abstract}

The most striking example is the electromagnetic cloak, thought to be an unreachable dream of science fiction writers, but realised in the laboratory a few months after the papers proposing the possibility were published. But the practical challenges are considerable, requiring meta-media that are at once electrically and magnetically inhomogeneous and anisotropic. How far have we come since the first demonstrations over a decade ago? And what does the future hold? If the wizardry of perfect macroscopic optical invisibility still eludes us in practice, then what compromises still enable us to create interesting, useful, devices?

While 3D cloaking remains a significant technical challenge, much progress has been made in 2dimensions. Carpet cloaking, wherein an object is hidden under a surface that appears optically flat, relaxes the constraints of extreme electromagnetic parameters. Surface wave cloaking guides subwavelength surface waves, making uneven surfaces appear flat. Two dimensions is also the setting in which conformal and complex coordinate transformations are realisable, and the possibilities in this restricted domain do not appear to have been exhausted yet.

Beyond cloaking, the enhanced electromagnetic landscape provided by Transformation Optics has shown how fully analytic solutions can be found to a number of physical scenarios such as plasmonic systems used in electron energy loss spectroscopy (EELS) and cathodoluminescence (CL). Are there further fields to be enriched?

A new twist to Transformation Optics was the extension to the space-time domain. By applying transformations to space-time, rather than just space, it was shown that events rather than objects could be hidden from view; Transformation Optics had provided a means of effectively redacting events from history. The hype quickly settled into serious nonlinear optical experiments that demonstrated the soundness of the idea, and it is now possible to consider the practical implications, particularly in optical signal processing, of having an 'interrupt-without-interrupt' facility that the socalled temporal cloak provides. Inevitable issues of dispersion in actual systems have only begun to be addressed.

Now that time is included in the programme of Transformation Optics, it is natural to ask what role ideas from General Relativity can play in shaping the future of Transformation Optics. Indeed, one of 
the earliest papers on Transformation Optics was provocatively titled 'General Relativity in Electrical Engineering'. The answer that curvature does not enter directly into transformation optics merely encourages us to speculate on the role of Transformation Optics in defining laboratory analogues.

Quite why Maxwell's theory defines a 'perfect' transformation theory, while other areas of physics such as acoustics are not apparently quite so amenable, is a deep question whose precise, mathematical answer will help inform us of the extent to which similar ideas can be extended to other fields.

The contributors to this roadmap review, who are all renowned practitioners or inventors of Transformation Optics, will give their perspectives into the field's status and future development.

\section{Contents}

1. Introduction

2. Near Field Transformation Optics

3. Spatial dispersion and Spectral Domain Transformation Optics 3.1 Nonlocal and non-Hermitian extensions of transformation optics

3.2 Transformation optics by photonic crystals: a nonlocal route towards the optical frequency regime

4. Complexification in Transformation Optics

5. Surface Transformation Optics

5.1 Extending transformation optics and towards a thin cloak

5.2 Combining curvature and index gradients for surface and guided waves

5.3 Transformation optics at surfaces

6. Antennas

6.1 Metasurface Radiation and Guidance at Microwaves

6.2 Bespoke lenses: An opportunity for tailoring antenna radiation patterns

6.3 Transformation Optics for Antenna Engineering

7. Spacetime Cloaking

7.1 Spacetime Transformation Optics

7.2 Transformations optics with spatial dispersion

7.3 Experimental progress in temporal cloaking

8. Transformation Optics for Analogue Cosmology

8.1 Cosmology in the laboratory: challenges at the horizon

8.2 Spacetime analogs based on hyperbolic metamaterials

8.3 Transformation optics in general relativity

9. Optics and Beyond

9.1 Seeking applications in optics and beyond

9.2 Beyond Optics: Transforming Other Wave And Transport Systems 


\section{Introduction - Martin McCall}

Imperial College

A little over a decade ago Pendry et al noted that the long-known form-invariance of Maxwell's equations could be interpreted as an extremely flexible design recipe for achieving virtually arbitrary distortions of electromagnetic fields. It is an unusual theory in that the recipe is exact, i.e. all aspects of the electromagnetic field, amplitude, phase and polarization are in principle relocated by an appropriate material design. The method was immediately applied to design and build a device that guided light around a void region in space, thus bringing the celebrated invisibility cloak of science fiction right into the laboratory. Although the cloak is the most striking example, transformation optics has since developed many other applications that combine its design ingenuity with parallel developments in metamaterials technology. In this Roadmap review several leading practitioners give their unique personal perspectives of the current status of the field, and where the future challenges lie.

Pendry's article focuses on the near-field and the impact of TO on surface plasmonics, explaining, for example, the huge enhancements of local fields around structural singularities, and the resultant opportunities offered to spectroscopy and nonlinear optics resulting from the very tight compression of light fields.

Transformation Optics designs invariably prescribe inhomogeneous material parameters. A striking departure from this is described by Vincenzo Galdi, who examines the use of the TO algorithm in the spectral domain. Such designer dispersion prescribes media with fascinating frequency dependencies, all the while maintaining uniformity in the spatial domain. The theme of spatial dispersion in TO is taken up again in Lai's article, who discusses how photonic band-gap engineering is combined with TO to produce omnidirectional impedance matching.

Simon Horsley shows how analytic continuation of the coordinates into the complex arena permits extensions to media with gain and loss, connecting TO closely with so-called PT symmetric media, and showing how to design reflectionless media. Rather than exploit form invariance under coordinate transformation, Li et al explain how new possibilities emerge if, instead, form invariance is pursued with respect to transformation of the electromagnetic field, leading to, for example, an effective magnetic bending force on light. They also discuss strategies for producing thin cloaks based on metasurfaces.

Rhiannon Mitchell-Thomas reviews geometrical approaches confined to curved surfaces, rather than 3D space, showing that TO designs using isotropic graded index media allow for novel implementations of, for example, Eaton and Luneburg lenses.

Discontinuous transformations at surfaces are discussed by Philippe Tassin et al, who show how the latest metasurface technology opens up applications in integrated optics.

Martini et al discuss how a planar version of Transformation Optics leads to control of surface/plasmonic wave propagation or 'metasurfing'; while Oscar Quevedo-Tereul shows how TO is being used to tailor antenna radiation patterns using bespoke lenses. The theme of antenna engineering is taken up by Yang Hao in reviewing how TO based radome designs are leading to novel compact phased arrays.

\section{Paul Kinsler and Jonathan Gratus consider} generalization of TO to the spacetime domain. Kinsler's article discusses spacetime cloaking, in wich events, not objects, are hidden from view. Gratus shows that in the broader TO regime with both time and space included, spatial dispersion plays a necessary and fundamental role. Lukens et al describe some of the experimental efforts in event cloaking, emphasising the role played by space-time duality in achieving localized shadowing in space-time. They emphasise that applications will likely focus on specific communication tasks, and offer tantalising connections between temporal cloaking and quantum information processing.

As Ulf Leonhardt points out in his contribution, following the detection of gravitational waves, cosmology has now entered a golden era, and how Electromagnetism might repay its debt to GR becomes an issue addressable by TO. Smolyaninov et al discuss, for example, how the mapping between metric and material parameters, a key idea in TO, informs cosmology in the optics lab, with inhomogeneous hyperbolic metamaterials directly analogising signature changing events and multiverses. As Thompson makes clear, the distinction between medium and spacetime contributions in Maxwell's equations opens up several 
potential new avenues since the mapping between distortion and effective medium is actually not unique.

Beyond optics, Martin Wegener takes us from using TO to improve solar cell efficiency to transformation mechanics, showing how mechanical cloaks can improve the utility of scaffolds. Steve Cummer also assesses how the TO algorithm can and has been successfully extended to other disciplines such as acoustics, elastodynamics and heat flow.

A recurring theme in all the contributions is the extant gap between design aspiration and technological feasibility. Theorists have provided beautiful design tools that can potentially give us many very useful and practical devices, but we are frustrated at often only being able to realise a fraction of this potential due to the difficulty of producing broad-band metamaterial designs requiring sub-wavelength fabrication. The compromises currently made on the altar of progress do not do justice to the original designs. Perhaps the main 'take-home' message of this review is to provide a rallying call to technologists and experimentalists to make the necessary step change to meet these challenges and turn, as Cummer notes, simple feasibility demonstrations towards practically useful devices. 


\section{Near Field Transformation Optics - JB \\ Pendry \\ Imperial College London}

\section{Status}

Transformation optics (TO) has its origins in Einstein's general theory of relativity: he showed how Maxwell's equations can be written in a non-Cartesian space and how changes to the coordinate system can be represented as changes to the constitutive parameters, $\varepsilon, \mu$. These ideas were slow to be adopted by the optics community but in 1996 Ward and Pendry [1] faced the challenge of adapting electromagnetic finite difference codes to the cylindrical geometry of a fibre. Originally written in Cartesian coordinates to tackle photonics crystals the codes did not need to be reprogrammed in cylindrical coordinates, but simply presented with a transformed $\boldsymbol{\varepsilon}, \boldsymbol{\mu}$. However the most powerful insight came with the realisation that under a coordinate transformation lines of force, whether electric or magnetic, remained attached to the coordinate system. A picture emerged of space as a deformable entity, and the possibility of rearranging the fields by distorting space, then asking through Einstein's equations what values of $\varepsilon, \mu$ would send the fields in the desired direction. The value of this insight is that it replaces the intuitive pictures of ray optics with a way to manipulate field lines, but retaining the insight offered by a physical picture of what is going on. Furthermore an intuitive picture can be given of how $\varepsilon, \mu$ transform. Under a simple compression by $\alpha$ of coordinates along one axis, components of both $\varepsilon, \mu$ along that axis are reduced by a factor of $\alpha$; components perpendicular to the axis are increased by $\alpha^{-1}$. Any transformation can be represented by a series of compressions and rotations and thus the whole transformation can be understood in geometrical terms. A review of these ideas can be had in reference [2].

These ideas first gained attention when deployed to design a cloak of invisibility, a challenge that is almost impossibly difficult using conventional paradigms but ridiculously simple for TO. Many cloaks can also be envisaged in terms of deflected rays, but this picture is not available to us for structures that are much less than the wavelength and we are confronted with the so called 'near fields' where the electric and magnetic components are no longer tightly coupled into the ray picture but lead a more independent existence. For example a cloak for static magnetic fields can in no way be envisaged as controlling a set of rays and we are forced instead to think of lines of force. Such a cloak has been realised by Supradeep Narayana and Yuki Sato [3] and figure 1 shows their results.

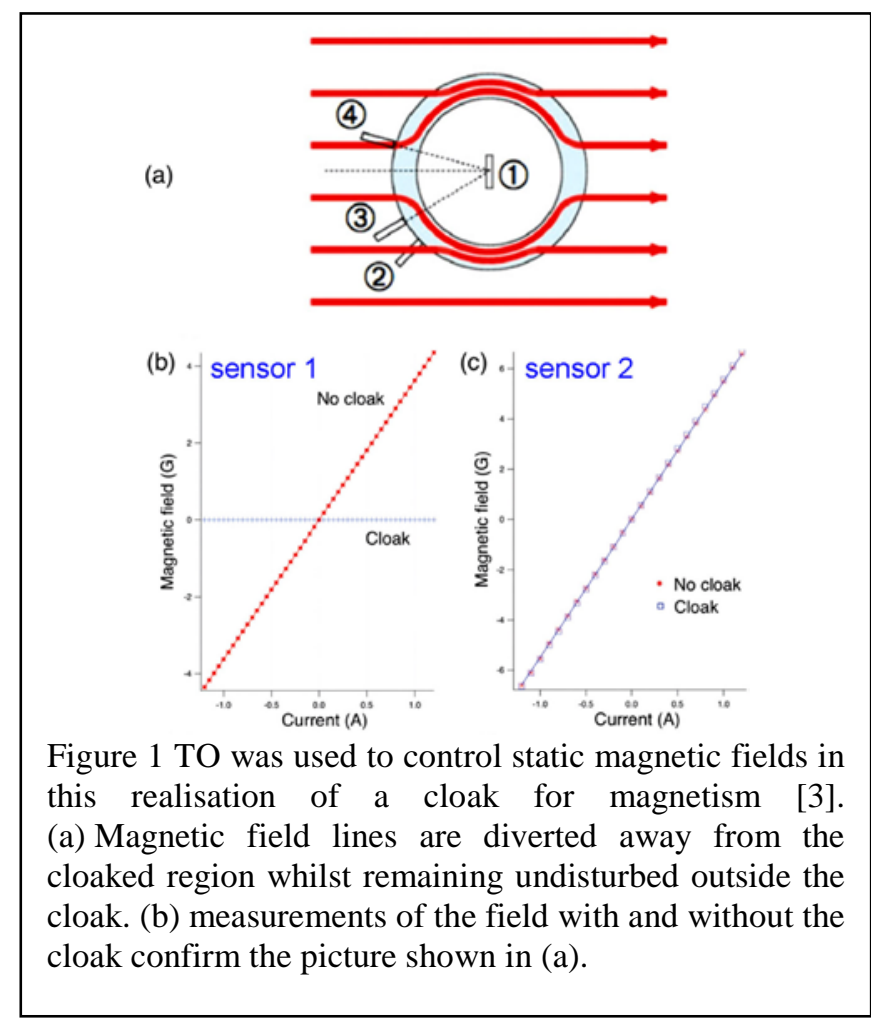

TO has had a strong impact on plasmonic systems. The optical response of metals is dominated by the sea of free electrons and on the surface the electron sea supports electromagnetic waves called surface plasmons. Bound to the metal surface and with a wavelength much shorter than that of light in free space, their behaviour is dominated by the shape of the surface. For example, a highly polished silver surface is an excellent mirror reflecting nearly all incident light. On the other hand finely divided silver in the form of nanoparticles, such as found in photographic negatives, absorbs nearly all incident light and is black. This is an extreme example of a metamaterial: internal structure dominates the optical response. Understanding the interplay of surface structure with the near field has enabled new insights.

\section{Current and Future Challenges}

Surface plasmonics [4] is perhaps the field in which TO has most impact. Plasmonics burst spectacularly into spectroscopy when Fleischman made the observation that Raman signals from molecules adsorbed on a rough silver surfaces were many orders of magnitude greater than the free space cross sections would predict. The effect can be explained by huge enhancements of local fields of incident light around structural singularities. Heavy computational studies confirmed this explanation but provided little insight into why singularities produce enhancement. It fell to transformation optics to explain the effect in simple terms. 
Consider a structure consisting of a surface containing a sharp knife edge. The edge constitutes a singularity and local electric fields are massively enhanced in its vicinity when the structure is illuminated. The singularity can be removed by a simple transformation,

$$
\begin{aligned}
& x^{\prime}=\ln \sqrt{x^{2}+y^{2}} \\
& y^{\prime}=\arctan y / x
\end{aligned}
$$

where the origin is located on the edge. This transforms the edge to a non singular array of wave guides and the singularity at the origin of $\boldsymbol{x}, \boldsymbol{y}$ space vanishes to infinity in $x^{\prime}, y^{\prime}$ space. All singular structures can have their singularities removed in this fashion. Enhancements can be understood as the compression of an infinite system into a finite one. This has the curious consequence that a finite singular structure has a continuous spectrum typical of an infinite system, and an infinity in the density of states at the singularity. Though this result has to be qualified for real materials where practical considerations of loss and of non locality of their response can reduce predicted enhancements. More details can be had in reference [5].

Another system described in [5] is shown in figure 2. In this instance the transformation is an inversion which takes a simple metallic waveguide and transforms it into two touching cylinders. The transformation is singular at the touching point which produces intense local fields. Not only does a transformation explain why local fields are so intense, it also provides a means of classifying the spectrum: often it is the case that the transformed system, lacking the original singularity, has a simpler structure. In the examples given above the transformed waveguide structure is translationally invariant along the $\boldsymbol{x}^{\boldsymbol{\prime}}$ axis allowing modes to be classified as Bloch waves, assigned a wave vector, $k$, and their frequencies calculated analytically as a function of $\boldsymbol{k}$.

TO can also be applied in the context of the refractive index to remove singularities from a structure as has been elegantly demonstrated in reference [6]

\section{Advances in Science and Technology to Meet Challenges}

Several challenges meet these beautiful theoretical concepts when inviting experimentalists into the field. The first is the materials challenge. Most of the conclusions presented above assume an ideal loss free metal described by a local dielectric function, $\boldsymbol{\varepsilon}$. Locality means that $\varepsilon$ depends only on frequency and not on wave vector. Loss absorbs energy and prevents realisation of ideal field enhancements, so that only relatively loss free metals such as silver and gold are serious candidates for the effects. Non locality has the effect of smearing out any singularities in the surface structure. In metals non locality arises because the electron sea has limited compressibility and cannot be squeezed into very sharp corners preventing realisation of an ideal singularity $[7,8,9]$. The search is on for new materials that perform even better than our current optimum candidates of silver and gold [10], a challenge made more difficult by our limited theoretical understanding of losses and non locality beyond the standards achieved by current best performers.

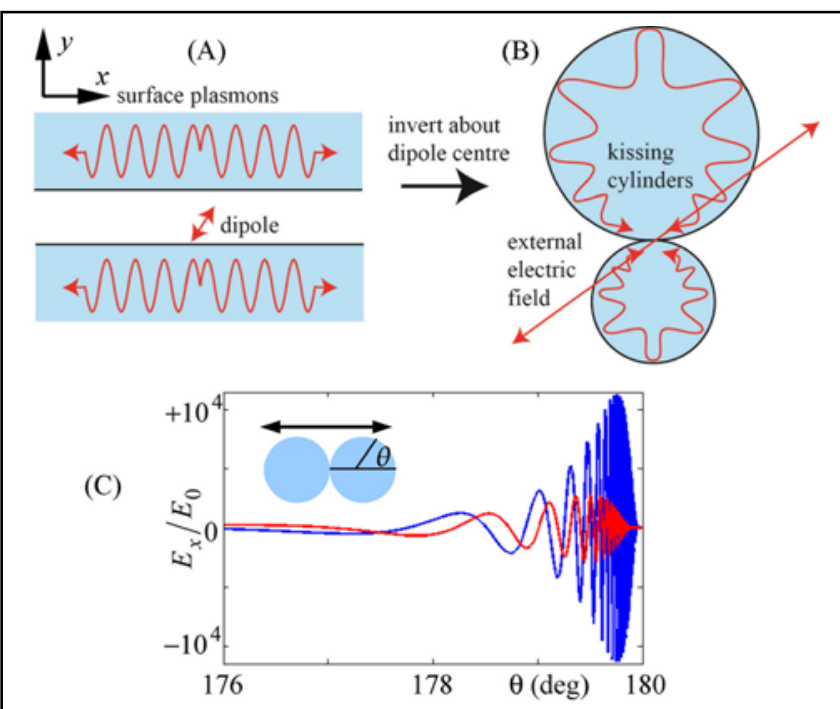

Figure 2 (A) Surface plasmons are excited by light emitted from an excited atom in a metallic waveguide. (B) Transformation by inversion about the atom centre generates a completely different structure of two touching cylinders. (C) The waveguide solutions from (A) map directly to the touching cylinders giving strongly enhanced fields near the point of contact.

Beyond that there is the issue of how to sculpt surfaces to the required geometrical perfection. Metals have very high surface energy and object to the formation of singularities: sharp edges tend to round off, and singularities formed by touching curves often result in the fusing of the two surfaces. Engineering on the nanoscale demands expensive apparatus to achieve the precision needed.

Despite these slightly pessimistic observations great opportunities are to be had. I have alluded to the spectacular enhancements in spectroscopic sensitivity already a reality. Utilising the design tool of TO structures can be optimised to give the best response and single molecule sensitivity can be had.

It is already possibility to compress light into a focus far smaller than the free space Abbé limit. Compression into a square nanometre is possible, many orders of magnitude more dense than achieved with conventional lenses thus achieving with modest power inputs energy densities great enough to trigger 
non linear effects. A cornucopia of new experiments awaits exploitation of nonlinearity, from simple switching of light with light to more sophisticated concepts such as phase conjugation and time reversal.

\section{Concluding Remarks}

TO is a general tool that provides a new way to visualise electromagnetic phenomena, whilst retaining accuracy at the level of Maxwell's equations. It has particular value for the near field where conventional ray pictures are of no use, but where the concept of fields still applies. In the above two areas of application are described: to DC fields where the entire system exists in the near field, and surface plasmonics where most of the interesting phenomena are observed on length scales smaller than the free space wavelength.

Acknowledgments This work was supported by the EPSRC (Grant No. EP/L024926/1), and the Gordon and Betty Moore Foundation.

\section{References}

[1] Ward AJ, and Pendry JB, 1996 Refraction and Geometry in Maxwell's Equations Journal of Modern Optics, 43 773-93

[2] Pendry JB, Luo Y, and Zhao Rongkuo 2015 Transforming the optical landscape Science 348 521-4

[3] Supradeep Narayana and Yuki Sato 2012 DC Magnetic Cloak Advanced Materials 24 71-74

[4] Maier SA 2007 Plasmonics: Fundamentals and Applications (Springer, New York)

[5] Pendry JB, Aubry A, Smith DR, and Maier SA 2012 Transformation optics and subwavelength control of light Science 337, 549-52.

[6] Horsley SAR, Hooper IR, Mitchell-Thomas RC and Quevedo-Teruel O 2014 Removing singular refractive indices with sculpted surfaces Scientific Reports 44876

[7] Mortensen NA, Raza S, Wubs M, Søndergaard T and Bozhevolnyi SI 2014 A generalized non-local optical response theory for plasmonic nanostructures Nature Communications 5, 3809.

[8] Zhu W, Esteban R, Borisov AG, Baumberg JJ, Nordlander P, Lezec HJ, Aizpurua J and Crozier KB 2016 Quantum mechanical effects in plasmonic structures with subnanometre gaps Nature Communications 7, 11495.

[9] Luo Y, Fernandez-Dominguez AI, Wiener A, Maier SA, and Pendry JB 2013 Surface Plasmons and Nonlocality: A Simple Model Physical Review Letters, 111, 093901

[10] Brown AM, Sundararaman R, Narang P, Goddard III WA, and Atwater HA 2016 Nonradiative Plasmon Decay and Hot Carrier Dynamics: Effects of Phonons, Surfaces, and Geometry ACS Nano 10 957-966 


\section{Spatial Dispersion and Spectral Domain Transformation Optics}

\subsection{Nonlocal and non-Hermitian extensions of} transformation optics - Vincenzo Galdi

University of Sannio

\section{Status}

By interpreting the material effects on wave propagation as local distortions of a given coordinate reference frame, transformation optics (TO) provides a very powerful and systematic framework to precisely manipulate electromagnetic fields [1]. In essence, the conceptual design of a desired response may be conceived by relying primarily on geometrical intuition based on the geodesic path of light rays, and is effectively decoupled from the actual material synthesis problem, which is eventually posed as a suitable approximation of some analytically derived ideal constitutive "blueprints".

Conventional TO schemes typically rely on local, realvalued coordinate transformations, which inherently imply some limitations in the attainable fieldmanipulation effects. Thus, typical constitutive blueprints tend to be spatially inhomogeneous and anisotropic [1], whereas it is generally not possible to extrinsically generate nonlinear, nonreciprocal, nonlocal (i.e., spatial dispersion), and non-Hermitian (i.e., loss and/or gain) effects, among others. Several extensions have been proposed in order to overcome these limitations. Here, we focus on certain extensions that enable the TO formalism to deal with nonlocal and non-Hermitian effects, which are becoming increasingly relevant in many metamaterial applications.

\section{Current and Future Challenges}

In [2,3], our team laid out the foundations for a nonlocal TO extension, which can be exploited to engineer complex spatial-dispersion effects. Such extension relies on a reformulation of TO in the frequencywavevector phase space, accessed via temporal and spatial Fourier transforms, and retains the attractive features of conventional TO in terms of decoupling the actual metamaterial synthesis from the conceptual design, with this latter still heavily inspired by geometrical intuition. As illustrated in Fig. 1, a broad variety of interesting dispersion effects can be interpreted in the phase space as geometrical transformations of the conventional conical dispersion surface of a homogeneous medium. Thus, for instance, effects such as one-way (nonreciprocal) propagation, additional extraordinary waves, and slow-light can be associated to analytic properties of the transformation such as non-centersymmetry, multivaluedness, and stationary inflection points, respectively. Similar to the conventional (local) TO approach, our proposed nonlocal extension systematically yields, in analytic closed-form expressions, the required (nonlocal) constitutive blueprints in terms of wavevectordependent permittivity and permeability tensors $[2,3]$.
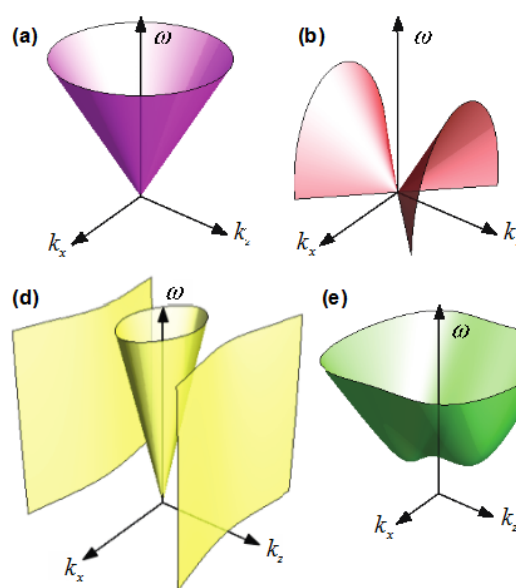

(e)
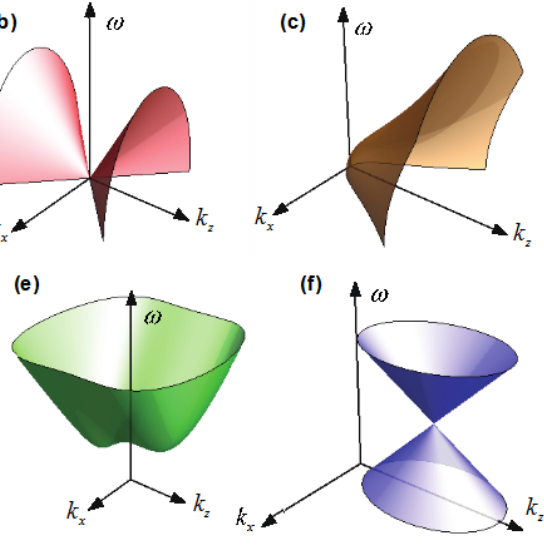

(f) $+\omega$
Figure 1 - Conceptual illustration of the nonlocal TO extension in the frequency $(\boldsymbol{\omega})$-wavevector $\left(k_{x}, k_{z}\right)$ phase space (see [3]). (a) Conical dispersion surface of a homogeneous medium. (b) Hyperbolic dispersion (purely imaginary transformation component). (c) One-way, nonreciprocal propagation (non-centersymmetric transformation). (c) Additional extraordinary wave (multivalued transformation). (d) Frozen mode (stationary inflection point). (f) Dirac-point conical singularity (phasespace shift).

By comparison with conventional (local, spatialdomain) TO, we note that the above nonlocal extension inherently yields spatially homogeneous constitutive blueprints. In view of the underlying spatial Fourier transform, the spectral resolution, i.e., the capability to tailor the dispersion surfaces in the frequencywavevector phase space, is gained at the expense of the spatial resolution. On the other hand, conventional TO allows fine tailoring of the spatial response, but completely lacks spectral resolution. Thus, conventional and nonlocal TO can be viewed as two extremes in the spatial vs. spectral resolution tradeoff. As a future challenge, it would be very intriguing to bridge these two extremes, so as to blend in a desired fashion both spatial and spectral resolution. From the mathematical viewpoint, windowed spatial Fourier transforms, such as the Gabor transform, seem to constitute a particularly suitable, self-consistent framework for such a hybrid TO formulation. In such a scheme, the window width would set the spectral vs. spatial resolution tradeoff via an uncertainty relation, and the conventional and nonlocal TO formulations would be recovered as the limits for zero and infinite window width, respectively. The above-envisioned Gabor-type formulations may 
considerably expand future prospects for TO applications, encompassing complex scenarios featuring, e.g., nonlocal effects at different spatial scales, such as photonic "hypercrystals". Moreover, they may also set the stage for disruptive fieldmanipulation platforms combining the spatial-routing (e.g., multiplexing, steering) capabilities of conventional TO with the inherent signal-processing capabilities (e.g., convolution, differentiation, integration) enabled by nonlocal TO. This may open up new intriguing venues in the emerging field of “computational metamaterials" [4].

In [5,6], our team put forward a complex-coordinate TO extension that can naturally handle non-Hermitian metamaterials featuring spatial modulation of loss and gain. Also in this case, the appealing characteristics of conventional (real-valued) TO in terms of physically incisive modeling and geometry-driven intuitive design are retained, by combining the approach with wellestablished analytic formalisms based, e.g., on the "complex-source-point" (CSP) representation of beamlike wave objects, and the "leaky-wave" (complex propagation constant) modeling of radiating states $[5,6]$. For instance, as schematized in Fig. 2, a complex coordinate transformation relating a "source" and an "image" CSP can be interpreted in the real, physical space as an input-output transformation of an optical beam, induced by a non-Hermitian metamaterial slab, with full control on the waist position, direction and diffraction length. This represents a rather versatile and insightful approach, which brings about new degrees of freedom and may provide new perspectives in the design of active optical devices and radiating systems, with the use of gain not merely limited to achieve loss compensation.

Metamaterials mixing loss and gain are also receiving a surge of interest as potentially feasible testbeds for studying phenomena and properties that are distinctive of non-Hermitian systems. These include parity-time symmetry and exceptional points (i.e., degeneracies between complex eigenstates), which are ubiquitous in many disciplines, ranging from quantum mechanics to nuclear physics [7]. The application of non-Hermitian TO extensions to these scenarios is still largely unexplored, and represents one of the most fascinating developments to pursue. Preliminary studies on paritytime-symmetric metamaterials [5] have shown intriguing connections between the onset of spontaneous symmetry breaking (an example of exceptional point) and the discontinuous character of the complex-coordinate transformation. This may be indicative of deeper connections between the analytic properties of the transformations and the physics of exceptional points, whose exploration may uncover novel effects and shed new light on the phenomenological understanding.

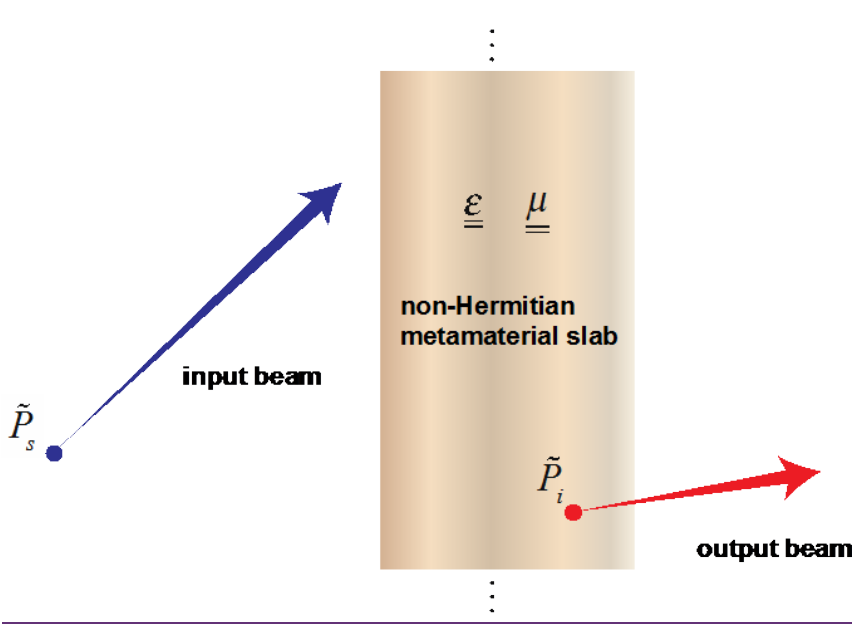

Figure 2 - Schematic representation of the complexcoordinate TO mapping between a "source" and an "image" CSP ( $\tilde{P}_{s}$ and $\tilde{P}_{i}$, respectively). In the physical, real space the mapping can be interpreted as a transformation of an input beam into an output beam (blue and red arrows, respectively), induced by a non-Hermitian metamaterial slab.

\section{Advances in Science and Technology to Meet Challenges}

Nonlocal TO extensions crucially rely on nonlocal effective models to approximately match the desired (ideal) constitutive blueprints with that of a physical metamaterial structure within appropriate phase-space regions. Therefore, essential to further advances in this field is the availability of a comprehensive "library" of effective nonlocal models, building up on general [8] and structure-specific [9] nonlocal homogenization approaches. Moreover, the discovery of new structures and regimes where nonlocal effects are enhanced, and possibly attainable via moderate-permittivity, low-loss material constituents may significantly boost the practical applicability of nonlocal TO. Finally, the development of full-wave numerical simulation tools implementing nonlocal constitutive relationships would also represent a major catalyst.

Non-Hermitian TO, on the other hand, critically relies on the possibility to precisely tailor the spatial distribution of loss and gain, which is becoming technologically viable. At microwave frequencies, gain may be attained via classical amplification schemes based on operational amplifiers and Gunn diodes, whereas at optical wavelengths active dopants such as two-level atoms (or quantum dots) may be exploited. While certain non-Hermitian effects may require unfeasibly high values of gain, it is also important to remark that completely passive (i.e., lossy) structures have been exploited to experimentally demonstrate 
pivotal proof-of-concept effects in the physics of exceptional points (see, e.g., Ref. [10]). Within this framework, metamaterials with near-zero constitutive parameters (real part) [11], appear particularly attractive in view their well-known capabilities to dramatically enhance the effects of relatively low levels of loss and/or gain.

\section{Concluding Remarks}

In a variety of metamaterial applications of current interest, spatial dispersion and loss/gain are escalating from second-order nuances to instrumental effects. Nonlocal and non-Hermitian extensions of the TO approach potentially constitute an attractive framework to harness these effects in a versatile, systematic and insightful fashion.

Further perspectives on nonlocal and non-Hermitian TO extensions are discussed in Sections 3.2, 7.2 and Section 4 , respectively.

\section{References}

[1] Pendry J B, Schurig D and Smith D R 2006 Controlling electromagnetic fields Science $\mathbf{3 1 2}$ 1780-1782

[2] Castaldi G, Galdi V, Alù A and Engheta N 2012 Nonlocal transformation optics Phys Rev Lett 108 063902

[3] Moccia M, Castaldi G, Galdi V, Alù $A$ and Engheta N 2016 Dispersion engineering via nonlocal transformation optics Optica 3 179-10

[4] Silva A, Monticone F, Castaldi G, Galdi V, Alù A and Engheta N 2014 Performing mathematical operations with metamaterials Science 343 160-163.

[5] Castaldi G, Savoia S, Galdi V, Alù $A$ and Engheta N 2013 PT metamaterials via complex-coordinate transformation optics Phys Rev Lett 110173901

[6] Savoia S, Castaldi G and Galdi V 2016 Complexcoordinate non-Hermitian transformation optics $J$ Opt 18 1-13

[7] Heiss W D 2012 The physics of exceptional points $J$ Phys A 45, 444016.

[8] Silveirinha M G 2007 Metamaterial homogenization approach with application to the characterization of microstructured composites with negative parameters Phys Rev B 75115104

[9] Chebykin A V, Orlov A A, Vozianova A V, Maslovski S I, Kivshar Y S and Belov P A 2011 Nonlocal effective medium model for multilayered metal-dielectric metamaterials Phys Rev B 201184 115438

[10] Doppler J, Mailybaev A A, Böhm J, Kuhl U, Girschik A, Libisch F, Milburn TJ, Rabl P, Moiseyev N and Rotter S 2016 Dynamically encircling an exceptional point for asymmetric mode switching Nature 537 7679

[11] Liberal I and Engheta N 2017 Near-zero refractive index photonics Nat Photon 11 149-158 
3.2 Transformation optics by photonic crystals: a nonlocal route towards the optical frequency regime - Yun Lai

Soochow University

\section{Status}

Transformation optics (TO) theory was originally derived in a local medium framework [1,2]. Since coordinate transformation often leads to unusual materials parameters absent in nature, metamaterials [3] have been applied to realize these parameters in novel TO applications, such as cloaking [1-3] and illusion optics [4]. Most of the experimental demonstrations of TO were achieved by metamaterials. However, at optical frequencies, metamaterials suffer from the loss in their metallic components. Moreover, optical metamaterials demand nano-scale unit structures, which makes the fabrication of macroscopic samples quite challenging.

On the other hand, photonic crystals [5] can provide an excellent low-loss platform for optical devices. Besides the famous band-gap properties, photonic crystals are also well known for exhibiting novel refractive behaviors such as negative refraction. Interestingly, negative refraction was first proposed and realized in the field of metamaterials, as a direct result of the double negative parameters. However, negative refraction was also realized by photonic crystals later. Unlike metamaterials that use unusual medium parameters to control the propagation of waves, photonic crystals utilize their spatial dispersions, which can be described by the equal frequency contours (EFCs) in the band structure, to control the wave propagation. By designing specific EFCs, the refractive behaviors of TO media and some novel TO applications can also be simulated by photonic crystals. For instance, Urzhumov and Smith first proposed to utilize elliptical EFCs to simulate the stretched TO media, and thus further realize cloaking phenomena [6]. Liang and $\mathrm{Li}$ proposed to use photonic crystals to replace the TO media in bending waveguides [7]. These seminal studies, however, didn't explore the possibility of realizing the omnidirectional impedance matching condition, which is another distinct signature of the TO media obtained by coordinate transformation. In fact, if omnidirectional impedance matching can also be achieved by pure dielectric photonic crystals, the realization of ideal nonreflecting TO applications in the optical frequency regime is possible.

\section{Current and Future Challenges}

The TO theory can provide the perfect solution of omnidirectional impedance matching in the local

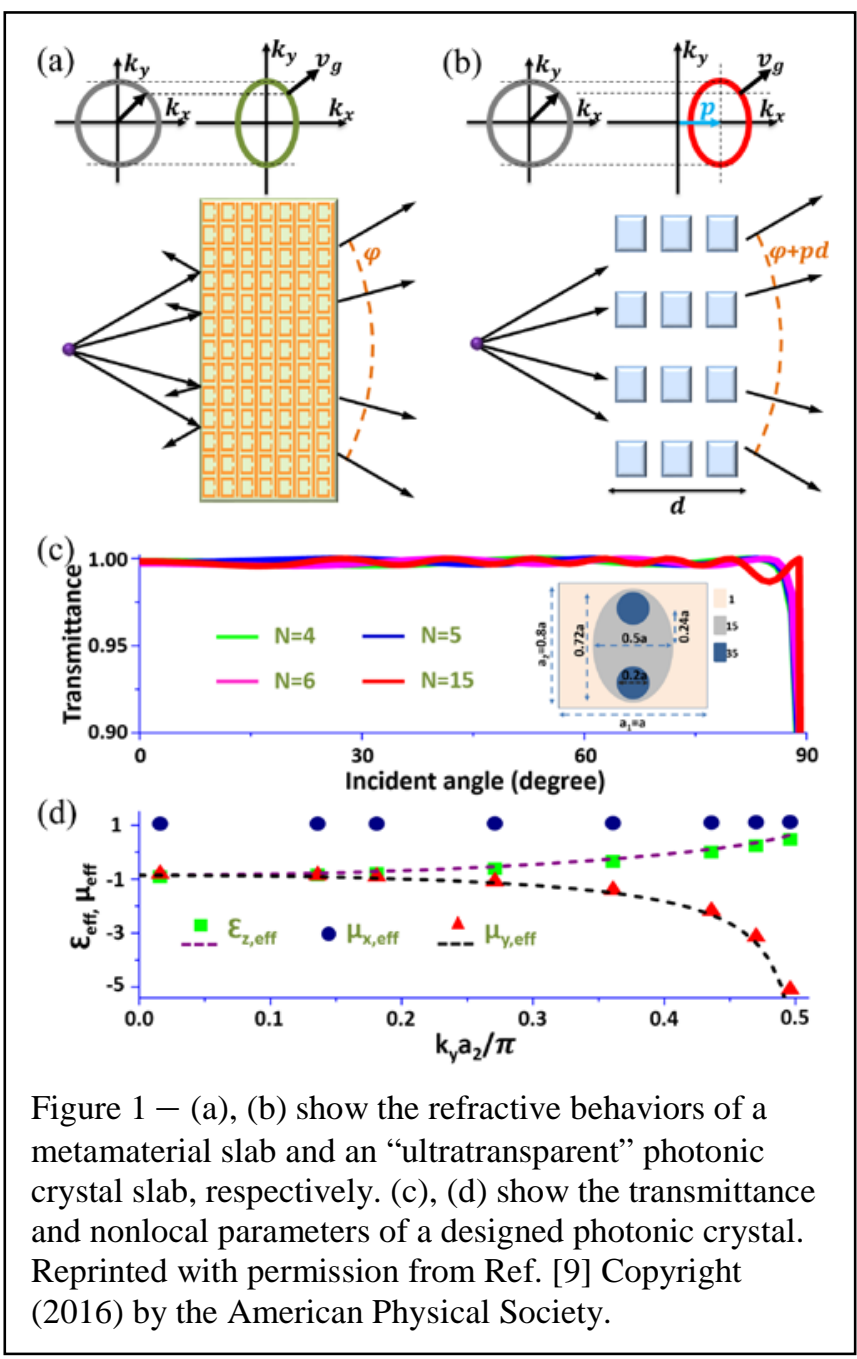

medium framework. However, the solutions usually require complex permittivity and permeability which are difficult to realize. Even in microwave frequencies, the perfect TO parameters are usually reduced significantly to ease the fabrication and the property of omnidirectional impedance matching is normally sacrificed [3]. This led to reflection in cloaking experiments, compromising the ideal invisibility.

Interestingly, it is seldom known that beside the perfect TO solution, there are actually infinite other solutions for omnidirectional impedance matching. But in order to find these unusual solutions, one has to go beyond the local medium framework, where the original TO theory is rooted, into the much less explored realm of nonlocal photonic media. Such nonlocal media exist in neither natural materials like dielectrics, nor ordinary metamaterials which function as local effective media due to sub-wavelength structures.

Photonic crystals, with microstructures much larger than that of metamaterials, can also possess effective permittivity and permeability responses. When the excited bands and eigenmodes are near the Brillouin Zone center, photonic crystals can behave as effective media with zero refractive index [8]. While for bands and eigenmodes far away from the Brillouin Zone 
center, the effect of inherent nonlocality appears, which can turn photonic crystals into nonlocal photonic media with k-dependent parameters. To the surprise of most people, such nonlocality in photonic crystals can be utilized to realize of omnidirectional impedance matching [9].

\section{Advances in Science and Technology to Meet Challenges}

In Figs. 1(a) and 1(b), we show that the refractive behaviors of a photonic crystal slab with a "shifted" elliptical EFC are exactly the same as that of a metamaterial slab with an elliptical EFC at the Brillouin Zone center. It is astonishing that photonic crystals with such "shifted" EFCs could exhibit the rare property of omnidirectional impedance matching in TO media. It makes the photonic crystal nonreflective and totally transparent for incident light from any angle. This is clearly demonstrated in Fig. 1(c), where a transmittance $\mathrm{T}>99 \%$ for all angles within ($\left.89^{\circ}, 89^{\circ}\right)$ is achieved in a slab of the photonic crystal structure shown in the inset graph, irrespective of the number of layers. In Fig. 1(d), the nonlocal (kdependence) nature of the effective permittivity and permeability of this photonic crystal is clearly shown. The retrieved parameters (dots) excellently match with the derived theoretical requirement for omnidirectional impedance matching (dashed lines). In a certain sense, the omnidirectional impedance matching may also be understood as the expansion of the Brewster's angle from a single angle to all angles. Therefore, a type of "ultratransparent" nonlocal photonic medium beyond any dielectric is possible [9]. Such an ultratransparency effect also exist in one-dimensional photonic crystals [10]. Moreover, the effect could also be broadband and polarization-insensitive in some cases [10].

Since the ultratransparent photonic crystals exhibit controllable refractive behaviors and omnidirectional impedance matching, their functionality is very similar to the ideal TO media. In Fig. 2, we demonstrate an example of a wave concentrator. The original TO concentrator is discretized into four layers (shown in Figs. 2(a) and 2(b)) and a core. Each layer can be replaced by a corresponding type of ultratransparent photonic crystal with an EFC of the same shape, but "shifted" to the X point in k-space, as shown in Fig. 2(c). In the simulation results shown in Figs 2(d) and 2(e), one can see that the concentrating effects of the original TO and photonic crystal concentrators are almost the same, except for a phase difference of $\pi$ in the core region. Such a phase difference is induced by the shift of EFC in k-space. As shown in Fig. 1(b), an additional phase of $p d$ is added to the transmitted waves where $p$ denotes the shift of EFC in k-space and $d$ is the thickness of the photonic crystal. In other words, this phase difference is a signature of the nonlocality effect [9].

Ultratransparent photonic crystals can provide a pure dielectric platform for a large number of novel photonic devices such as nonlocal TO applications, wide-angle polarization filter, microwave transparent device, etc. One of the most important issues is the design strategy for the nonlocality in such photonic crystals. Besides the EFCs that control the direction of wave propagation, the effective surface impedance should be independently engineered, so as to ensure omnidirectional non-reflection. More optimization and engineering methods need to be further explored in order to find out the best strategy as well as the limit of

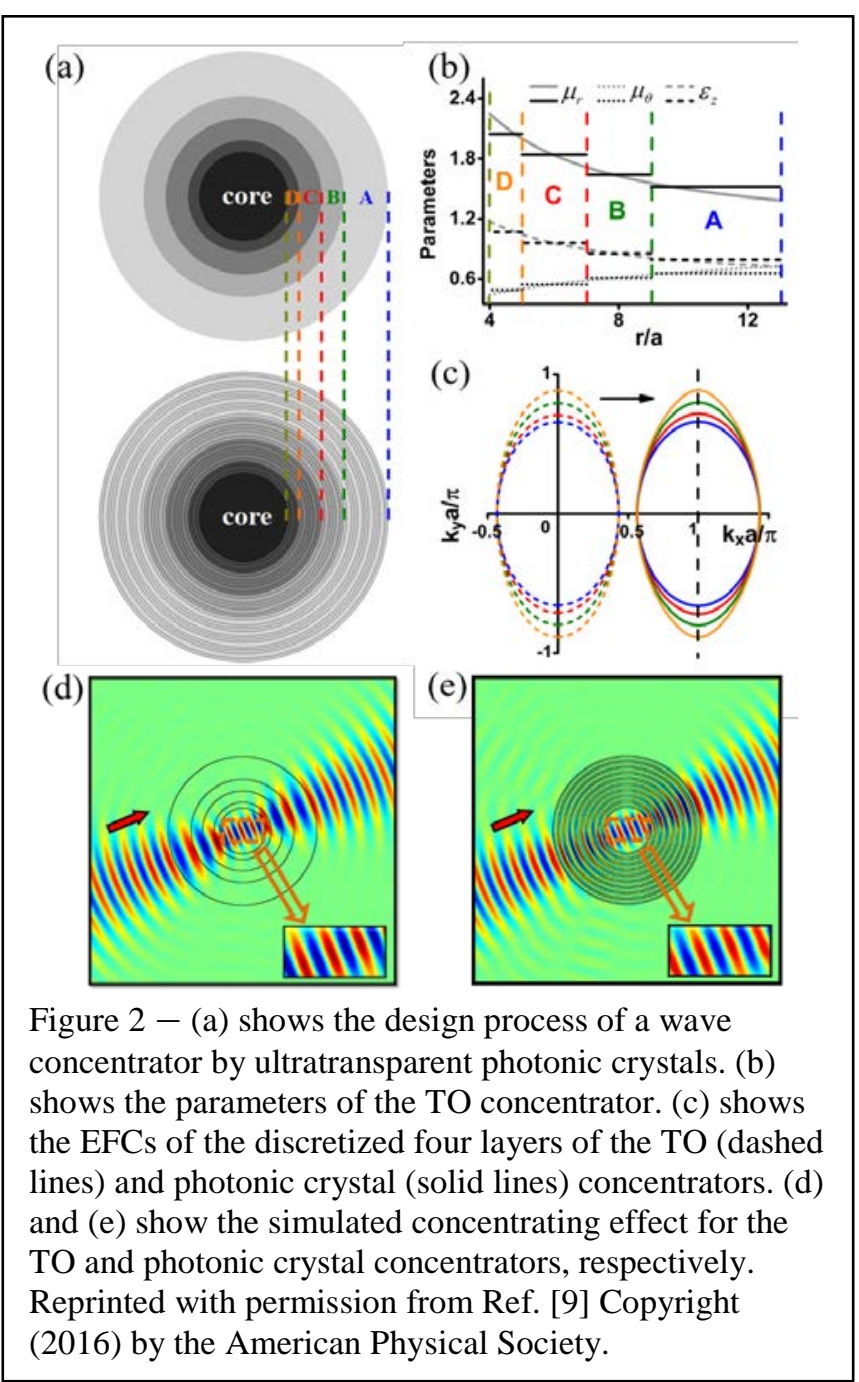

the realized nonlocal photonic media.

\section{Concluding Remarks}

The original TO theory was established in the field of metamaterials with local medium description. Now, pure dielectric photonic crystals with nonlocal effective parameters have shown an almost equivalent power in controlling both the refractive behavior and the impedance matching behavior, for the first time. 
With the advance in the fabrication techniques of large-scale photonic crystal with micrometer dielectric structures, this route may lead to experimental realization of ideal nonreflecting TO applications such as cloaking in the optical frequency regime.

Acknowledgments - This work has been supported by the State Key Program for Basic Research of China (No. 2014CB360505, No. 2012CB921501), National Natural Science Foundation of China (No. 11374224, No. 61671314) and a Project Funded by the Priority Academic Program Development of Jiangsu Higher Education Institutions (PAPD).

\section{References}

[1] Pendry JB, Schurig D, and Smith DR 2006 Controlling electromagnetic fields Science $\mathbf{3 1 2}$ 1780-1782

[2] Leonhardt U 2006 Optical conformal mapping, Science 312 1777-1780

[3] Schurig D, Mock JJ, Justice BJ, Cummer SA, Pendry JB, Starr AF, and Smith DR 2006 Metamaterial electromagnetic cloak at microwave frequencies, Science 314 977-980

[4] Lai Y, Ng J, Chen H, Han D, Xiao J, Zhang Z, and Chan CT 2009 Illusion optics: The optical transformation of an object into another object Phys Rev Lett 102253902

[5] Joannopoulos JD, Johnson SG, Winn JN, and Meade RD 2008 Photonic Crystals: Molding the Flow of Light (Princeton University Press, Princeton, USA, 2 ed.)

[6] Urzhumov YA, and Smith DR 2010 Transformation optics with photonic band gap media Phys Rev Lett 105163901

[7] Liang Z, and Li J 2011 Scaling two-dimensional photonic crystals for transformation optics Opt. Express 19 16821-16829

[8] Huang X, Lai Y, Hang ZH, Zheng H, and Chan CT 2011 Dirac cones induced by accidental degeneracy in photonic crystals and zero-refractive-index materials Nat Mater 10 582-586

[9] Luo J, Yang YT, Yao ZQ, Lu WX, Hou B, Hang ZH, Chan CT, and Lai Y 2016 Ultratransparent Media and Transformation Optics with Shifted Spatial Dispersions Phys Rev Lett 117223901

[10] Yao ZQ, Luo J, and Lai Y 2016 Photonic crystals with broadband, wide-angle and polarizationinsensitive transparency Opt Lett 41 5106-5109 


\section{Complexification in Transformation}

Optics -S. A. R. Horsley

University of Exeter

\section{Status}

Transformation optics is a theory where changing the coordinate system is used as a tool to solve the difficult problem of wave propagation in inhomogeneous materials. While we often take these coordinates as real numbers, transformation optics has even more power when we allow them to take complex values.

Undoubtedly, conformal transformations are the most widespread application of complex valued coordinates. They have long been applied to solve Laplace's equation in two dimensions, in areas such as electrostatics, fluid dynamics, and elasticity [1]. A conformal transformation takes the complex numbers $z=x+i y$ and $z^{*}=x-i y$ (formed from the Cartesian coordinates $x$ and $y$ ) to new complex numbers $w(z)$ and $w^{*}\left(z^{*}\right)$. Such a transformation leaves the form of Laplace's equation unchanged, and this form invariance allows one to map simple solutions in simple geometries, to equivalent solutions in more complicated ones. Meanwhile, a conformal transformation of the Helmholtz equation maps wave propagation in one refractive index profile, to propagation in another perhaps much more intricate one. Transformation optics has made heavy use of conformal transformations to design optical materials. For instance, Leonhardt [2] used a conformal transformation along with some clever analysis of a branch cut to design one of the first invisibility cloaks; and more recently Pendry and others [3] developed a theory of plasmonic energy concentrators based on conformal transformations.

But the utility of complex coordinates is much broader than conformal mapping. While a conformal map replaces the two-dimensional coordinates $x$ and $y$ with the single complex number $z$ and its complex conjugate $z^{*}$, one can also treat the $x, y$ coordinates in the wave equation as complex numbers themselves. Such a 'complexification' effectively doubles the number of dimensions of the space.

At the simplest level, this extension of the coordinate system allows one to find inhomogeneous materials that change the amplitude of a wave without generating any reflection (through a transformation like that shown in figure 1a). As a rough illustration consider a plane wave in $1 \mathrm{D}, \phi=\exp (\mathrm{inkx}$ ) (obeying the Helmholtz equation $\left.\mathrm{d}^{2} \phi / \mathrm{dx}^{2}+(n k)^{2} \phi=0\right)$. Performing a complex rotation of the $x$ coordinate $x=(a+i b) x^{\prime}$, we obtain either an exponentially growing or decaying wave, replacing the original real index $n$ with the complex index $(a+\mathrm{i} b) n$. As emphasized by Chew [4], the theory of the perfectly matched layer - nowadays the most common method for
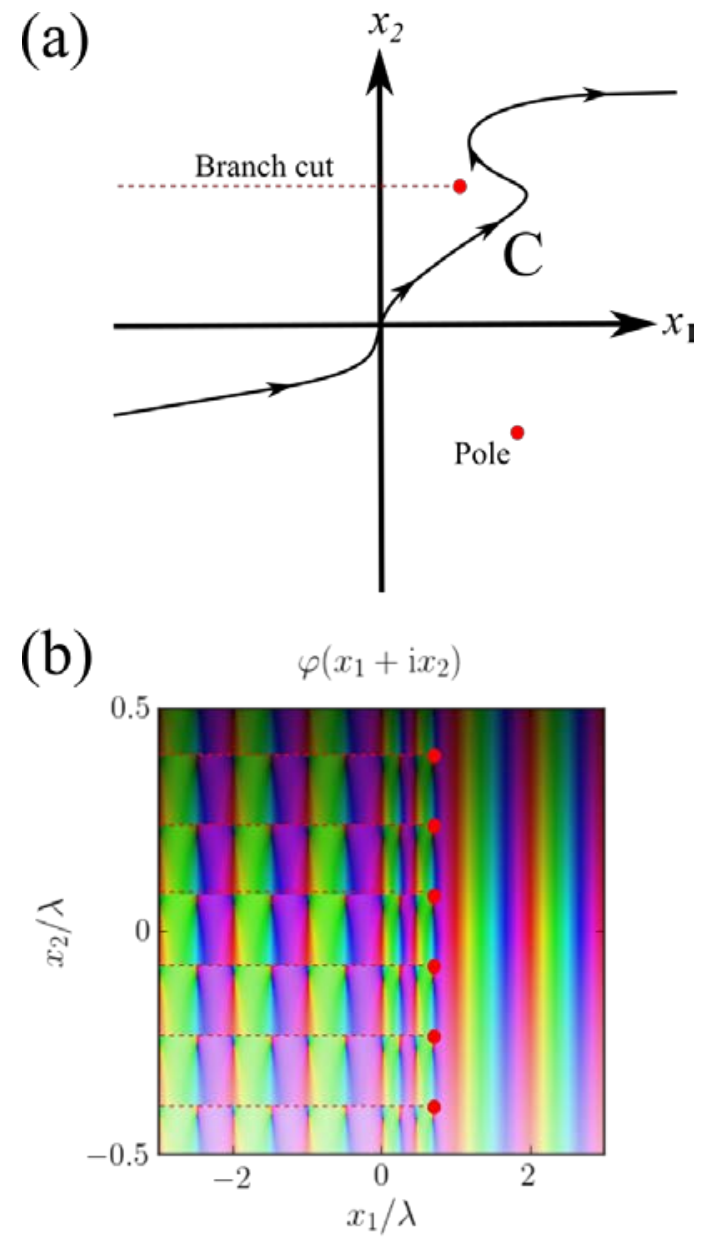

Figure 1: (a) Schematic of a complex coordinate transformation of the wave equation, where the real line $x=x_{1}$ is transformed to a curve $C$ that ventures into the analytically continued wave field. This process changes both the material parameters and the wave field in the wave equation; (b) an example of the numerical solution of the $1 \mathrm{D}$ wave equation in the complex plane, in this case showing a series of branch cuts (dashed red lines) emerging from poles along the imaginary axis.

truncating a computational domain - is founded on this approach.

Continued interest in transformation optics has led to new work in this area, applying complex valued spatial coordinates to design materials with inhomogeneous distributions of loss and gain [5-7]. This has been teamed with the simultaneous realisation that such 'lossy and gainy' optical materials can be used for the experimental investigation of a breed of complex valued ('PT symmetric') potentials that have long been a curiosity in quantum mechanics [8]. For example it has been found that reflectionless materials with $P T$ symmetry can be designed using a complex coordinate transformation of the Maxwell equations [6]. 
This 'complexified transformation optics' allows for an interesting way of thinking. When we ordinarily solve the wave equation in real space we don't consider the analytic continuation of the coordinates - the coordinates in the lab are real numbers! But, were we to look more carefully at the solution to the wave equation we would find particular features (e.g. poles or branch cuts) that occur in the wave field at complex values of the coordinates (see figure 1b). Through introducing an inhomogeneous anisotropic material, a complex coordinate transformation can move these features into real space $[5,6]$. Transformation optics with complex coordinates gives a physical meaning to the analytic continuation of a wave field!

Taking seriously the analytic continuation of the wave equation has recently provided some other new and rather general results [5,7]. These apply to planar media where the refractive index (or equivalently the permittivity) varies as a function of one of the coordinates $x$. If the refractive index $n(x)$ is an analytic function in either the upper or lower half complex $x$ plane then the material will not reflect waves incident from one side, whatever the angle of incidence. In addition, if $n(x)$ has only poles of order higher than 1 in the complex position plane then the slab will also have the same transmission coefficient as free space - it will be invisible from one side. The construction of such reflectionless media is in many cases also practically feasible, and one such material has recently been experimentally realised [9].

\section{Current and Future Challenges}

If we want to extend the reach of complex analysis in wave propagation problems there are both mathematical and practical challenges. Here I shall mention some which I know of, and some questions I think are interesting to ask.

I think the practical challenges are quite clear. Take the application of conformal transformations to cloaking, or to the design of plasmonic structures (e.g. gratings). Because conformal transformations are a special kind of coordinate transformation, then there is the question of what happens when we deviate from the condition of conformality. No experiment will be able to implement a refractive index profile accurate to an infinite number of decimal places. The same problem is also evident for the analytic profiles given in [7] - no experiment will be able to fabricate a profile that is exactly equivalent to a function that is analytic in the upper half complex position plane. There is always some tolerance to error, but the experiment needs to be within this tolerance. But In many cases I think it remains a challenge to build the devices we are inventing with transformation optics (whether using real or complex valued coordinates); both to develop a sufficient range of available material parameters, and to make the fabrication error small enough that the devices function as we intend them to.

Although there are many experiments in this area, I am just trying to make the point that many of our designs remain very difficult to put into practice are not yet practically feasible. This is especially true for some of the designs where both the real and imaginary parts of the material parameters are graded in space.

There are also some interesting mathematical questions to address. For instance, we know that making the coordinates complex can lead to general results concerning the reflection and transmission of waves through inhomogeneous media [5,7], and that features in the complex position plane can be given a physical meaning via transformation optics [6]. But to date we have largely based our understanding on the theory of functions of a single complex variable. Yet mathematicians have developed the theory of several complex variables - seldom used in optics - with concepts such as ramification taking the place of the branch cut. An interesting question is whether the theory of functions of several complex variables can be applied to derive useful information about the interaction of waves with structured media. In a similar spirit, it is also interesting to ask whether the theory of complex manifolds (and special cases such as the Kahler manifold) can be applied to complex coordinate transformation optics.

Another interesting connection that may be productive to explore, is the connection between complex coordinates and the one-way edge states that are currently attracting interest in the field of 'topological photonics' [10]. This connection does not seem to have been explored, but can be straightforwardly observed in the cases where the effective refractive index vanishes so that the field obeys the Laplace equation $\bar{\phi}=0$. Because the system only supports interface states that propagate in one direction, the field must become a function of a single complex variable e.g. only $z$, i.e. $\phi(z)=\exp (k z)=\exp [k(x+i y)]$.

\section{Advances in Science and Technology to Meet Challenges}

The above mathematical challenges pose questions that do not require any new mathematics, but require further time to answer. In contrast, I think the experimental realisation of many of the materials we design does require advances in technology.

Probably the most important is the ability to precisely and separately control the reactive and dissipative response of materials, so that refraction, loss and gain can all be separately graded in space. This could involve 
the development of metamaterial elements with a wide range of complex effective permittivity and permeability values. Gain is an especially difficult property to control, and present implementations often lead to instabilities.

Although there are cases where both real and imaginary parts of the material parameters have been separately controlled in space (see e.g. [9]), at the moment this is generally a difficult task. We should mention that [9] grades the resonant properties of metamaterial elements to obtain one of the non-reflecting index profiles derived in [7], using the frequency domain Kramers-Kronig relations to guarantee the required distribution of material properties. Such an approach may be useful more generally.

\section{Concluding Remarks}

Complex analysis is an old topic that is being given new practical relevance in the field of metamaterials research. It is used within transformation optics both through conformal transformations in two dimensions, and through the complex transformations and analytic continuation used to investigate materials with distributions of loss and gain. In the future there promise to be further applications of the mathematics of complex coordinates in optics, which may enable a deeper understanding of wave propagation in complex inhomogeneous media.

Acknowledgments - SARH acknowledges financial support from a Royal Society TATA University Research Fellowship (RPG-2016-186).

\section{References (separate from the two page limit)}

[1] Schinzinger R S and Laura P A A 1991 Conformal Mapping: Methods and Applications, Dover (New York)

[2] Leonhardt U 2006 Optical Conformal Mapping Science 312 1777-1780

[3] Pendry J B, Aubry A, Smith D R and Maier S A 2012 Transformation Optics and Subwavelength Control of Light Science 337 549-552

[4] Chew W C and Weedon W H 1994 A 3D perfectly matched medium from modified Maxwell's equations with stretched coordinates Micro. Opt. Tech. Lett. 7 599-604

[5] Horsley S A R, King C G and Philbin T G 2016 Wave propagation in complex coordinates J. Opt. 18044016

[6] Castaldi G, Savoia S, Galdi V, Alu A and Engheta N 2013 PT Metamaterials via Complex-Coordinate Transformation Optics Phys. Rev. Lett. 110173901

[7] Horsley S A R, Artoni M and La Rocca G C 2015 Spatial Kramers-Kronig relations and the reflection of waves Nat. Phot. 9 436-439
[8] Ruter C E, Makris K G, El-Ganainy R, Christodoulides D N, Segev M and Kip D 2010 Observation of parity-time symmetry in optics Nat. Phys. 6 192-195

[9] Jiang W, Ma Y, Yuan J, Yin G, Wu W and He S 2017 Deformable broadband metamaterial absorber engineered with an analytical spatial Kramers-Kronig permittivity profile Laser Phot. Rev. 111600253

[10] Lu L, Joannopoulos J D and Soljacic M 2014 Topological Photonics Nat. Phot.. 8 821-829 


\section{Surface Transformation Optics}

\subsection{Extending transformation optics and towards a}

thin cloak - Jensen Li, Jian Zhu, University of

Birmingham

\section{Status}

The interest of getting an invisibility cloak has a very long history but its main breakthrough came from the establishment of transformation optics (TO). It is the theoretical framework of TO to give us a recipe of the required material profile of a cloak while metamaterials provide us the necessary palette of material parameters. The first metamaterial cloak was realized in the microwave regime within half a year from the theory proposal [1]. Figure 1(a) shows the dispersion surface of a TO medium when a light bends from the freespace to the surface layer of a cloak while Fig. 2(a) shows the simulation of a cylindrical cloak for a plane wave coming from the left. The dispersion surface for both the TE and TM polarizations become generally elliptical with a change in shape to redirect light. The deformation gets bigger near the inner surface of cloak with more extreme material parameters. It was then quickly recognized that metamaterials cannot provide these extreme parameters for a cloak to work at optical frequencies due to the limit of resonance strength. One way out is to make a compromise on the functionality. If we conceal an object under a carpet instead of in the middle of air, the transformation involved compresses the object to a flat sheet instead of a point. This strategy makes the range of the required material parameters much less extreme. Cloaks for visible light, Fig. 2(b), become realizable (e.g. Ref [2]). Since then, we have been witnessing a dynamic period of development of TO in one of two directions: one on further simplifying cloaking strategies to have the ultimate application of cloak in the real world; another on extending $\mathrm{TO}$ as a framework to design optical components with functionalities beyond the conventional ones. The simplification route pushes the cloak towards higher frequencies, towards 3D applications, and towards cloaking objects of macroscopic sizes. The generalization route applies $\mathrm{TO}$ on getting devices beyond a cloak, e.g. to project an additional virtual object as an optical illusion, to design silicon photonic components with optimized performance, and to apply transformation approach to other kinds of waves.

A consideration on how to extend the fundamental principle of TO can actually benefit both directions. Coordinate transformation is at the heart of TO to get form-invariance of Maxwell's equations. A real 3-tuple $(\boldsymbol{x}, \boldsymbol{y}, \boldsymbol{z})$ is mapped to another 3-tuple. One possibility is to generalize the coordinates to complex numbers. It extends TO to include material gain and

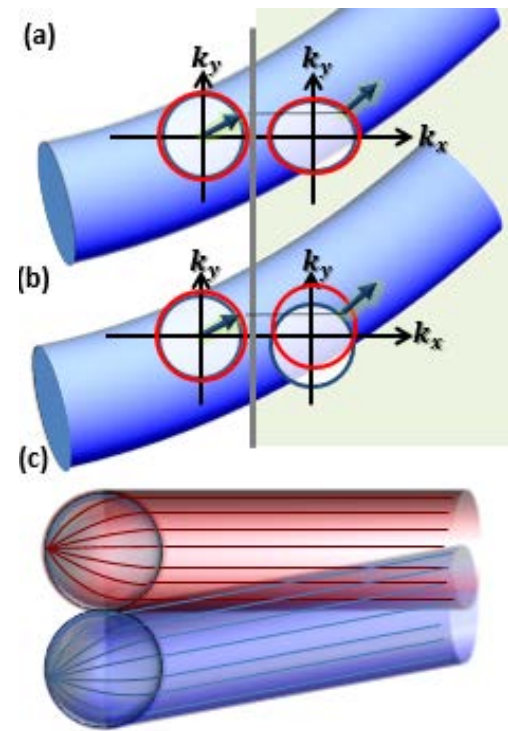

Figure 1 Ways to redirect light beam (a) by changing the shape of dispersion surface from TO-media, (b) by splitting dispersion surface from gauge transform-media. Vertical grey line indicates an interface. (c) Luneburg lens for getting variable directions from point sources.

loss so that active applications like fictitious source generation and one-way invisibility can be achieved [3]. On the other hand, we can add a fourth coordinate: time. A dynamic event cloak becomes realizable [4]. It turns out coordinate transformation is not the only possibility to get form-invariance. The transformation can also happen in the polarization, an internal degree of freedom, as a gauge transformation [5]. An example is an abstract rotation in the polarization space for $2 \mathrm{D}$ wave propagation:

$$
\left(\begin{array}{c}
E_{z}^{\prime} \\
i H_{z}^{\prime}
\end{array}\right)=\left(\begin{array}{cc}
\cos \phi(x, y) & -\sin \phi(x, y) \\
\sin \phi(x, y) & \cos \phi(x, y)
\end{array}\right)\left(\begin{array}{c}
E_{z} \\
i H_{z}
\end{array}\right) .
$$

The transformation induces a corresponding one on the material parameters and forms its own class of materials. Figure 1(b) shows the class generated from such an abstract rotation. The transformed permittivity has additional anisotropy $\boldsymbol{\epsilon}_{\boldsymbol{x} z}$ and $\boldsymbol{\epsilon}_{\boldsymbol{y z}}$. Equivalently, the dispersion is split into two polarizations by shifting the circular one into two opposite directions in the kspace (vs. deforming shape in Fig. 1(a)). This splitting can be interpreted as a gauge field (pseudo vector potential) for photon. It provides a "magnetic" force to redirect light and can be utilized to design a cloak [6]. Figure 2(c) shows the simulations for a cylindrical cloak designed with gauge field approach. The rays passing through the cloak are squeezed upwards (vs. Fig. 2(a)). This asymmetry occurs in opposite ways for the two polarizations. Such an asymmetry with the "magnetic" bending force gives us a route to design TO devices with asymmetric transmission functionality. Figure 2(d) shows an example to generate a one-way edge state, which propagates 
between two regions of metamaterials and is not reflected even when a sharp corner is encountered.

\section{Current and Future Challenges}

Although generalizations allow us to extend $\mathrm{TO}$ applications to new territories, a major current challenge on designing a cloak using the TO approach stays behind: to obtain a thin cloak, working for wide angles in 3D, which are essential for a wearable cloak. Taking the cylindrical cloak as example (Fig. 2(a) with ray-tracing), intuitively the outermost cylindrical layer is responsible for the light hitting the cloak at a shallow angle, while an inner one, together with the layers outside, are responsible for the light nearer to normal incidence. The angular operation thus comes from a finite thickness of the cloak. Unless very extreme material parameters are allowed, a small thickness and wide-angle operation are difficult to realize at the same time. It also means that we can go further on the simplification route to get a thin cloak but with limited viewing angle.

We have already seen some successful demonstrations along this direction. By going to the extreme case of only one single layer of metamaterial atoms, as a metasurface, a carpet cloak at the normal incidence can be designed by phase compensation to get a target reflected plane wave. Surprisingly, even though it is designed at normal incidence, the working angle can be as large as 30 degrees in a demonstrated 3D metasurface cloak [7] (Fig. 2(e)). For the carpet cloak, it is also desirable to go beyond a flat mirror to mimic an arbitrary background image on the floor.

\section{Advances in Science and Technology to Meet Challenges}
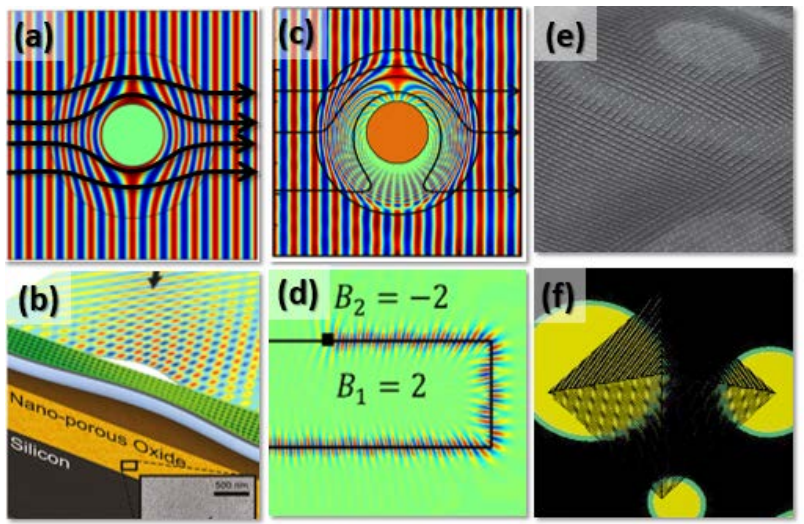

Figure 2 (a) and (b): cylindrical and carpet cloak at visible light from coordinate transform (from Ref.[2]). (c) and (d): gauge transform to get a cloak and one-way edge state. (e) and (f) are 3D carpet cloaks by metasurface (from Ref. [7]) and by lens array.

To enhance the working angle for a thin cloak, bearing in mind the issues we have discussed earlier, one natural step is to make a balance between working angles and thickness of cloak. Naturally, we can go from a single layer of metamaterial atoms to multiple layers or a thicker layer. Recently, the concept of Huygen's metasurfaces with two layers of metamaterial atoms have found excellent usage in enhancing transmission efficiency, utilizing both electric and magnetic response of the metamaterial atoms, with a homogenized boundary description of the metasurface [8]. Such a homogenized boundary description may be utilized to formulate TO instead of using effective medium parameters of the bulk. By designing successive layers of the metasurfaces, the cloaking behavior at a series of viewing angles can be obtained from normal to oblique incidence.

Another approach to get angular operation for a thin cloak takes advantage of the concept of integral imaging. Figure 1(c) shows the basic principle in redirecting light by a lens array. Unlike TO, in which dispersion surfaces are defined continuously at any locations inside the device, the device is pixelized into an array of lenses and each lens controls the direction of the generated light from a point source at different foci. Such an array can be used to project the desired 3D image you would like the observer to perceive in the case of a cloak. A preliminary demonstration by putting a 1D array of cylindrical lens on top of a digital display has achieved a cloak with viewing angle up to 13. $4^{\circ}$, varying in a single direction [9]. Although the viewing angle is limited due to paraxial geometrical optics, it gives a practical approach for a thin cloak with all three colors, with a compromise on spatial resolution of the image. To get an omnidirectional viewing angle, one can replace the $1 \mathrm{D}$ paraxial lens array by a 2D array of Luneburg lens (as Fig. 1(c) depicts). Figure 2(f) shows schematically if we assemble such Luneburg lens arrays into a pyramid, which projects the image on the floor consisting of circular-disk patterns to any angles. Furthermore, to focus light coming from different directions to the Luneburg lens array on a common plane, TO will be very favorable to realize flattened Luneburg lens [10] for the lens array.

\section{Concluding Remarks}

Based on the early works of TO together with metamaterials, we have now the basic language in designing a cloak. We believe there are still huge opportunities for us to develop TO. A fundamental approach in extending TO's theoretical framework should be beneficial to both getting a realistic cloak and designing optical components with properties beyond those given by conventional approaches. Probably, the wearable cloak for visible light will be an extension or combination of the TO technique with approaches such as metasurfaces and integral imaging. 
Acknowledgments - We acknowledge support from European Union's Seventh Framework Programme under Grant Agreement No. 630979.

\section{References (separate from the two page limit)}

[1] Schurig D, Mock J J, Justice B J, Cummer S A, Pendry J B, Starr A F, and Smith D R 2006 Metamaterial Electromagnetic Cloak at Microwave Frequencies Science 314 977-980

[2] Gharghi M, Gladden C, Zentgraf T, Liu Y, Yin X, Valentine J and Zhang X 2011 A carpet cloak for visible light Nano Lett. 11, 2825-2828

[3] Castaldi G, Savoia S, Galdi V, Alù $A$ and Engheta N 2013 PT Metamaterials via Complex-Coordinate Transformation optics. Phys. Rev. Lett. 110, 173901

[4] McCall M W, Favaro A, Kinsler P, and Boardman A 2010 A spacetime cloak, or a history editor $J$. Opt., 13, 024003

[5] Liu F and Li J 2015 Gauge field optics with anisotropic media Phys. Rev. Lett. 114103902

[6] Liu F, Horsley S A R and Li Jensen 2017 Invisibility cloaking using pseudomagnetic field for photon Phys. Rev. B 95075157

[7] Ni X et al 2015 An ultrathin invisibility skin cloak for visible light Science 349 1310-1314

[8] Pfeiffer C and Anthony G 2013 Metamaterial Huygens' surfaces: tailoring wave fronts with reflectionless sheets Phys. Rev. Lett. 19197401

[9] Choi J S and Howell J C 2016 Digital integral cloaking Optica 3536

[10] Hunt J, Tyler T, Dhar S, Tsai Y-J, Bowen P, Larouche S, Jokerst N M and Smith D R 2012 Planar, flattened Luneburg lens at infrared wavelengths Opt. Exp. 20 1706-1713 


\subsection{Combining curvature and index gradients for} surface and guided waves -

Rhiannon C Mitchell-Thomas ${ }^{1}$ and Oscar QuevedoTeruel $^{2}$

${ }^{1}$ University of Exeter

${ }^{2} \mathrm{KTH}$ Royal Institute of Technology

\section{Status}

Transformation optics has been proven to be a very versatile and powerful tool. It allows a link between geometry and the properties of a material to be formed, which is exact for waves propagating through a medium. The method employs a coordinate transformation to predefine the desired wave propagation characteristics, and this transformation is then used to calculate the appropriate permittivity and permeability with which to fill space so that this behaviour is achieved. For waves confined to a surface, geometrical optics provides a similar link between geometry and materials [1]. In contrast to the complex material properties required by transformation optics, the use of geometrical optics results in purely isotropic index profiles. These profiles are inhomogeneous, with a rotationally symmetric index gradient, meaning that they vary only along the radial direction of a polar coordinate system, but not the angular. It also allows for surfaces with non-zero curvature to be considered, which is not possible with transformation optics, and this provides new opportunities for device design.

Familiar lenses, such as the Luneburg and the Maxwell fish eye lens can be derived using geometrical optics. These isotropic, rotationally symmetric index profiles are accurate in the limit of geometrical optics, and hence the lenses must be electrically large to operate effectively. Using geometrical optics, equivalent surfaces for these types of lenses can be calculated [2]. For example, the Maxwell fish eye lens, which has a radially dependent refractive index profile that varies from one at the perimeter to two at the centre has an equivalent surface which is a homogeneous hemisphere. This lens has ray trajectories such that a point source positioned on the circumference will be imaged on the opposite side of the lens, due to the graded index. Equivalently, rays emitted from a point that are confined to the surface of a hemisphere would converge to a point on the opposite side. In this case, it is the curvature that accounts for the focusing, as the material of this surface is homogeneous. The equivalent surfaces for a whole family of these rotationally symmetric lenses have been derived, including those with singularities at their centres, and two examples of the latter are shown in figure 1 . However, there is a limitation. This is when the index profile increases at a rate equal to or more rapidly than $\mathbf{1} / r^{2}$. When this condition is reached, all rays that are

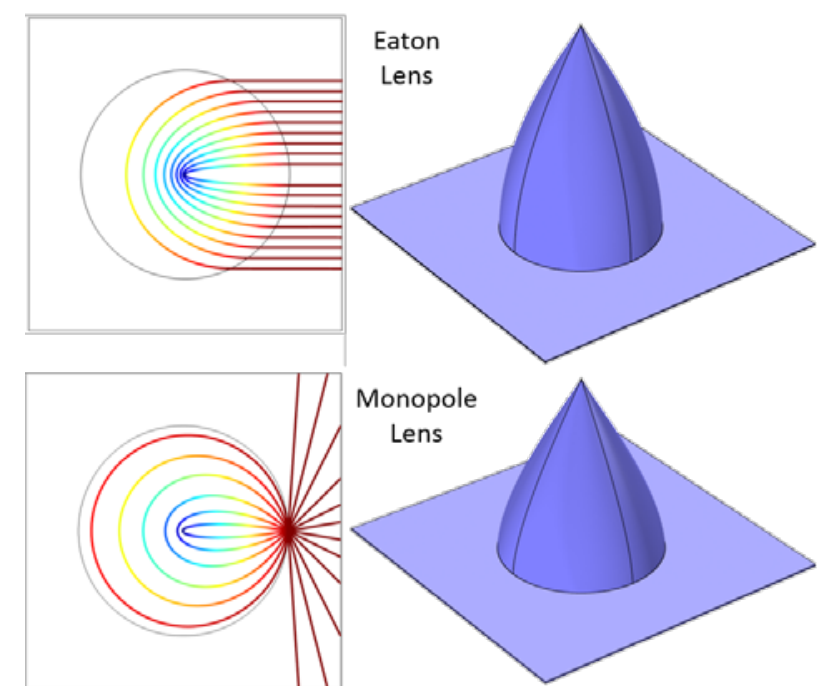

Figure 1 - Ray tracing in graded index profiles and the equivalent homogeneous surfaces for the Eaton lens and a Monopole lens.

incident on the profile are unable to escape and the equivalent surface is no longer closed.

These equivalent surfaces have found only limited practical application [3,4], and one of the reasons is that the shape of the curved surfaces may have undesirable features. For example, the surfaces shown in figure 1 are tall with respect to the radius, and also have a 90 degree transition when positioned on a flat surface. To overcome this issue, a combination of curvature and index gradients can be used to achieve the same behaviour, but with more freedom in the surface shape and index contrast can be limited to achievable values $[5,6,7]$. It is in this context that geometrical optics offers the most freedom in terms of practical device design, and full advantage can be taken of this versatile technique.

\section{Current and Future Challenges}

This combination of curvature and index gradients finds application in a number of areas, and three different devices will be discussed here. The first of which is a family of lenses that have very appealing properties, yet are impossible, or very difficult to fabricate in practice. Two examples are shown in figure 1, including the Eaton lens, which retro-reflects all rays that are incident on it, and a Monopole lens, which can be used so that an omnidirectional source only emits energy in one half-space of a 2D plane. However, the common feature of these lenses is that they exhibit a singularity in the centre of their refractive index profiles. It has been shown that curvature can be employed to emulate the influence of the singularity using a pointed tip at the centre, as shown in figure 1 , where the gradient of the tip is defined by the speed with which the profile approaches infinity [5]. In order to design a practical device, a graded index with a modest contrast of 1.6 is combined 
with a curved parallel plate waveguide so that the field is confined between the plates. The top image in figure 2 shows the electric field in this waveguide, and it can be seen to accurately retro-reflect the incident input beam so that the output beam exits the device in the same direction [5].

A further use of geometrical optics is to redesign lenses so that they are conformal to existing surfaces [6]. The increasing requirements for ever higher data transfer rates in wireless systems demands improved antennas with higher directivity, yet with decreased weight and volume that are less intrusive in the surrounding environment. One solution is to employ surface wave lenses, that are thin and lightweight, but with a large surface area. They can be applied to existing surfaces of vehicles, for example, but these surfaces may not be flat. In this case, it is known that the curvature of the surface will degrade the performance of a surface wave lens. However, it is possible to calculate a modified version of a graded index lens that is appropriate for a given surface with curvature. In the middle image of figure 2, a Luneburg lens on a curved surface is shown. It can be seen that the point source excitation is transformed to a plane wave output beam, which could then be leaked into a beam propagating in free space, creating the basis for a design of a conformal antenna [6].

Finally, an alternative version of a surface wave cloak can be created, without the use of the transformation optics technique [7]. In this case, a curved surface is employed to guide a wave up and over the object to be cloaked. This curvature will cause an undesired distortion to the incident wave and leave a signature. The route to eliminating any distortion is to counter the influence of the curvature with use of a graded index profile, so that this combination now has an equivalent surface that is flat and homogeneous. A surface cloak is shown in the bottom image in figure 2, where the input plane wave can be seen to be undistorted after propagating through a section of curved waveguide, due to the rotationally symmetric index which fills the curved region [7]. This type of cloak can be used to avoid scattering that would be caused by an obstruction on a surface.

\section{Advances in Science and Technology to Meet Challenges}

All of the above devices require an isotropic index gradient to be achieved, for which there are multiple approaches. Dielectric materials, engineered so that the relative permittivity can be precisely controlled are achievable with the combination of a low dielectric host medium loaded with high dielectric particles. In order to achieve a graded index, usually a discretization is applied and a number of layers are

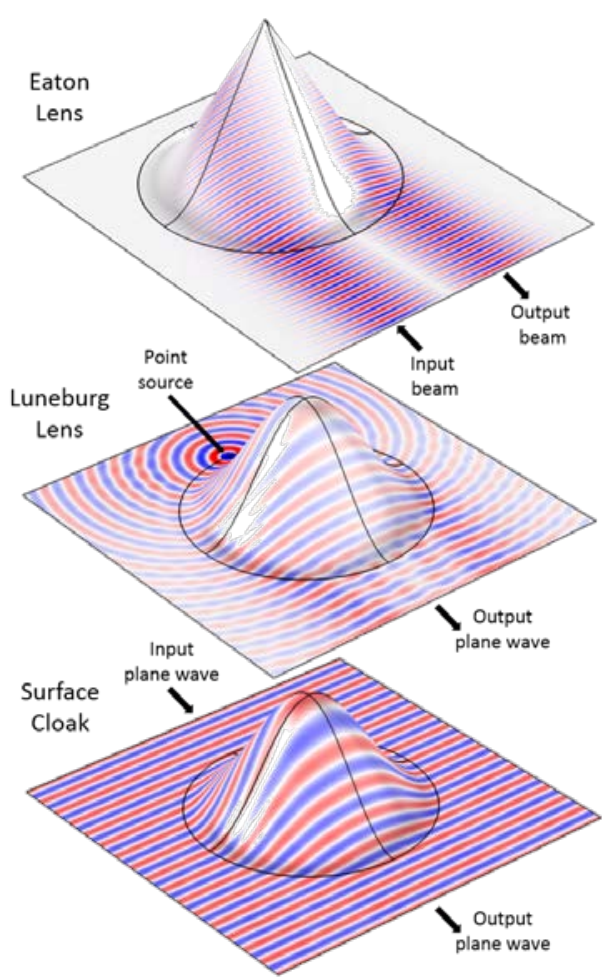

Figure 2 - Examples of devices designed using geometrical optics that employ curvature. Top: An Eaton lens with a modest index contrast of approx. 1.6. Middle: A Luneburg lens conformal to a curved surface. Bottom: A surface cloak where the influence of the curvature has been removed with an appropriate index profile.

individually cast [8]. For waves bound to an interface between a dielectric and free-space, it is also possible to achieve a change in speed of the supported mode simply by varying the thickness of a homogenous dielectric [9]. Alternatively, metasurfaces can be employed, where the index gradient is achieved by slowly varying the geometrical parameters of the individual meta-elements. This type of implementation would limit the operational bandwidth of a device due to the resonant nature of commonly used metasurfaces, but new advances in metasurface design have shown that these limitations can be considerably relaxed if higher symmetries are employed [10].

Rather than rely upon a rapid advance of a certain technology to meet requirements, this method of device design is chosen for its suitability with the current state of the art of manufacturing techniques. The fact that no complex anisotropy or simultaneous magnetic and electric properties are required is one of the major advantages of this technique.

\section{Concluding Remarks}

Here, a description of the application of geometrical optics to design a new range of devices has been given. This method allows curvature to be employed when creating surface or guided wave devices, which only 
require very basic material properties. The addition of this extra degree of freedom, surface curvature, permits the definition of purely isotropic materials and can negate the need for singularities in refractive index profiles. Three examples have been shown, including an Eaton lens with a finite and modest refractive index contrast, a conformal Luneburg lens, and a surface cloak, demonstrating the versatility of the technique. All of these devices can be fabricated by employing either metasurface or graded dielectric approaches.

Acknowledgments - The first author was funded by the Engineering and Physical Sciences Research Council (EPSRC), UK under Grand Challenges Grant (EP/N010493/1) "SYnthesizing 3D METAmaterials for RF, microwave and THz applications (SYMETA)."

\section{References}

[1] Leonhardt U and Philbin T G, 2010 Geometry and Light: The Science of Invisibility Dover, New York

[2] Sarbort M and Tyc T 2012 Spherical media and geodesic lenses in geometrical optics Journal of Optics 14075705

[3] Kunz K S 1954 Propagation of Microwaves between a Parallel Pair of Doubly Curved Conducting Surfaces Journal of Applied Physics 25642

[4] DuFort E C, Uyeda H A 1984 Geodesic DomeLens Antenna US Patent 4,488,156

[5] Horsley S A R, Hooper I R, Mitchell-Thomas R $\mathrm{C}$ and Quevedo-Teruel O 2014 Removing Singular Refractive Indices with Sculpted Surfaces Scientific Reports 44876

[6] Mitchell-Thomas R C, Quevedo-Teruel O, McManus T M, Horsley S A R and Hao Y 2014 Lenses on Curved Surfaces Optics Letters 39 3551-3554

[7] Mitchell-Thomas R C, McManus T M, QuevedoTeruel O, Horsley S A R and Hao 2013 Y Perfect Surface Wave Cloaks Physical Review Letters 111213901

[8] Quevedo-Teruel O, Tang W, Mitchell-Thomas R C, Dyke A, Dyke H, Zhang L, Haq S and Hao Y 2013 Transformation Optics for Antennas: Why limit the bandwidth with Metamaterials? Scientific Reports 31903

[9] Mitchell-Thomas R C, Quevedo-Teruel O, Sambles J R and Hibbins A P 2016 Omnidirectional surface wave cloak using an isotropic homogeneous dielectric coating Scientific Reports 630984

[10] Quevedo-Teruel O, Ebrahimpouri M, Ng Mou Kehn M 2016 Ultra Wide Band Metasurface Lenses Based on Off-Shifted Opposite Layers IEEE Antennas and Wireless Propagation Letters 15484 
5.3 Transformation optics at surfaces - Philippe Tassin and Vincent Ginis

Chalmers University of Technology and Vrije Universiteit Brussel

\section{Status}

About a decade ago, transformation optics established a new way to take advantage of the unprecedented properties of metamaterials for the design of optical devices. The formalism relies on the form-invariance of Maxwell's equations for the propagation of light in an inhomogeneous medium under coordinate transformations. This invariance leads to an equivalence relation between the components of a metric tensor and the permittivity and permeability of a medium. The design technique for optical devices then goes as follows: start from a Cartesian grid and bend the coordinate lines until they follow the desired path. In this way, one defines a metric tensor, which can subsequently be used to calculate the spatially varying permittivity and permeability functions needed for an optical device in which light rays follow paths along the transformed coordinate lines.

Transformation optics as a design technique for optical devices has three major advantages compared to traditional design techniques. First, it allows dividing the optical design into two steps. One starts from the desired electromagnetic field distributions to determine the material properties of the device. These material properties need then be implemented with nanostructured metamaterials - a challenging problem in itself. The second advantage is that transformation optics is, just as Gaussian optics for traditional optical instruments, a completely geometrical procedure. Finally, transformation optics is not diffraction-limited. Since it is based on Maxwell's equation, it is a fullwave technique, and optical devices designed by it do not suffer from geometrical aberrations.

\section{Current and Future Challenges}

In its original formulation from 2006, transformation optics is inherently a three-dimensional method. It relies on continuous transformations between coordinate systems in a three-dimensional space, generating permittivity and permeability functions that vary smoothly as a function of the space coordinates. This method creates optical devices with permittivity and permeability varying in all three dimensions.

However, this is not very desirable from a technological point of view. Metamaterials research has increasingly focused on the fabrication of so-called metasurfaces, two-dimensional arrays of meta-atoms, which are easier to fabricate and still allow for versatile transmission and reflection properties. Metasurfaces can now be designed to have almost any optical property in a flat or curved plane. This makes transformation-optical techniques applicable to metasurfaces very interesting.

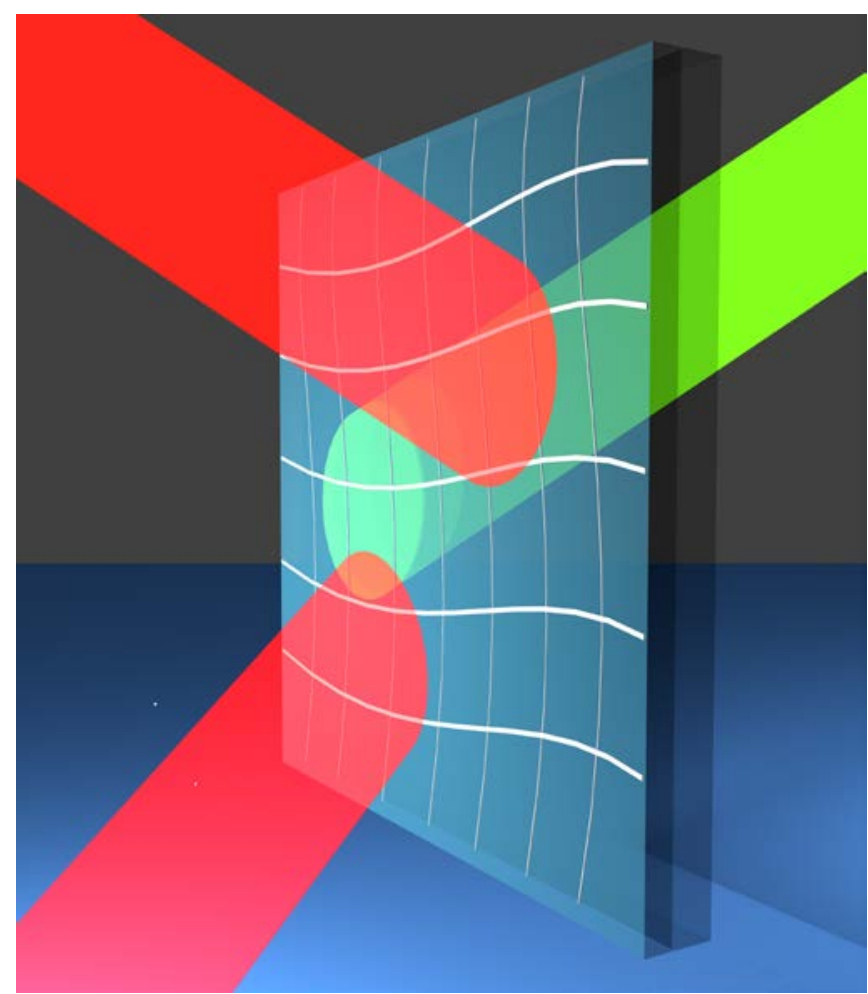

Figure 1 - Illustration of a light beam reflected and transmitted at the surface of a transformation-optical material. With the use of a coordinate transformation, we want to engineer the properties of the scattered beams-for example, direction of propagation, beam shape, and frequency.

When considering transformation optics at surfaces, we must distinguish between devices where a light beam is scattered by a transformation-optical surface (Figure 1) and devices where a light wave propagates along a transformation-optical surface (Figure 2).

In one of the first steps towards transformation optics on surfaces, a thin metamaterial coating was designed to enhance the optical force between two traditional optical waveguides [1]. If the metamaterial implements an annihilating transformation, then it is decreasing the effective interwaveguide distance experienced by the light waves in the underlying electromagnetic space, thus enhancing the optical forces between the waveguides. Annihilating transformations can be obtained from metasurfaces with a negative index of refraction.

Nevertheless, one can make use of discontinuous transformations to describe electromagnetic phenomena that occur at an interface between a common material (or air) and a metamaterial surface, e.g., the Goos-Hänchen shift [2]. If the metamaterial is the transformation-optical equivalent of a coordinate transformation, then discontinuous transformations 
allow us to understand and engineer phenomena at the surface of nanophotonic structures in terms of a geometrical framework.

A second reason for wanting to apply transformation optics to thin two-dimensional optical systems is that optical devices are increasingly being integrated on photonic integrated circuits. One of the main advantages of integration is of course a smaller footprint. However, with routing of optical signals in such circuits being achieved with strip or rib waveguides, bends and processing elements become less efficient with further miniaturization. A full-wave design technique such as transformation optics could thus advance the miniaturization of integrated optical circuits.

A number of research groups have recently made progress in designing two-dimensional transformationoptical devices for in-plane guided waves. By placing a metamaterial on top of a metal film, it is possible to steer surface plasmon waves along a bend, focus them, or make them pass over an obstacle [3,4]. These approaches add a metamaterial on top of a nanophotonic waveguide. By locally modifying the Fermi level of graphene, it was also proposed to create a two-dimensional transformational-optical surface that can split or focus surface waves on graphene. Here, the guided-wave-supporting surface is modified by a transformation [5]. In a dielectric waveguide, it is also possible to locally adapt the thickness to achieve a desired spatial distribution of mode indices implementing a transformation optics design [6] at the expense of backscattering.

Recently, an alternative transformation optics theory was developed for guided waves on integrated optical waveguides. Indeed, one problem with transforming integrated optical waveguides is that traditional transformations optics imposes metamaterials in both the core and the cladding of the waveguide. This results in bulky structures that are difficult to fabricate. However, if one also varies the thickness of the waveguide, it is possible to transform guided waves in waveguides where the core is made of a metamaterial, but the cladding is left unaltered $[7,8]$. The thickness variation is engineered such that the dispersion relation is unaltered before and after the transformation. With this method, beam splitters and beam benders have been designed.

\section{Advances in Science and Technology to Meet Challenges}

The further development of surface transformation optics at surfaces will require innovations in theory as well as in nanofabrication. For the modification of light beams incident on a surface, as visualized in Figure 1, transformation optics has been shown to be applicable to homogeneous surfaces, but there is no geometrical theory for inhomogeneous surfaces. Inhomogeneous metasurfaces were demonstrated to be able to achieve advanced beam manipulation, e.g., generalized refraction [9], but it is currently not completely understood how to describe and engineer this response using coordinate transformations.

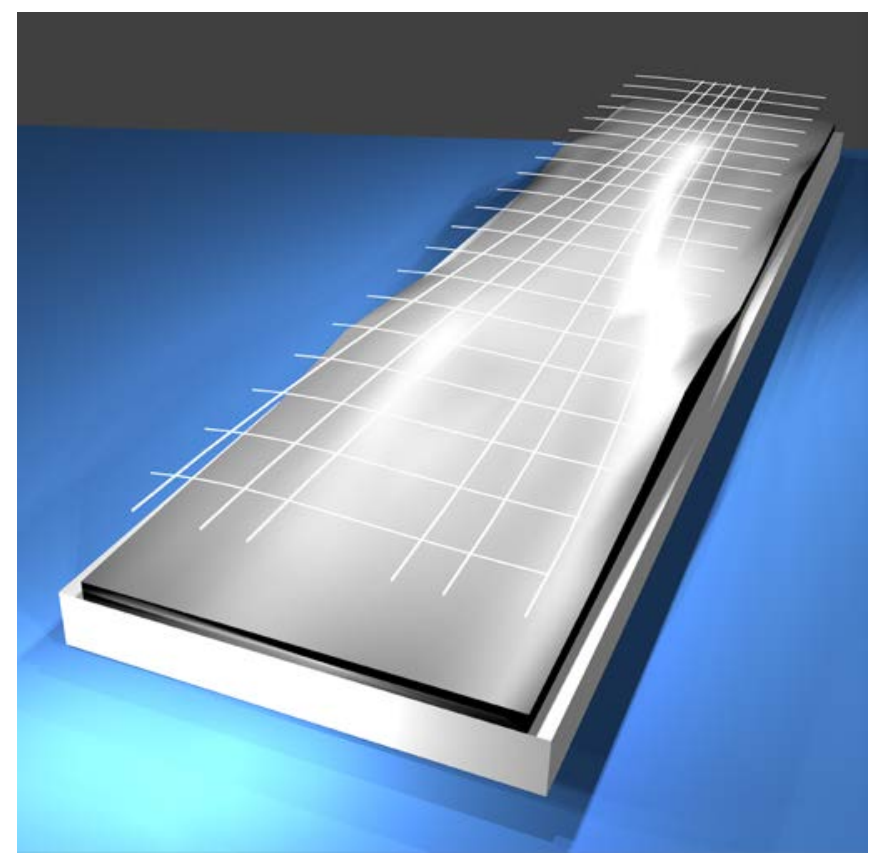

Figure 2 - Illustration of transformation optics in lightwave technology. With the use of transformation optics implemented with inhomogeneous metasurfaces or waveguides, photonic functions such as steering, wave reshaping, splitting and recombining can be achieved.

For the design of guided wave devices, as visualized in Figure 2, the theory of transformation optics is now well understood [6-8], but advances in nanofabrication are clearly required. Surface transformation-optical designs require an anisotropic dielectric material layer with varying height. This may be achieved with direct laser writing, similarly to how an optical ground plane cloak was fabricated by creating a matrix of spherical particles of varying size [10]. By replacing the spherical particles with ellipsoidal particles, it is possible to get anisotropic optical properties. At the same time, one can investigate how the complex parameters can be further reduced to meet the current state-of-the-art in fabrication.

\section{Concluding Remarks}

In the past decade, transformation optics has revolutionized our understanding of and capabilities to design the interaction between light and threedimensional nanostructured devices. However, for 
many technological applications it is even more important to be able to understand and design the interaction between light and two-dimensional media. This would allow for the manipulation of free-space travelling beams as well as for in-plane guided waves. In our opinion, further advances in this field, both theoretical and fabricational, are needed to accelerate the widespread use of transformation optics as a geometrical tool to design next-generation optical devices.

Acknowledgments - The authors acknowledge funding from the Area of Advance Nanoscience and Nanotechnology, the Research Foundation of Flanders (Fellowship No. 12O9115N), and COST (Action MP1403 on Nanoscale Quantum Optics).

\section{References}

[1] Ginis V, Tassin P, Soukoulis CM and Veretennicoff I 2013 Enhancing optical gradient forces with metamaterials, Phys. Rev. Lett. 110, 057401

[2] Lambrechts L, Ginis V, Danckaert J and Tassin P 2017 Transformation optics for surface phenomena: Engineering the Goos-Hänchen effect, Phys Rev B 95, 035427

[3] Huidobro PA, Nesterov M, Martin-Moreno L and Garcia-Vidal FJ 2010 Transformation Optics for Plasmonics, Nano Lett 10, 1985-1990

[4] Liu Y, Zentgraf T, Bartal G and Zhang X 2010 Transformational Plasmon Optics, Nano Lett 10, 1991-1997

[5] Vakil A and Engheta N, Transformation Optics Using Graphene 2011 Science 332, 1291-1294.

[6] Gabrielli LH, David L, Johnson SG and Lipson M 2012 On-chip transformation optics for multimode waveguide bends, Nature Commun 3, 1217

[7] Viaene S, Ginis V, Danckaert J and Tassin P 2016 Transforming two-dimensional guided light using nonmagnetic metamaterial waveguides, Phys $\operatorname{Rev} B$ 93, 085429

[8] Viaene S, Ginis V, Danckaert J and Tassin P, Mitigating optical singularities in coordinate-based metamaterial waveguides 2017 Phys Rev B 95, 155412

[9] Yu N, Genevet P, Kats MA, Aieta F, Tetienne J-P, Capasso F and Gaburro Z 2011 Light Propagation with Phase Discontinuities: Generalized Laws of Reflection and Refraction, Science 334, 333-337

[10] Ergin T, Stenger N, Brenner P, Pendry JB and Wegener M 2010 Science 328, 337-339 (2010). 


\section{Antennas}

\subsection{Metasurface Radiation and Guidance at} Microwaves- Enrica Martini, Gabriele Minatti, Stefano Maci, University of Siena

\section{Status}

Controlling the electromagnetic (EM) field has become a popular research topic in the recent years thanks to the advances done in the framework of metamaterials (MTMs). Metasurfaces (MTSs), i.e. the twodimensional equivalent of MTMs, have gained a large interest due to their engineering applicability to a vast range of frequencies. MTSs are constituted by arrangements of electrically small metallic/dielectric inclusions in a regular planar lattice inside or on top of a thin dielectric layer, which can be manufactured with standard PCB technologies or additive manufacturing. While MTMs characterization is given in terms of equivalent volumetric constitutive parameters, MTSs characterization is given in terms of homogenized boundary conditions (BCs) defined in terms of a spacevariable surface impedance. Through a proper spatial modulation of these BCs one can control surface/plasmonic wave propagation and radiation in a simpler way than volumetric waves in MTMs. The BCs are modified by spatially changing the characteristics of the small inclusions in a gradual manner, while maintaining uniform the periodic lattice.

Depending on the phenomenon we would like to control, we can identify three classes of MTSs:

(i) phase-gradient MTSs for space-wave control;

(ii) phase-gradient MTSs for surface-wave (SW) control;

(iii) sinusoidally-modulated MTSs for radiation control.

Phase gradient MTSs for space wave control ([1]) and their generalization called "Huygens MTSs" ([2], [3]), are designed with the objective to transform the wavefront and polarization of space-wave fields. A general wavefront can be obtained by locally controlling the gradient of the phase of the local transmission coefficient. Thin lenses, beam deflectors, beam splitters fall in this class. From an engineering point of view, these MTSs can be seen as a generalization of transmitarrays to continuous boundaries, while from a physical point of view, they implement the equivalence theorem.

Phase gradient MTSs for SW control have the objective of controlling surface/plasmonic wave propagation path and wavefront. These MTSs mold the wavefront during the propagation, similarly to what happens in transformation optics 3D media, but maintaining the wave bounded. The phenomenon is sometimes called "Metasurfing" [4]. Planar lenses, cloaking devices, and general beam forming networks without transmission lines belong to this category. Their behavior can be described through an analogous to geometrical optics in equivalent graded index materials or through a planar version of Transformation Optics [5].

Sinusoidally-modulated MTSs have the objective to locally change the dispersion properties of a bounded SW so as to transform it into a leaky wave. These MTSs can be directly used as antennas by exciting them with a coplanar feeder consisting of an elementary radiator. For centered-point fed MTSs, the impedance $\mathrm{BCs}$ are modulated with quasi-periodic functions in the radial direction, thus transforming the cylindrical SW excited by the feeder into a desired radiating aperture-field distribution. The feasibility of the concept was first demonstrated in [6] and [7] and more recently systematized in [8]. The appeal of these MTS antennas is due to the capability to obtain a complete control of the aperture field distribution in terms of amplitude, phase and polarization through an almost analytic design procedure [8]. The possibility of achieving also amplitude control renders these antennas much more flexible than reflectarrays; furthermore, a low profile is achieved since the feed is in-plane and not external to the surface. Finally, low losses are obtained thanks to the absence of a beam forming network and to the non-resonant nature of the MTS inclusions.

\section{Current and Future Challenges}

MTSs have been proven to be an exceptionally effective tool for controlling EM waves. However, several challenges are still open and need to be addressed for broadening application fields or improving the performances.

Concerning type (i), a full tailoring of any space field, including reactive field, is still an issue, due to the dependence of the MTS phase discontinuity on the incidence direction and due to the limited bandwidth. A relevant challenge consists on MTSs that can be used to emulate differential or convolution operators, investigation pioneered in [9]. A further issue is the one of non-reciprocal MTSs (both gyrotropic and nongyrotropic). Achieving non-reciprocity requires breaking the time reversal symmetry using an external force, like ferromagnetic materials; however, this can be obtained also by using active unidirectional elements in an active MTS layer configuration. This opens new interesting applications like one-way screens, isolating radomes, radar absorbers and thin cloaks [10]. Several groups have also progressed in parity-type symmetric non-local MTSs, with the objective to construct super focusing lenses [11]. 
Concerning phase gradient MTSs for surface and plasmonic waves (type (ii)), these are still poorly explored in the microwave regime. The extension to non-regular grids and to time domain could be among the most important challenges. We can conceive structures able to change the shape of an impulse during the propagation over the surface. Hyperbolic planar surfaces may achieve focusing properties similar to those of hyperbolic media, but in-plane. These typologies include graphene and new low dimensional materials [12].

Concerning sinusoidally-modulated MTSs for radiation control (type (iii)), the most relevant current challenge concerns the increase of the operational bandwidth, especially when the antenna pattern is associated with shaping or dual polarization capability. The bandwidth problem is related to the dispersive nature of the solutions currently adopted to implement the artificial BCs and may actually limit the applicative fields. Multilayer MTSs have not been exploited yet in this field and may lead to significant benefits in terms of bandwidth. Polarization independent surfaces (and therefore dual polarization antennas) is another current research challenge. Some work has already been done in this direction; a polarization independent MTS antenna has been designed in a narrow bandwidth [13]. In [14], polarization insensitive MTSs are proposed, which work in a wide band, but the absence of a ground plane reduces the efficiency in their usage as antennas.

Future challenges are concerned with the generalization and refinement of modelling tools. Indeed, as a MTS-based device usually involves several thousands of unit cells, possibly containing some small geometrical details, a full wave analysis of the whole structure is not feasible with general-purpose software tools. It is therefore necessary to develop effective, ad hoc analysis and design tools. Furthermore, additional efforts are required to make the modelling tools faster, for the purpose of embedding them into proper optimization procedures. Also, incorporating electronic devices or tunable materials in the MTSs would require an update of the modelling tools.

\section{Advances in Science and Technology to Meet Challenges}

Among the future challenges in the microwave regime for the typology (iii) - also sometimes common to (i) and (ii) - the most relevant one is probably concerned with the design of dynamically adaptive and reconfigurable MTSs, namely surfaces that can change their configuration subjected to an external programmable control, or ultimately, energized by the incoming wave. Associating an active component to each small inclusion of the MTS would allow for a local control of the BCs, leading to the possibility of dynamically configuring the MTS behavior. Hyper density of elements and complex biasing networks are the major challenges. Micro electromechanical devices (MEMS) seem to be inappropriate for this scope due to the relevant problems of reliability. On the other hands, tunable materials like liquid crystals, being developed for optical scopes, suffer at microwave frequencies of significant losses and slow commutation times for certain applications. Among the phase changing materials, the vanadium dioxide (VO2), despite its interesting properties of exhibiting thermal phase transition that can be exploited by optical pumping, is difficult to be controlled in the operational environment, and it is still at a low technological readiness levels. Optical pumping associated with MTSs printed on silicon or gallium arsenide (GaAs) is instead presently more mature.

Another interesting development from both modeling and realizations is the one towards conformal MTSs, appealing for instance for devices on board of vehicles or aircrafts.

Concerning non-reconfigurable MTSs, the main technological challenges are related to their fabrication. One the one hand, high precision processes are needed to move towards higher frequencies. On the other hand, the fast development of 3D printing technologies opens a range of opportunities for the realization of new, low cost structures.

Finally, advances in science and technology are mainly related to the implementation of dynamically adaptive MTSs. Technological solutions that have been proposed up to now have indeed severe limitations. Tunable materials suffer of high losses, low speed, large temperature sensitivity or small tunability range. Active standard components are characterized by nonnegligible losses and high complexity of the bias network. Electromechanically devices have low reliability.

\section{Concluding Remarks}

Encompassing with imagination, the future MTS could be interpreted as a conformal surface whose characteristics can be adapted time-to-time to the needs, changing the role of the surface or of its subparts from guiding structures to radiative devices. The research framework on MTSs is wide, still in progress, and potentially not limited to the ICT world.

\section{References}

[1] N. Yu et al., "Flat Optics: Controlling Wavefronts with Optical Antenna Metasurfaces," in EEE Journal of Selected Topics in Quantum Electronics, 
vol. 19, no. 3, pp. 4700423-4700423, May-June 2013.

[2] C. Pfeiffer, A. Grbic, "Metamaterial Huygens' Surfaces: Tailoring Wave Fronts with Reflectionless Sheets," Phys. Rev. Lett., vol. 110, no. 19, pp. 197401, May 2013.

[3] A. Epstein and G. V. Eleftheriades, "Passive Lossless Huygens Metasurfaces for Conversion of Arbitrary Source Field to Directive Radiation," in IEEE Transactions on Antennas and Propagation, vol. 62, no. 11, pp. 5680-5695, Nov. 2014.

[4] S. Maci, G. Minatti, M. Casaletti and M. Bosiljevac, "Metasurfing: Addressing Waves on Impenetrable Metasurfaces," in IEEE Antennas and Wireless Propagation Letters, vol. 10, no., pp. 1499-1502, 2011.

[5] E. Martini, M. Mencagli, D. González-Ovejero and S. Maci, "Flat Optics for Surface Waves," in IEEE Transactions on Antennas and Propagation, vol. 64, no. 1, pp. 155-166, Jan. 2016.

[6] B. H. Fong, J. S. Colburn, J. J. Ottusch, J. L. Visher and D. F. Sievenpiper, "Scalar and Tensor Holographic Artificial Impedance Surfaces," in IEEE Transactions on Antennas and Propagation, vol. 58, no. 10, pp. 3212-3221, Oct. 2010.

[7] G. Minatti, F. Caminita, M. Casaletti and S. Maci, "Spiral Leaky-Wave Antennas Based on Modulated Surface Impedance," in IEEE Transactions on Antennas and Propagation, vol. 59, no. 12, pp. 4436-4444, Dec. 2011.

[8] G. Minatti et al., "Modulated Metasurface Antennas for Space: Synthesis, Analysis and Realizations," in IEEE Transactions on Antennas and Propagation, vol. 63, no. 4, pp. 1288-1300, April 2015.

[9] A. Silva, F. Monticone, G. Castaldi, V. Galdi, A. Alù and N. Engheta, "Performing Mathematical Operations with Metamaterials,” Science, vol. 343, no. 6167, pp. pp. 160-163, 2014.

[10] S. Taravati; B. A. Khan; S. Gupta; K. Achouri; C. Caloz, "Nonreciprocal Nongyrotropic Magnetless Metasurface," in IEEE Transactions on Antennas and Propagation, doi: 10.1109/TAP.2017.2702712.

[11] F. Monticone, C. Valagiannopoulos, A, Alù, "Parity-Time Symmetric Nonlocal Metasurfaces: All-Angle Negative Refraction and Volumetric Imaging”, Phys. Rev. X., Vol. 6 no. 4, pp. 4101841031, Oct. 2016.

[12] A. Vakil and N. Engheta, "Transformation optics using graphene,” Science, vol. 332, no. 6035, pp. 1291-1294, 2011.

[13] A. Tellechea Pereda et al., "Dual Circularly Polarized Broadside Beam Metasurface Antenna," in IEEE Transactions on Antennas and Propagation, vol. 64, no. 7, pp. 2944-2953, July 2016.
[14] M. Li, S. Xiao, J. Long and D. F. Sievenpiper, "Surface Waveguides Supporting Both TM Mode and TE Mode With the Same Phase Velocity," in IEEE Transactions on Antennas and Propagation, vol. 64, no. 9, pp. 3811-3819, Sept. 2016. 
6.2 Bespoke lenses: An opportunity for tailoring antenna radiation patterns - Oscar Quevedo-Teruel, Mahsa Ebrahimpouri

KTH Royal Institute of Technology

\section{Status}

The concept of transformation optics was defined in 2006 [1]. Since then, the electromagnetic engineering community has been working on how to use this concept to improve the conventional properties of microwave circuits and antennas [2]. However, engineers found a major limitation in the first implementations based on analytical transformations: narrow bandwidth of operation. This narrow band is related to the need for highly anisotropic materials with permittivity and permeability components lower than the unity [3].

To overcome this initial limitation, two implementations were proposed: Non-Euclidean [4,5] and quasi-conformal transformations [6-8]. Both techniques demonstrated that fully dielectric and isotropic materials are possible for the design of optically transformed lenses. The non-Euclidean technique does not assume any approximation, but it is limited to two-dimensional designs. On the other hand, the quasi-conformal technique assumes approximations, but it can be employed to produce three-dimensional lenses. If the employed materials have low losses, these lenses can be used to produce a new kind of broadband directive antennas [7].

However, here again, there was a theoretical limitation. The existing implementations of quasi-conformal transformations did not take into account the properties of the feeding antenna. This difficulty was not noticed when an original lens, for example a Luneburg lens, was re-shaped into a different coordinate system [6]. However, when the transformation was employed to directly producing a lens [8], the technique was limited only to omnidirectional feeding sources.

Recently, the original theory of quasi-conformal transformations has been extended to produce lenses ad-hoc to a given feeding antenna [9]. This is the concept of 'bespoke lenses'. Let's assume a specific feeding, for example, a dipole, a monopole, or a microstrip patch antenna. All these sources are not omnidirectional, and they have certain radiation properties, including a given directivity. These radiation properties are mainly given by the far-field phase distribution of the electric and magnetic fields. For example, on the left side of Fig. 1, we have included two possible cases of phase distribution. These phase distributions will produce a certain farfield radiation pattern that can be derived analytically or numerically.

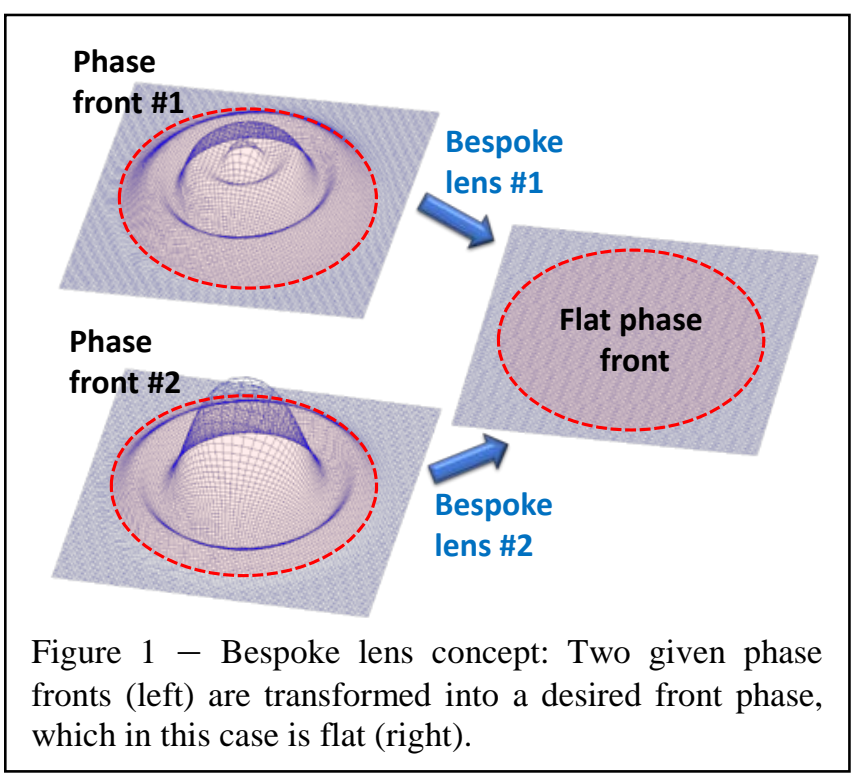

One common requirement for an antenna is to produce a planar front phase in a determined direction, which is a flat phase front as represented in Fig. 2, right side. A bespoke lens will be the quasi-conformal transformed lens that can match the original front phase to the desired pattern, in this case, a flat one. However, this theory is not limited exclusively to achieve a plane wave. It can be extended to other desired radiation patterns. For example, the radiation pattern may have a broader beam, or multiple beams in given directions. In that case, the required phase will not be a flat surface, but the required front phase for obtaining the desired radiation pattern.

One of the advantages of this technique is that the transformation will only require isotropic materials, since it is quasi-conformal. Additionally, if we assume a slight degradation of the results, simply removing the regions in which the refractive indexes are smaller than unity [6], the lens can be implemented with fully dielectric materials. If these dielectric materials are carefully chosen, our antenna can have inherently low losses, and an ultra wide band response [7].

In order to illustrate the potential of this technique, in Fig. 2, we show the results of a bespoke lens design. This figure illustrates the phase front in three orthogonal planes after applying the 'bespoke' concept to a holey spiral feeding antenna. More details about this implementation can be found in [9]. These results demonstrate that even in the case of a circular polarized antenna, it is possible to achieve a constant phase front for a given feeding. In terms of radiation pattern, the bespoke lens has contributed to increase significantly the directivity of a given spiral at the broadside direction. These results are independent of the frequency, as far as the dielectric constant of the employed materials are stable with frequency, and the lens size is large enough to produce an increase of the aperture of the antenna. 


\section{Current and Future Challenges}

As we have previously mentioned, the concept of bespoke lenses has been very recently introduced in [9]. However, it has been only demonstrated through simulations. In [9], bespoke lenses were designed for an aperture feeding, a spiral with circular polarization, and an ultra wide band slot. In all these cases, simulated results demonstrated the advantages of using this approach.

Although the benefits of bespoke lenses are clear, the manufacturing complexity is increased with respect to conventional lenses. In order to implement these lenses, a discretization is needed. The discretization level will depend on the complexity of the changes required for the desired phase. Then, a number of low loss dielectric materials must be synthesized. Finally, these materials must be drilled or molded to obtain the designed shapes. Although these shapes are commonly rounded, their cost of manufacturing is typically higher than a conventional hemi-spherical or elliptical lens.

Therefore, the challenge is to produce dielectric materials with low losses that could be synthesized at low cost with relative freedom in terms of molded shapes.

\section{Advances in Science and Technology to Meet Challenges}

A few years ago, the scientific opinion was that lens antennas were reminiscent of the 80s, which were already proven to be unsuccessful. Most of the researchers in the field of antennas accepted that arrays and reflectors were the only two techniques that could be employed to produce efficient and directive antennas. However, expert opinion has drastically changed in the last few years.

Most of the new wireless applications are expected to be allocated at higher frequency bands. At those frequencies, arrays are expensive due to the cost of their feeding networks. Additionally, the losses of these networks are extremely high, even when the structures are fully metallic. On the other hand, reflectors can only produce steerable angles with mechanical engines. In this situation, lenses started to be considered as a suitable technique to produce low cost and highly directive antennas at high frequencies.

Additionally, two other technological advances have contributed to increasing the popularity of lens antennas. The first one is the growing development of metasurfaces. Metasurfaces can be employed to achieve equivalent refractive indexes and, therefore, to implement two-dimensional lens antennas. Recent discoveries have demonstrated that when these metasurfaces are generated with higher symmetries, their equivalent response is low dispersive [10].

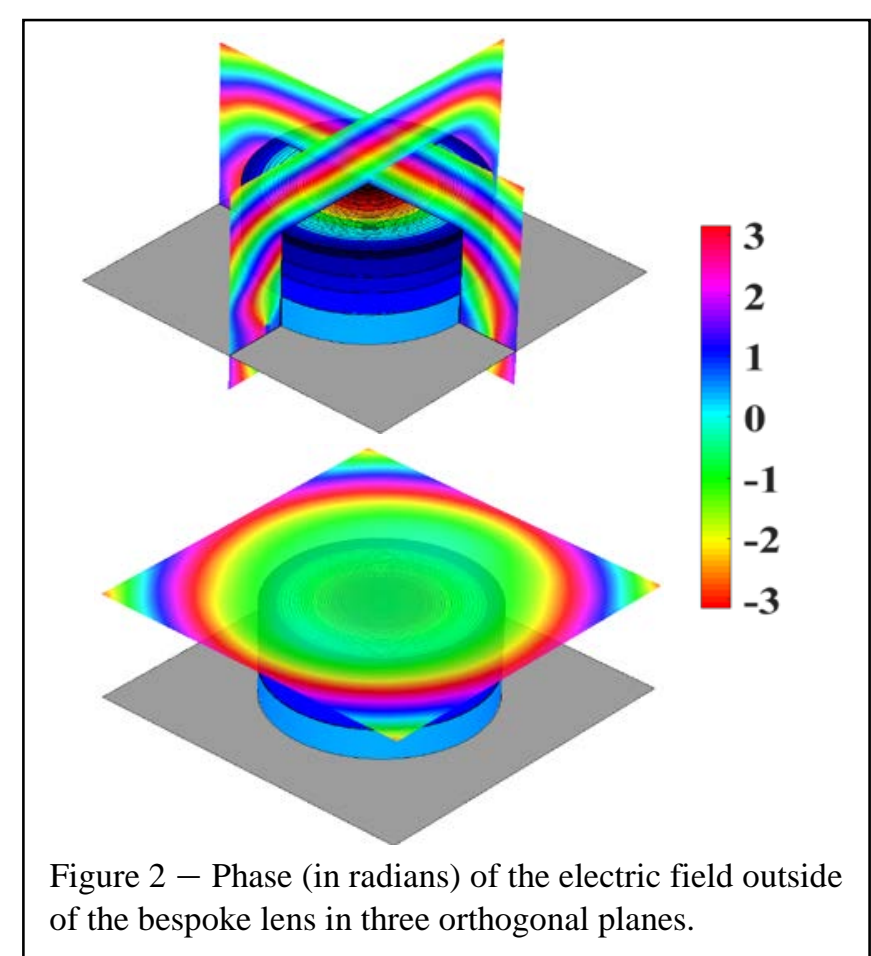

Therefore, these metasurfaces could be employed to produce ultra wide band lenses.

The second technological advance is the emergent expansion of 3D printers. In their first implementations, 3D printers were conceived to build mechanical objects. However, nowadays it is already possible to acquire 3D printers that make use of low loss dielectric materials. With the fast progress of 3D printers, the practical implementation of low cost and low loss bespoke lenses is becoming a reality. However, producing high resolution and smooth shapes is still a challenge. If the contours of the materials have roughness due to the low resolution of the printer, the quality of the overall antenna will be drastically affected at high frequencies. To overcome this problem, and to reduce the time of manufacturing that increases drastically the cost, in the future, it will be necessary to employ at least two resolutions to construct the diverse parts of the lens.

\section{Concluding Remarks}

Here, we have explained the concept of bespoke lens antennas. The 'bespoke' concept is a promising technique that can be employed to produce lenses adhoc for a given feeding antenna. Therefore, the overall antenna system is more directive than the original radiator. Furthermore, this technique can also be employed to tailor a radiation pattern. Practical demonstrators have not yet been built, but with the development of new 3D printers, which make use of microwave dielectric materials and higher symmetry metasurfaces, bespoke lenses have a propitious future. 


\section{References}

[1] U. Leonhardt, "Optical conformal mapping," Science, vol.312, no.5781, pp.1777-1780, 2006.

[2] D. Kwon and D. H. Werner, "Transformation Electromagnetics: An Overview of the Theory and Applications," IEEE Antennas Propagation Magazine, vol. 52, pp.24, 2010.

[3] D. A. Roberts, N. Kundtz, and D. R. Smith, "Optical lens compression via transformation optics", Optics Express, vol.17, no.19, pp.16535, 2009.

[4] R. C. Mitchell-Thomas, O. Quevedo-Teruel, T. M. McManus, S. A. R. Horsley, and Y. Hao, "Lenses on Curved Surfaces", Optics Letters, vol.39, pp.3551-3554, 2014.

[5] S. A. R Horsley, I. R. Hooper, R. C. MitchellThomas, and O. Quevedo-Teruel, "Removing Singular Refractive Indices with Sculpted Surfaces", Scientific Reports, vol.4, pp.4876, 2014.

[6] N. Kundtz and D. R. Smith, "Extreme-angle broadband metamaterial lens”, Nature Materials, vol.9, no.2, pp.129, 2010.

[7] O. Quevedo-Teruel, W. Tang, R. C. MitchellThomas, A. Dyke, H. Dyke, L. Zhang, S. Haq, and Y. Hao, "Transformation Optics for Antennas: Why limit the bandwidth with Metamaterials?" Scientific Reports, vol.3, pp.1903, 2013.

[8] Q. Wu, Z. H. Jiang, O. Quevedo-Teruel, J. P. Turpin, W. Tang, Y. Hao, and D. H. Werner, "Transformation Optics Inspired Multibeam Lens Antennas for Broadband Directive Radiation”, IEEE Transactions on Antennas and Propagation, vol.61, no.12, pp.5910-5922, 2013.

[9] M. Ebrahimpouri and O. Quevedo-Teruel "Bespoke Lenses Based on Quasi Conformal Transformation Optics Technique", IEEE Transactions on Antennas and Propagation, vol. 65, no. 5, pp. 2256-2264, May 2017.

[10] O. Quevedo-Teruel , M. Ebrahimpouri, M. Ng Mou Kehn, "Ultra Wide Band Metasurface Lenses Based on Off-Shifted Opposite Layers", IEEE Antennas and Wireless Propagation Letters, vol. 15, pp. 484487, 2016. 


\subsection{Transformation Optics for Antenna}

\section{Engineering - Yang Hao}

Queen Mary University of London

\section{Status}

Antennas have played an important role in many modern technological innovations ranging from Marconi's first transatlantic wireless transmission through Sir Henry Tizard's radar to modern cellular communications. Now enabled with two recent developments - transformations optics and the design and fabrication of novel electromagnetic materials, antenna engineers have been equipped with new design tools which provide entirely fresh solutions to classical problems restricted by fundamental physics such as the Chu-Harrington Limit in electrically small antennas, and enable new ways to manipulate the emission, propagation and absorption of EM radiation. This goes far beyond what can be accomplished with traditional materials in the form of lenses and mirrors, requiring both nano-composites and also those with properties that do not exist in nature (i.e., metamaterials [1]). TO has emerged as a new paradigm for EM design, providing equivalent material properties through a well-chosen change of coordinates, in order to achieve unprecedented wave manipulation [2, 3]. This is essential for the development of conformal antennas or flat panel antennas for both SATCOM and aerospace applications. The required material properties are complex (both permittivity and permeability are generally anisotropic and spatially varying). TO is at the heart of exciting ideas such as shaped reflectors and lens [4] with beam scanning and collimation capabilities while keeping low profiles and small RCS. Traditional phased arrays have limitations in wideangle beam-steering while TO based radome designs have opened up new possibilities to quest for low-cost and compact phased arrays. Earlier work of TO based antennas utilized fully benefits of metamaterials, which contain both electric and magnetic material properties being anisotropic and frequency-dispersive. Peculiar radiation performances can be achieved with the sacrifices in antenna gain as well as the bandwidth. Approximations can be made in several engineering oriented designs by restricting the use of no-resonating and magnetic metamaterials. The approach has led to the emergence of several novel lens antenna designs, notably a flat Luneburg lens [4]. Flat Luneburg lens antennas arise from industrial challenges on highly conformal and directive antennas, which are broadband, beam-steerable and possess low sidelobes under high power operations. The design methodology has consequently been applied to demonstrate antennas at high frequencies ranging from millimeter wave to optics. Quasi-conformal transformation optics, the idea

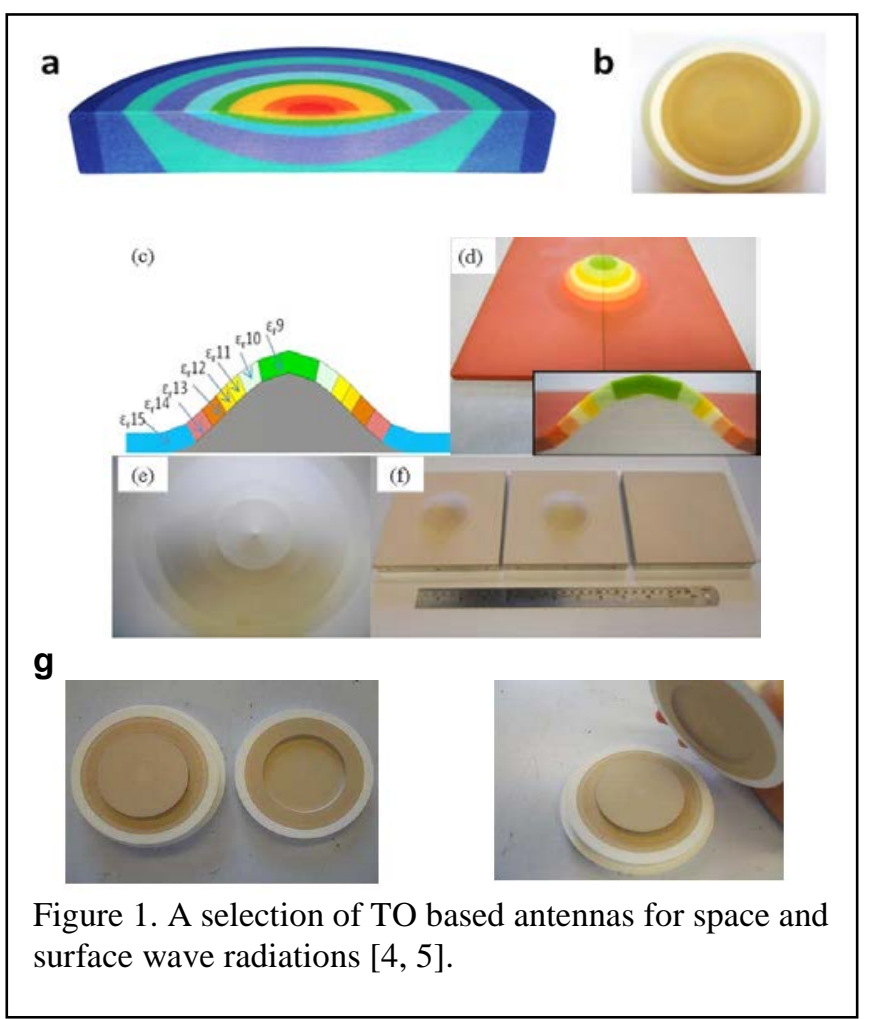

behind the design of carpet cloaks, has offered an alternative to TO antenna designs using all dielectric materials [5]. The paper published in IEEE Transactions on Antennas and Propagation introduces a robust engineering design approach supported with design examples of flat reflector and lens antennas [3]. In this paper, benefits of applying all dielectrics in antenna implementations have been fully quantified in order to offer broad operational bandwidths and such ideas have been further developed into the design of novel reflect-arrays. Further work has shown that future antennas may be made from structures similar to "LEGO" brick boxes and arbitrary radiation beams can be synthesized by dynamic changes of material properties of each building block. This concept complements so-called "coding metamaterials", "digital metamaterials" and "programmable metamaterials", which find numerous applications in radar, imaging and communications with the aid of advanced signal processing techniques such as "compressive sensing”.

Planar antennas remain to be a robust solution to many applications, including airborne radars not only for the design of phased arrays but also as surface wave radiators. To this end, TO provides a general method to achieve antennas and any other EM devices for surface waves, with the aim of linking the geometry and material coatings either isotropic or anisotropic over an arbitrarily, curved surface for all angles of incidence. The theory of TO for surfacewaves was presented in the design of cloak [2] and later experimentally demonstrated. This is a general theory that can be utilized for the design of conformal surface wave- 
based antenna devices, which are retrofitted to vehicles or airborne platforms, where perturbations of the surface are necessary for structural or aerodynamic reasons, but create scattering of the surface waves, which is detrimental to their performance. The concept has been consequently extended to the design of plasmonic metasurfaces and 2D materials for optical applications.

To make TO antennas with required exotic materials in large quantities, modern fabrication techniques will be needed, including the use of nano-composites and graded-index coatings [3, 8-10]. The permittivity values have been achieved using alternative dielectric mixtures with differing volume fractions and particle sizes ranging from nanometer to micron ranges. Proprietary titanate based ceramic materials were used and the device was fabricated using a series of novel techniques, which can be broken down into three distinct stages: particulate filler preparation, composite production and a multi-cast, sequential layer fabrication [3].

\section{Current and Future Challenges}

Recent UK-led breakthroughs in the theory of TO, such as the possibilities concerning cloaking and invisibility, have caught both the scientific and popular imagination, and have stimulated a huge growth in related research around the world. The potential of the underlying TO approaches however have much wider applicability than cloaking alone, in arguably more important applications that span communications, energy transfer, sensors and security. Under an EPSRC funded QUEST Programme Grant, a cross-disciplinary team was set up to include theorists, modellers, manufacturers and engineers who have been able to work together and bridge theory to manufacture and testing, with a clear focus on the reduction to practice and demonstration of potentially radical new concepts devices including antennas.

There is an industry-wide expectation that in order to meet the challenges of future aircraft communications systems radically novel design approaches are needed. Current designs of communication system are based on non-conformal solutions and/or mechanically steerable antenna systems using mechanically steerable parabolic dishes (bulky) or phased arrays (expensive), both covered with excessive radomes. These solutions are protuberant, increasing aerodynamic drag, fuel consumption, visibility, and degrading handling qualities.

To enable this step change, seamlessly embedded antennas are needed so that they become a part of the

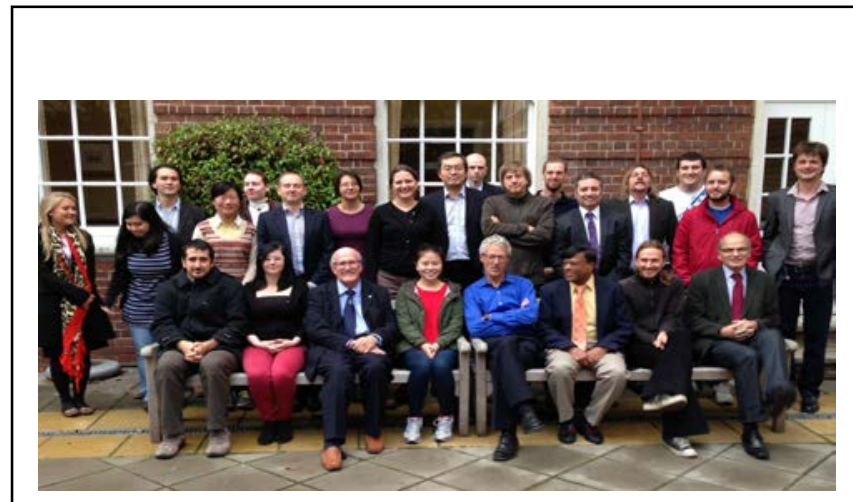

Figure 2 - A photo of EPSRC QUEST programme grant research team including some prominent members of scientific and industrial advisory team.

aircraft fuselage, which are constructed using advanced materials. This concept presents a highly innovative but challenging objective since solutions must also be manufacturable at reasonable cost while meeting structural and system functionalities. In addition, the effects of new functionalities on strict mechanical and safety performance must be considered, and MRObased repair and maintenance must remain possible. Engineering challenges relevant to the development of a product from its initial concepts to manufacturing will be addressed. This requires the translation of novel academic research into practical tools that can be easily adopted by industry.

\section{Advances in Science and Technology to Meet Challenges}

An adaptive approach to design is therefore required to optimize across (i) electromagnetic performance, (ii) aerodynamic performance based on realistic loads and non-linear vibrations and (iii) manufacturability, particularly drawing on latest 3D additive approaches to embedded functional materials. We aim to develop a novel computational tool-set that will be based on recent scientific advances in electromagnetics, atomistic-scale material and data-driven modeling both at the functional/structural dimensions and over the multi-scale geometric complexity. This deployment will provide robust design methodologies that can minimize the cost during the prototyping stage by providing results in a realistic time frame and will lead to optimal engineering designs in relation to aircraft that are ad hoc at best and heuristic at worst $[6,7]$.

Acknowledgments - The author would like to thank the Engineering and Physical Sciences Research Council (EPSRC), UK under a Programme Grant (EP/I034548/1) "The Quest for Ultimate Electromagnetics using Spatial Transformations (QUEST)'” for the funding, all researchers and 
industrial partners in the project team and all advisory board members for their contributions.

\section{References}

[1] Hao Y, Mittra R. FDTD modeling of metamaterials: Theory and applications. Artech house, 2008.

[2] RC Mitchell-Thomas, TM McManus, O QuevedoTeruel, SAR Horsley, Y Hao, "Perfect Surface Wave Cloaks”, Physical Review Letters 111 (21), 213901, 2013.

[3] Wenxuan Tang, Christos Argyropoulos, Efthymios Kallos, Wei Song, Yang Hao. "Discrete Coordinate Transformation for Designing All-Dielectric Flat Antennas.” IEEE Transactions on Antennas and Propagation vol. 58, (12) 3795-3804, 2010.

[4] Mateo-Segura C, Dyke A, Dyke H, Haq S, Hao Y. "Flat Luneburg Lens via Transformation Optics for Directive Antenna Applications”. IEEE Transactions on Antennas and Propagation, Volume:62, Issue: 4. 2014

[5] O. Quevedo-Teruel, W. Tang, R. C. MitchellThomas, A. Dyke, H. Dyke, L. Zhang, S. Haq, Y. Hao, Transformation Optics for Antennas: Why limit the bandwidth with Metamaterials?, Scientific Reports (Nature Publishing Group), vol. 3, pp. 1903, 2013.

[6] T. P. Runarsson and X. Yao, "Stochastic Ranking for Constrained Evolutionary Optimization,” IEEE Transactions on Evolutionary Computation, vol. 4, no.3, pp.284-294, 2000.

[7] B. Vial and Y. Hao, Topology optimized alldielectric cloak: design, performances and modal picture of the invisibility effect, Opt. Express, vol. 23, pp. 23551-23560, 2015.

[8] D.V. Isakov, , Q. Lei, F. Castles, C.J. Stevens, C.R.M. Grovenor, P.S. Grant, 3D printed anisotropic dielectric composite with meta-material features, Materials \& Design, vol. 93, pp. 423, 2016.

[9] F. Castles, D. Isakov, A. Lui, Q. Lei, C. E. J. Dancer, Y. Wang, J. M. Janurudin, S. C. Speller, C. R. M. Grovenor, P. S. Grant, Microwave dielectric characterisation of 3D-printed $\mathrm{BaTiO} 3 \mathrm{ABS}$ polymer composites, Scientific Reports 6, Article number: 22714 (2016).

[10] P. S. Grant, F. Castles, Q. Lei, Y. Wang, J. M. Janurudin, D. Isakov, S. Speller, C. Dancer, C. R. M. Grovenor, Manufacture of electrical and magnetic graded and anisotropic materials for novel manipulations of microwaves, Phil. Trans. R. Soc. A, vol. 373, pp 20140353, 2015. 


\section{Spacetime Cloaking}

\subsection{Spacetime Transformation Optics -} Paul Kinsler

Lancaster University

\section{Status}

Space-Time Transformation Optics (STTO) is a relatively new field of opportunity for electromagnetics researchers. It was initiated in 2011 with the first proposal for a STTO cloak, also described as an "event cloak" or "history editor" [1]. It was followed remarkably quickly by experimental demonstration [2] using the technologies of nonlinear optics and photonics. An STTO device is necessarily dynamic, but the modulated material properties were implemented not using exotic time-addressable metamaterial structures, but instead with so-called "time lenses" made from dispersion controlled optical fibres.

The easiest design principle for event cloaks, or indeed any STTO device, is that of speed control of the illumination. By ensuring none of this background light illuminates the selected event, but guaranteeing that it nevertheless departs the device it as if it would have, the space-time cloak carries out its history editing trick. Most simply, in the 1+1D example shown in figure 1, if earlier (later) parts of the illuminating wave travel faster (slower) than normal, a dark region of "shadow" opens up, in which un-illuminated - and therefore unseen - events can take place. Then, with the speed modulation reversed, the gap can be closed.

Experimental schemes for space-time cloaking distinct from the first example also exist: the most applications-oriented being based on either the timedomain Talbot effect [3], or the recent application using temporal Fraunhofer diffraction [4]. Competing approaches have involved accelerating wave packets, Fourier analysis, or polarization bypass, and indeed other schemes, although in some cases the definition of what space-time cloaking means has perhaps been over-stretched.

Beyond the idea of cloaking as an end in itself, in ordinary spatial transformation optics we also have carpet cloaking and exterior cloaking [5]. Work on space-time carpet cloaking already exists [6], but it demands independent bi-directional material properties, which are hard to implement. Relativistic movement of cloaks, movement being the most straightforward of space-time transformations, has also been addressed [7]. But it remains an interesting question as to whether a space-time exterior cloak can even make sense as a concept.

We can therefore see that the current state of play has the theory of STTO being well understood, and is easily advanced enough to get things done, as demonstrated by experiments that produce either individual or (more usefully) periodic streams of cloaks, and that even work at telecommunications data rates. Further, some quite general explorations of future possibilities for STTO have been written $[8,9]$. But what next?

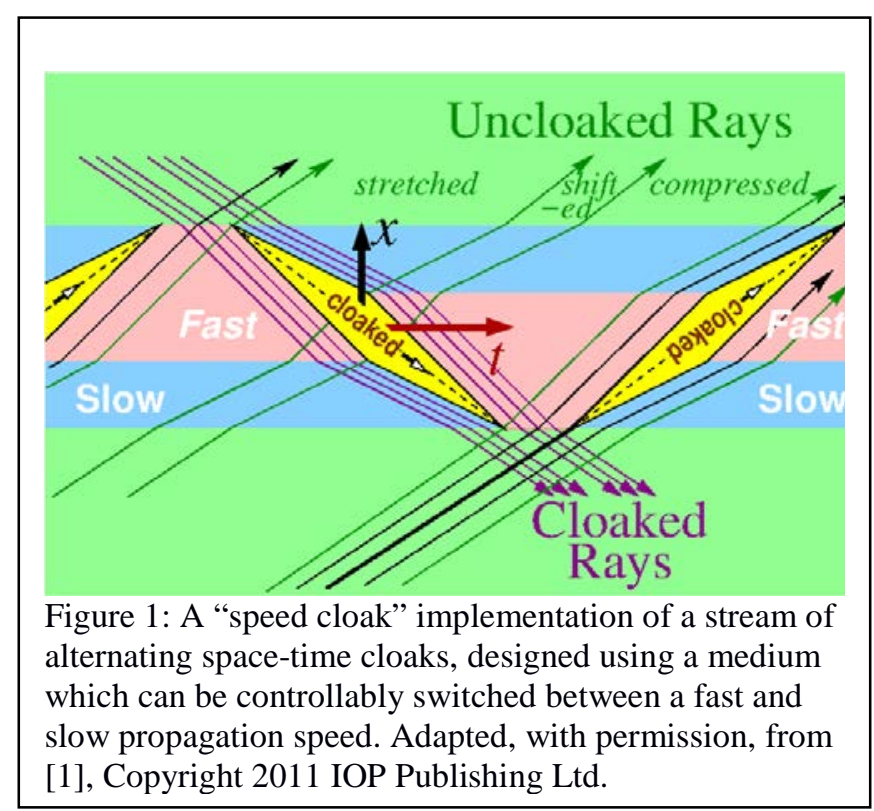

\section{Current and Future Challenges}

Given the current existence of space-time cloaking apparatus operating at telecommunications data rates [3], one might wonder whether that, from the point of a physicist, the challenges to the field are now firmly within the scope of engineering. However, despite what engineers might have planned, this would be an over-optimistic assessment. From the point of view of physics, there remain two significant challenges to the field. As it stands, current implementations of event cloaks are basically "speed cloaks" [8], which, whilst working perfectly well, implement an approximate form of the cloaking transformation.

A more correct treatment of STTO [10] that allows for the effect of dispersion strongly pushes us towards the use quite sophisticated mathematics; i.e. the language of differential forms. This is something of a barrier to wider investigation of STTO, since these mathematical skills are less prevalent in the transformation optics community, which more usually relies on vector or tensor-based formalisms. In such standard approaches, particularly the vectorial one, it can be difficult to avoid implicit or hidden assumptions. Nevertheless, there is still significant scope for STTO design based on the simple speed-modulation analogies, it is worth noting that space-time transformations require more 
thought than simply building a device with a dynamic and controllable response.

Beyond the mathematical demands of understanding STTO, there are the technological necessities of building devices. For the implementation of a metamaterial-based space-time cloak we need metamaterial unit cells that can not only have their response modified dynamically [11], but which are easily addressable and can be controlled within precise timing requirements. Given that the unit cell responses have to be modulated along with the illuminating light as it propagates, this is particularly challenging for electromagnetic signals.

Another puzzle is what STTO will actually be useful for - especially when we decide to think beyond the scope of straightforward research demonstrators and towards engineering applications. Perhaps some already exist - the time lenses used in the STTO experiment could simply be rebadged as STTO elements, if the time lens operation was just recast as being designed from an appropriate transformation. However, we will only advance our understanding and technology by adding something new and concrete, rather than by claiming progress based on a mere renaming process, however aptly chosen it might seem.

\section{Meeting the Challenges}

The first ST cloak paper [1] proposed an optical fibre implementation, suggesting that a dynamically modulated refractive index profile controlled by nonlinear refractive index manipulation. Of course, this was a theorists' suggestion, so it should be no surprise that when the actual experiments appeared, they did things more elegantly, with dispersion engineered time-lenses [2]. In terms of nonlinear optics implementations, it seems that to a large extent the technology is more than up to current challenges.

However, traditional TO implementations typically use metamaterials, engineered unit cells assembled in arrays to set up the necessary spatial properties, and sometimes modulation properties. Is there scope for this kind of approach to building space-time cloaks? The timing challenges alone would be tremendous, although would be easier if built in a high index background context. Given those difficulties, an acoustic ST cloak, with its lower frequencies and slower wave speeds may be the correct angle to take, since EM/electronic signaling speeds far outstrip the speed of sound; or maybe there is scope for working at longer wavelengths, in the microwave regime, in order to simplify fabrication.

A recent review reports [11] electrically tunable metamaterials from the radio frequency into the infrared, at modulations up to $\mathrm{THz}$ frequencies; and also success with mechanically, thermally, and optically tunable metamaterials cells. However, it is not clear how these might be integrated into something with the precise timing requirements of an event cloak.

Generally, STTO implementations are of the approx "speed cloak" sort, and ignore the complications of the transform's effects on temporal and spatial dispersion. Estimates [10] show that in the optical range, these effects are likely to be very small, since bandwidths tend to be narrow and the speed of light is very fast. Here acoustic implementations may again be useful, by enabling greater scope for experimental probing of these subtleties.

There remain other suggestions from the original paper that as yet lack experimental implementation [1]: perhaps most notably the teleport illusion, and the signal processing 'interrupt-without-interrupt"' idea. Both do not obviously require a great improvement in experimental technology, but do need to replace a series of one-off (disconnected) events with an ordered stream of related events to manipulate. With such a stream of events, it would even be possible to try to build the causality editor [8] extension of the history editor concept [1].

The mathematics required to treat approximate models of STTO devices is neither too abstract or too difficult in comparison to typical theoretical approaches, although particular calculations might contain many terms, or features difficult to simplify. However, a rigorous treatment is far more demanding, as demonstrated recently by Gratus et al [10]. Further, although adding the consideration of static curvature into such calculations is not a great complication, if one were to attempt a STTO design in a full dynamical spacetime - i.e. a general relativistic formulation then. One item that needs to be addressed is therefore how to make the advanced mathematics either more palatable or more accessible to the wider research community.

\section{Concluding Remarks}

In this section I have reflected on some of the recent developments in space-time transformation optics. The promising start, where the second experimental paper noted operation of devices with "telecoms data rates" looks set to continue. However, the arrivals of interesting new implementations have yet to become a flood, so it seems clear at the moment that there remains a lot of scope for progress in research, as well as development of real-world applications.

Acknowledgments - I would like to acknowledge discussions with MW and JG; along with funding from STFC (the Cockcroft Institute ST/G008248/1) and 
EPSRC (the Alpha-X project EP/J018171/1 and EP/N028694/1.)

\section{References}

[1] McCall M W, Favaro A, Kinsler P, Boardman A D, 2011, A spacetime cloak, or a history editor J. Opt. 13024003

[2] Fridman M, Farsi A, Okawachi Y and Gaeta A L, 2012, Demonstration of temporal cloaking Nature $\mathbf{4 8 1}$ 62-65

[3] Lukens J M, Leaird D E, Weiner A M, 2013, A temporal cloak at telecommunication data rate Nature 498 205-208

[4] Zhou F, Dong J, Yan S, Yang T, 2017, Temporal cloak with large fractional hiding window at telecommunication data rate, Opt. Comm. $\mathbf{3 8 8} 7783$

[5] Lai Y, Chen H, Zhang Z-Q, Chan C T, 2009, Complementary media invisibility cloak that cloaks objects at a distance outside the cloaking shell, Phys. Rev. Lett. 102093901

[6] Kinsler P, McCall M W, 2014, Transformation devices: carpets in space and time, Phys. Rev. A 89063818

[7] Halimeh JC, Thompson RT, Wegener M Invisibility cloaks in relativistic motion, Phys. Rev. A 93013850

[8] Kinsler P, McCall M W, 2014, Cloaks, editors, and bubbles: applications of spacetime transformation theory, Ann. Phys. (Berlin) 526 51-62

[9] Kinsler P, McCall M W, 2015, The futures of transformations and metamaterials, Photon. Nanostruct. Fundam. Appl. 15 10-23

[10] Gratus J, Kinsler $P$, McCall $M \mathrm{~W}$, Thompson R T, 2016, On spacetime transformation optics: temporal and spatial dispersion New J Phys 18123010

[11] Fan K, Padilla W J, 2015, Dynamic electromagnetic metamaterials Materials Today 18 39-50 


\subsection{Transformation optics with spatial dispersion -} Jonathan Gratus, Lancaster University and Cockcroft Institute.

\section{Status}

Combining spatial dispersion (also known as nonlocal effects) with transformation optics is relatively new area of research and there is not much literature to date. In $[1,2]$ the authors consider plasmons on touching and nearly touching nano wires and use conformal transformation optics. They need to consider spatial dispersion, since it limits the maximum fields one may see in such a scenario. In [3] the authors consider the fields due to a nano sphere in contact with a surface in which surface plasmons are transmitted. In all these cases spatial dispersion arises naturally due to the limited comprehensibility of the electrons. In [4] the authors consider creating a spatially dispersive media in order to create nonreciprocal media, leading to ultratransparent material. In [5,6] the authors consider homogeneous media and use spatial dispersion in order to manipulate the dispersion relations. In [7] the authors consider the transformation optics of a spacetime cloak and spatial dispersion arises naturally as the result of the transformation on a temporally dispersive pulse.

Spatial dispersion is usually referred to when the constitutive relations (permittivity, permeability and magnetoelectric effects) depend on the wavevector $\boldsymbol{k}$ in addition to the frequency $\boldsymbol{\omega}$. It occurs when the polarization $\boldsymbol{P}$ at one point $\boldsymbol{x}$ depends not only on the electric field at $\boldsymbol{x}$ but also on the electric field in a neighbourhood of $\boldsymbol{x}$. Some authors also talk about weak spatial dispersion, which is the result of reformulating a non spatially dispersive magnetoelectric medium. Here we are concerned with strong spatial dispersion and consider magnetoelectric effects separately.

In metamaterials spatial dispersion is often considered a nuisance and occurs when the size $\boldsymbol{L}$ of the microcells starts becoming comparable with the wavelength $\lambda$. As a rule of thumb, one may say that the constitutive relations of metamaterial are temporally dispersive when $\boldsymbol{L} \lesssim \lambda / \mathbf{1 0}$, and is both spatially and temporally dispersive when $\boldsymbol{\lambda} / \mathbf{1 0} \lesssim \boldsymbol{L} \lesssim \boldsymbol{\lambda} / \mathbf{2}$. For $\boldsymbol{L}>$ $\lambda$ the use of effective media approximations is no longer valid. Note that for reasons of causality, spatially dispersive implies temporally dispersive. Therefore saying a medium is spatially dispersive means both. The simplest constitutive relation, which incorporates spatial dispersion is the hydrodynamic Lorentz model which has $\boldsymbol{\mu}=\boldsymbol{\mu}_{\mathbf{0}}$ and

$$
\varepsilon(\omega, k)=\varepsilon_{0}+\frac{\omega_{P}^{2}}{-\omega^{2}+i \gamma \omega+\omega_{0}^{2}+\beta_{x}^{2} k_{x}^{2}}
$$

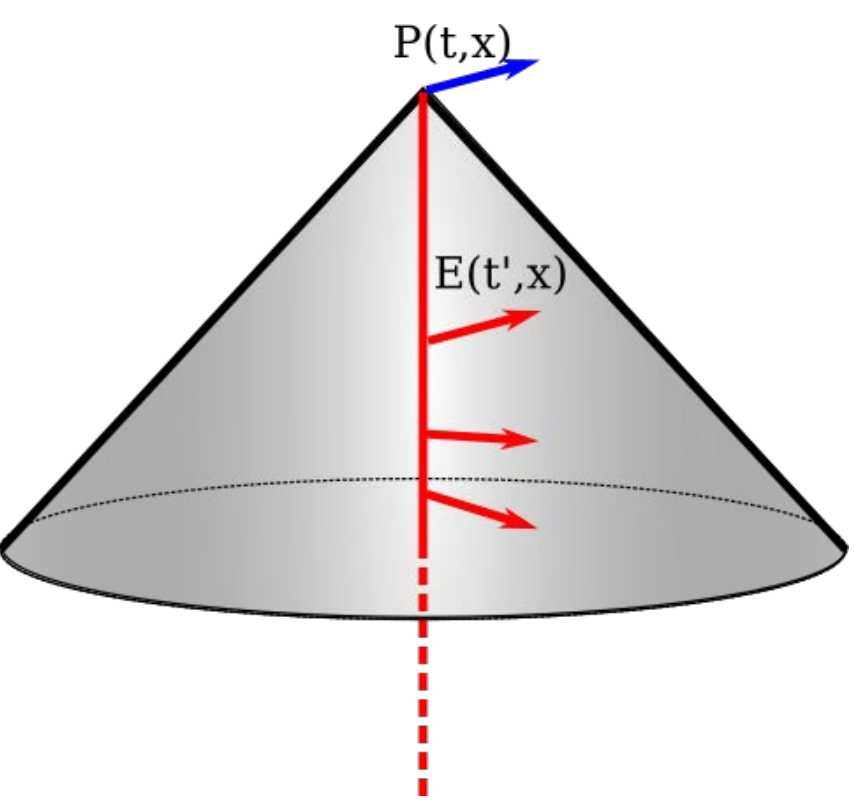

Figure 1: The support (red) of the integral kernel associated with a temporally dispersive medium, inside the backward lightcone (grey). The Polarisation

$\boldsymbol{P}(t, \boldsymbol{x})$ depends on the electric field $\boldsymbol{E}\left(t^{\prime}, \boldsymbol{x}\right)$ in the its past, at the point $\boldsymbol{x}$ onlv.

By its very nature transformation optics leads to media with constitutive relations which depend on position. Together with spatial dispersion, this requires prescribing a constitutive relation which depends on both $\boldsymbol{x}$ and $\boldsymbol{k}$. Since these are Fourier conjugate variables, it appears to be inconsistent to depend on both. There are a number of ways handling this: In most work [1-3] various scale-dependent approximations are used to decouple $\boldsymbol{k}$ and $\boldsymbol{x}$, so that it is reasonable for the constitutive relation to depend on both. For example we might assume that $\boldsymbol{k} \cdot(\boldsymbol{\delta} \boldsymbol{x}) \ll \mathbf{1}$ where $\boldsymbol{\delta} \boldsymbol{x}$ is the size over which significant changes in the constitutive relations occur. By contrast, others [46] consider constitutive relations which depend only on $(\boldsymbol{\omega}, \boldsymbol{k})$. Thus they consider homogeneous regions of space. In general the solutions in each region will require additional boundary conditions to match [8].

A more accurate method is to write the constitutive relations either as an integral kernel $[9,10]$ or as a differential equation [7]. Example kernels associated with local and nonlocal constitutive relations are given in figures 1 and 2 . The hydrodynamic model becomes a differential equation for the polarization $\boldsymbol{P}(\boldsymbol{t}, \boldsymbol{x})$

$$
\frac{\partial^{2} P}{\partial t^{2}}+\gamma \frac{\partial P}{\partial t}+\omega_{0}^{2} P-\beta_{x}^{2} \frac{\partial^{2} P}{\partial x^{2}}=\omega_{P}^{2} E(t, x)
$$

In both of cases the constitutive relations can be generalised, so that they can be interpreted as depending both on $\boldsymbol{k}$ and $\boldsymbol{x}$. Thus in the differential 
The use of general relativity, inherent in transformation optics, implies that the tools can be easily extended to spacetime transformations, as required for spacetime cloaking. These leads to constitutive relations which depend on which event in spacetime is chosen. In this case the constitutive relations need to depend of $(\boldsymbol{t}, \boldsymbol{x})$ and their Fourier conjugates $(\boldsymbol{\omega}, \boldsymbol{k})$. Further, when generalising the PDE above, one has to decide whether to use Lie derivatives or covariant derivatives. In [7] the authors chose to use the Lie derivative. This should be considered as part of the constitutive relations, and would have to be determined experimentally. This will require a moving medium or a strong gravitational field, since both types of derivatives reduce to the same partial derivatives in the static case.

In [7] the authors derive the full constitutive relations in terms of differential equations, and then make the "slowly varying envelope approximation" and the "gradual transformation approximation" in order to find a similar expression. By contrast others [1-3] use an approximation scheme from the outset. A theoretical challenge therefore would be to show that such an ab initio approximation scheme is valid for all systems by deriving it from the full system.

\section{Current and Future Challenges}

Up to now spatial dispersion has usually been considered an annoyance. Researches have tried to manufacture materials which minimise its effects. The next major step will require the manufacturing of materials in which the desired spatial dispersion is designed into the material.

Devices that implement the approximation where $\boldsymbol{k}$. $(\boldsymbol{\delta} \boldsymbol{x}) \ll \mathbf{1}$ are foreseeable. One challenge therefore is to design microcells which result in a prescribed spatial dispersion, for example by including a wire medium. It is not unreasonable to consider these structures to be dynamic, enabling the creation of spacetime cloaks which can hide dispersive pulses.

A longer term challenge will be to create materials which implement the approximated constitutive relations. This is likely to be an order of magnitude harder.

In terms of numerical simulations there is a major challenge in calculating effective spatially dispersive constitutive relations for a given unit cell. Most existing software can use a frequency domain solver for finding the dispersion relation and a time domain solver for finding the transmission and reflection coefficients. If a medium is not spatially dispersive (and not magnetoelectric) there is a one to one

relationship between the dispersion relation and the refractive index. The transmission and reflection

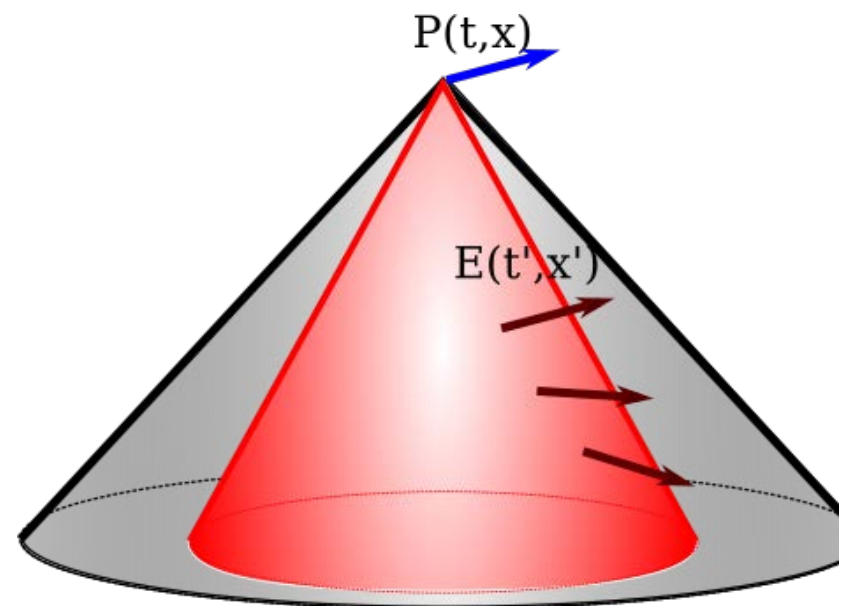

Figure 2: The support (red) of the intergral kernel associated with a temporally and spatially dispersive medium, inside the backward lightcone (grey). The Polarisation $P(t, \boldsymbol{x})$ depends on the electric field $E\left(t^{\prime}, \boldsymbol{x}^{\prime}\right)$ in the its past lightcone.

coefficients can then be used to calculate the impedance, so together one can uniquely determine the permittivity and permeability. By contrast, if there is spatial dispersion no such calculation can be made. Consequently, the dispersion relation can give definitive proof that spatial dispersion is present. For example $\mathrm{A}$ : if the equi-frequency plots are not symmetric, or B: if there are multiple wavevectors $\boldsymbol{k}$ which solve the dispersion relation, which have the same frequency, direction and polarization. However the dispersion relations cannot tell us what the constitutive relations are. For example, for a linearly polarised wave travelling in a homogeneous isotropic spatial dispersive medium $\boldsymbol{\varepsilon}(\boldsymbol{\omega}, \boldsymbol{k})$ and $\boldsymbol{\mu}=\boldsymbol{\mu}_{\mathbf{0}}$ and no magnetoelectric effect, then Maxwell's equations give the dispersion relation $\boldsymbol{\omega}=\boldsymbol{\Omega}(\boldsymbol{k})$ where $\boldsymbol{\Omega}(\boldsymbol{k})^{2} \mu_{\mathbf{0}} \boldsymbol{\varepsilon}(\boldsymbol{\Omega}(\boldsymbol{k}), \boldsymbol{k})=\boldsymbol{k}^{2}$. It is easy to see that one can replace $\varepsilon(\boldsymbol{\omega}, \boldsymbol{k})$ with $\varepsilon(\boldsymbol{\omega}, \boldsymbol{k})+\boldsymbol{a}(\boldsymbol{\omega}, \boldsymbol{k})\left(\boldsymbol{k}^{2}-\right.$ $\left.\boldsymbol{\omega}^{2} \mu_{0} \varepsilon(\boldsymbol{\omega}, \boldsymbol{k})\right)$ without changing the dispersion relation. Thus a major advance would be to develop a piece of software, in which one inputs a unit cell, and it outputs the effective constitutive relations, including any magnetoelectric effects.

A further challenge is to see if one can find the additional boundary conditions needed to implement an edge to the medium. This will probably involve the combination of a homogeneous metamaterial with a metasurface, representing the interface, both of which may be spatially dispersive.

\section{Concluding Remarks}

Using spatial dispersion in transformation optics gives many exciting opportunities, both to understand better 
scenarios where nonlocal effects occur naturally and also to introduce them to create new applications. There are significant theoretical, numerical and manufacturing challenges, some of which we have highlighted.

Acknowledgments - The author is grateful for the support provided by STFC (the Cockcroft Institute ST/G008248/1 and ST/P002056/1) and EPSRC (the Alpha-X project EP/J018171/1 and EP/N028694/1).

\section{References}

[1] Fernández-Domínguez AI, Zhang P, Luo Y, Maier SA, García-Vidal FJ and Pendry JB. Transformation-optics insight into nonlocal effects in separated nanowires. Physical Review B, 86(24):241110, 2012.

[2] Fernández-Domínguez AI, Wiener A, García-Vidal FJ, Maier SA and Pendry JB 2012 Transformationoptics description of nonlocal effects in plasmonic nanostructures Physical review letters 108(10):106802, 2012.

[3] Cirací C, Hill RT, Mock JJ, Urzhumov Y, Fernández-Domínguez AI, Maier SA, Pendry JB, Chilkoti A and Smith DR 2012 Science, 337(6098):1072--1074

[4] Luo J, Yang Y, Yao Z, Lu W, Hou B, Hang ZH, Chan CT and Lai Y Ultratransparent media and transformation optics with shifted spatial dispersions 2016 Physical review letters 117(22):223901

[5] Castaldi G, Galdi V, Alù A and Engheta N 2012 Nonlocal transformation optics Physical review letters 108(6):063902

[6] Moccia M, Castaldi G, Galdi V, Alù $A$ and Engheta N 2016 Dispersion engineering via nonlocal transformation optics Optica 3(2):179--188

[7] Gratus J, Kinsler P, McCall MW and Thompson RT 2016 On spacetime transformation optics: temporal and spatial dispersion New Journal of Physics 18(12): 123010

[8] Pekar SI 1958 The theory of electromagnetic waves in a crystal in which excitons are produced Sov. Phys. JETP 6(4):785

[9] Agranovich VM and Ginzburg V 2013 Crystal optics with spatial dispersion and excitons volume 42 Springer Science \& Business Media

[10] Gratus J and Tucker RW 2011 Covariant constitutive relations and relativistic inhomogeneous plasmas Journal of Mathematical Physics 52(4):042901 
7.3 Experimental progress in temporal cloaking Joseph M Lukens ${ }^{1}$ and Andrew M Weiner ${ }^{2}$

${ }^{1}$ Oak Ridge National Laboratory

${ }^{2}$ Purdue University

\section{Status}

It is certainly not surprising that experiment has trailed theory for the exotic spacetime cloaks introduced earlier in this roadmap. Nonetheless, experiments have advanced with remarkable speed, with several temporal cloaks already demonstrated and exciting possibilities ready to be explored. Though not essential to cloaking as a concept, spacetime duality [1] has proven an important catalyst in the rapid development of temporal cloaks. In contrast to the use of "spacetime" in "spacetime cloak" as an integration of spatiotemporal characteristics, spacetime duality distinguishes space and time in a useful way, by noting the formal correspondence between spatial Fourier optics and narrowband temporal dispersion. In the case of temporal cloaking in particular, such duality helps demystify the notion of hiding transient events by supplying more tangible spatial equivalents. Indeed, the first demonstrated temporal cloak can be represented by the temporal ray diagram in Fig. 1 [2]. A monochromatic probe field receives a sharp chirp discontinuity via nonlinear optical wave mixing with a tailored pump pulse; by propagating through a dispersive element, the frequencies separate and leave a time hole with zero intensity, wherein any event has no impact; then finally, moving through the matched system returns the probe to its initial undisturbed state, with no trace of the event. This seminal experiment succeeded in cloaking a four-wave mixing signal from the probe field and confirmed, within just one year from its initial proposal, that time cloaking is possible in a fiber-optic system.

Shortly thereafter emerged an alternative temporal cloaking scheme based on electro-optic modulation rather than nonlinear mixing [3]. Whereas both nonlinear optical mixers and electro-optic modulators implement the same physical operation-namely, temporal phase modulation - they do so in very distinct ways in terms of execution and performance. Accordingly, this telecom-compatible, electro-optic approach was able to boost the repetition rate by several orders of magnitude, into the gigahertz regime, and produced time gaps equal to roughly one halfperiod; together these enabled cloaking of high-speed optical data streams for the first time, extending time cloaking to the realm of telecommunications-a fitting application space for clandestine cloaks. Interestingly, this demonstration also enlisted spacetime duality via the temporal Talbot effect, using interference to produce temporal gaps at high speeds without any

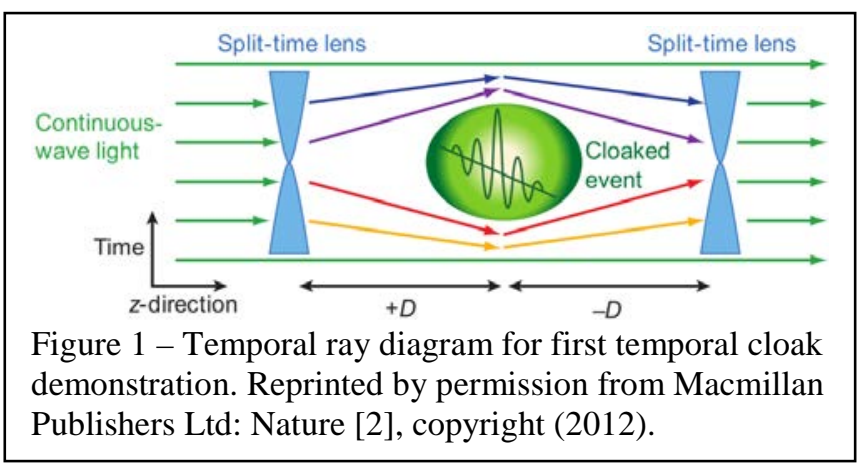

chirp discontinuities. Combined with the original time cloak [2], these two proof-of-principle experiments form the foundation on which subsequent temporal cloaks can build.

\section{Current and Future Challenges}

Despite the brisk pace of the foundational experiments, time cloaking remains far from ideal, particularly in its restriction of cloakable probe fields and events. For example, both initial cloaks [2,3] accept only a monochromatic probe which-while an excellent benchmark case, containing no initial time gaps-is certainly not arbitrary. Similarly, time gaps approaching the full modulation period are essential for expanding the range of events which can be cloaked. On a technical side, recent developments have brought improvements to the cloaking window question. In [4], a time gap approaching $90 \%$ was obtained using highly nonlinear fiber for spectral broadening. This approach represents an interesting hybrid of the first two temporal cloaks, combing the electro-optic modulation of [3] for high data rates with the optical nonlinearity of [2] for short-pulse compression. On another front, the cloaking window was increased not by pulse compression for a fixed period, but by repetition-rate reduction for fixed pulse width, using the extended Talbot effect-similar to pulse picking but with no intrinsic loss [5]. These experiments indicate promise in realizing fractional cloaking windows approaching $100 \%$, although we anticipate diminishing returns for each step toward this limit, with small improvements requiring significant increases in system complexity. Finding new technological approaches may be required to achieve such limits.

The second outstanding challenge relates to the arbitrariness of the probe fields which a temporal cloak can manipulate. Up to this point, the experimental focus has been on continuous-wave inputs at a given frequency; by spacetime duality, the spatial equivalent would be a cloak which hides an object from a plane wave at one angle of illumination only. Just as a unidirectional cloak is only a penultimate realization of spatial cloaking, temporal cloaks which can expand from single-frequency to more arbitrary inputs represent a crucial goal in the coming years. Closely 
related is an even more fundamental question: How can we make current temporal cloaks viable in tackling practical problems? For example, the standard paradigm described above does indeed enable secret disruption of communication, but offers no positive way to transmit the cloaked information. Nonetheless, important first steps addressing these broad challenges have been realized. Building on the same platform as [3], an improved setup exploited the spectrally periodic nature of the temporal Talbot effect to accept multiple probe wavelengths; as shown in Fig. 2, one channel can be cloaked, while the other faithfully conveys the data stream [6]. Moreover, this configuration succeeded in taking a modulated probe input and transmitting it through an event unscathed, demonstrating a novel form of tampering resistance. These experiments not only reveal cloaking effectiveness with polychromatic inputs, but they also provide concrete examples of time cloaks used to improve, rather than interrupt, communication. We also note an experiment employing polarization switching to hide or transmit high-speed data [7]. While not temporal cloaking in the true sense (opening gaps in time), this polarization switch suggests an important principle when designing time cloaks for applications: the experimental means is secondary to the application - that is, one should focus on achieving a given result, rather than adhering to a particular theoretical pattern. In light of this second wave of experiments, for the near term we see temporal cloaking best suited to specific optical communication tasks, on an application-by-application basis. The more challenging, long-range trajectory toward (semi-) universal communication cloaks then can be pursued in parallel.

Finally, the greatest-and by far most uncertainchallenge for the time cloak experimentalist is uniting the aforementioned techniques with metamaterials, in order to realize a true spacetime cloak. All temporal cloaks thus far can be classified as spatially onedimensional, creating time gaps in a single spatial mode. And no explicit proposal for integrating spatial and temporal cloaks has been offered, at least in the form of a prescription fulfillable with current technology. This is not horribly surprising, given the technological dissimilarity between metamaterial approaches (for spatial cloaks) and high-speed fiber optics (for temporal cloaks). Yet if the history of transformation optics has taught us anything, it is never to eliminate fantastic possibilities, for one never knows when an unexpected breakthrough might turn science fiction into reality.

\section{Advances in Science and Technology to Meet Challenges}
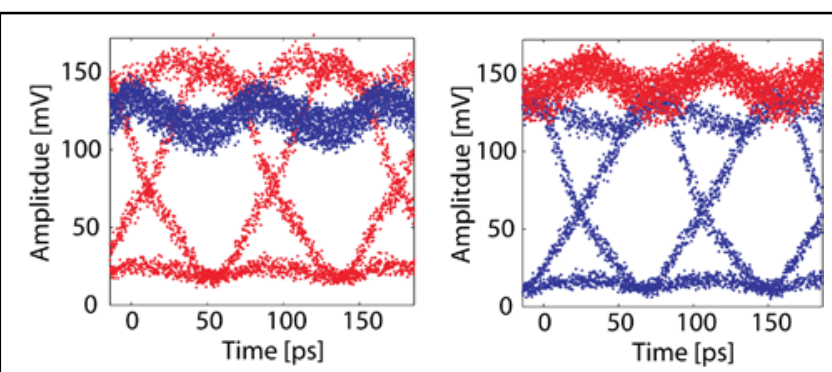

Figure 2 - Simultaneous cloaking and data transmission for wavelength-multiplexed channels. Cloaking along the short-wavelength (blue) channel (left) or along the long-wavelength (red) channel (right). Reproduced with permission of OSA from [6].

We note one intriguing connection for improving time cloaking, deriving from a somewhat unlikely source: quantum information processing (QIP). QIP utilizes quantum states, coherent manipulations, and entanglement in order to realize certain informational tasks more effectively than possible in classical systems. Though typically not associated with cloaks designed for classical electrodynamics, photonic QIP requirements share much in common. For example, a successful QIP system necessitates arbitrary and unitary optical mode transformations; likewise, the transformation for an ideal cloak directs arbitrary inputs away from a particular cloaked region, and then transforms back to the input, all unitarily (i.e., without loss). In time-frequency space, the degrees of freedom relevant to temporal cloaking, recent research has theoretically developed universal quantum computing based on frequency-encoded photons, pulse shapers, and electro-optic phase modulators [8] - the very spectro-temporal phase operations on which previous linear temporal cloaks have relied. The same operations form the basis for new entanglement tests for frequency-entangled photons [9]. And since universal unitary operations encompass those needed for time cloaking, this advance in photonic QIP presents a concrete tool for cloak design. While these QIP results apply specifically to discrete optical mode spaces (like wavelength-multiplexed networks), they should generalize to continuous spaces as well; developing such a paradigm in more detail is an important area for future work.

\section{Concluding Remarks}

Temporal cloaking experiments have progressed steadily following their initial proposal, with fiberoptic instantiations attaining high-speed data cloaking, and important progress in communication-enabling as well as communication-thwarting systems. Technical improvements to current cloak designs are ongoing; expanding time cloaks to more general probe waveforms is promising; and experimental realization of a full spacetime cloak represents an exciting though 
still largely indefinite prospect. Yet regardless of how the field progresses in the coming years, time cloak experiments have already revealed valuable connections between transformation optics, optical communications, and even quantum informationlinks which bring new insights into all fields involved and suggest unique opportunities for improving realworld applications.

Acknowledgments - This work was performed in part at Oak Ridge National Laboratory (ORNL), operated by UT-Battelle for the U.S. Department of Energy under contract no. DE-AC05-00OR22725. JML is funded by a Wigner Fellowship at ORNL. AMW recognizes support from the National Science Foundation under grants ECCS-1407620 and ECCS1509578.

\section{References}

[1] Kolner B H 1994 Space-time duality and the theory of temporal imaging IEEE J. Quantum Electron. 30 1951-63

[2] Fridman M, Farsi A, Okawachi Y, and Gaeta A L 2012 Demonstration of temporal cloaking Nature $48162-5$

[3] Lukens J M, Leaird D E, and Weiner A M 2013 A temporal cloak at telecommunication data rate Nature 498 205-8

[4] Zhou F, Dong J, Yan S, and Yang T 2017 Temporal cloak with large fractional hiding window at telecommunication data rate Opt. Commun. 388 77-83

[5] Li B, Wang X, Kang J, Wei Y, Yung T, and Wong K K Y 2017 Extended temporal cloak based on the inverse temporal Talbot effect Opt. Lett. 42 767-70

[6] Lukens J M, Metcalf A J, Leaird D E, and Weiner A M 2014 Temporal cloaking for data suppression and retrieval Optica $1372-5$

[7] Bony P-Y, Guasoni M, Morin P, Sugny D, Picozzi A, Jauslin H R, Pitois S, and Fatome J 2014 Temporal spying and concealing process in fibreoptic data transmission systems through polarization bypass Nature Commun. 54678

[8] Lukens J M and Lougovski P 2017 Frequencyencoded photonic qubits for scalable quantum information processing Optica 4 8-16

[9] Imany P, Jaramillo-Villegas J A, Odele O D, Han K, Qi M, Leaird D E, and Weiner A M 2017 Demonstration of frequency-bin entanglement in an integrated optical microresonator CLEO JTh5B.3 [postdeadline] 


\section{Transformation Optics for Analogue Cosmology}

\subsection{Cosmology in the laboratory: challenges at the} horizon - Ulf Leonhardt

Weizmann Institute of Science

\section{Status}

An important inspiration for transformation optics has been the connection between electromagnetism in media and general relativity [1]. This connection is based on the long-known mathematical fact [2] that Maxwell's equations in curved coordinates or curved space-time geometries (Fig. 1) are equivalent to Maxwell's equations in certain impedance-matched magneto-electric media. One can therefore make use of some of the concepts of general relativity in electromagnetism. In particular, the concept of coordinate-transformations and coordinate invariance has been rather fruitful in what became known as transformation optics. Invisibility cloaking by spatial transformations [1], for example, is simply a consequence of coordinate invariance: as Maxwell's equations are coordinate-invariant, the effect of a coordinate-transformation - implemented with an appropriately-designed medium - is invisible, provided the transformation does not affect the observer. The question is whether macroscopic electromagnetism can pay back some the debt it owes to general relativity. Thanks to the recent detection of gravitational waves [3] and to other advances in instrumentation and technique [4], cosmology has entered a golden era where precise scientific data has become available. Cosmologists are no longer 'often in error, but seldom in doubt' (L D Landau). Cosmology has become a hard science very well worth contributing to. What are the challenges and problems in cosmology where the concepts of macroscopic electromagnetism can become useful?

\section{Current and Future Challenges}

One of the major problems of theoretical physics is the unification of the underlying theory of cosmology and gravity, general relativity, with quantum mechanics. One may wonder whether there are deeper reasons why quantum mechanics and general relativity seem incompatible. Philosophically, quantum mechanics and general relativity belong to different categories: quantum mechanics in its general form is a metatheory concerned with the transition from potentiality to reality, general relativity is the theory of space and time. Quantum mechanics does not exist in real space it exists in Hilbert space, so why should general relativity reside in Hilbert space as well? As the philosophical categories of quantum mechanics and general relativity are different and of equal rank, why

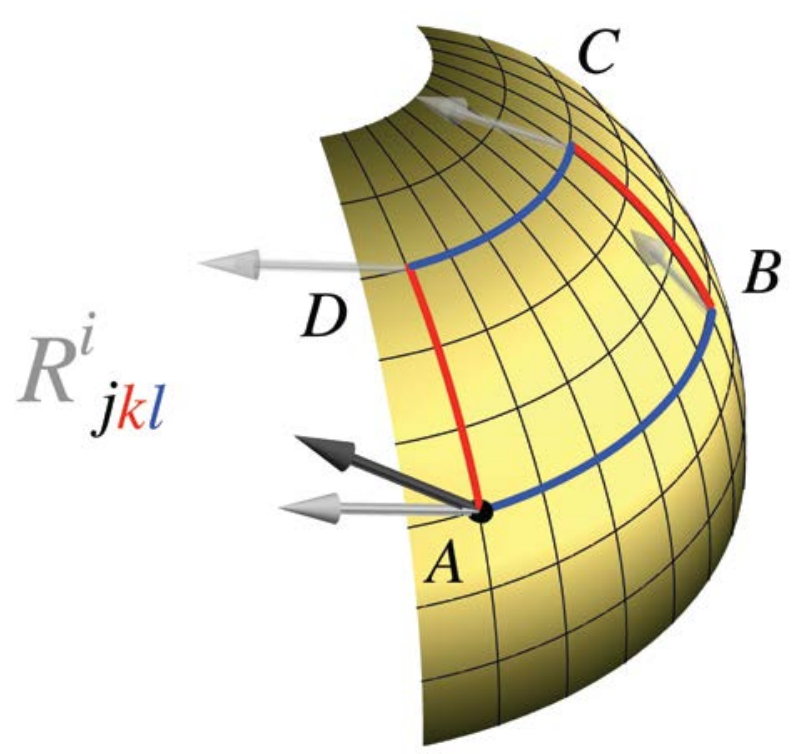

Figure 1 Curved spaces correspond to dielectric media for electromagnetic fields. The figure visualizes Riemann's curvature tensor that quantifies the degree of curvature in differential geometry (reproduced from [1]).

should quantum mechanics reign over general relativity? This is perhaps how a philosopher might argue. A physicist would ask the question what the evidence for quantum physics in general relativity is.

There is no experimental evidence for quantum gravity whatsoever. But is there some theoretical evidence, some hint hidden in the theory that suggests a quantum origin for general relativity? In fact, there is one: the principle of least action. General relativity obeys the action principle - Einstein's field equations follow from Hilbert's action. An action principle is incomprehensible in classical physics: how would, for example, a mechanical particle 'know' in advance that when it starts at point A and ends at point B it should minimize its action along the trajectory? How should space-time 'know' in advance its evolution? Quantum mechanics resolves this puzzle: the mechanical particle takes all paths, the paths interfere with each other with the action as phase, and then the most probable one is the path of minimal action. Similarly, in the quantum picture all space-time geometries should coexist as quantum possibilities, and then their interference singles out the most probable one minimizing Hilbert's action of Einstein's equations. The quantum picture would explain Hilbert's action, even if we have not found the precise picture yet.

In my opinion, the action principle is the strongest argument for quantum gravity. So, if quantum gravity does not exist, what would replace the principle? Is there an alternative, equally fundamental derivation of Einstein's equations? Yes there is: Jacobson has found that Einstein's equations follow from thermodynamics 
as an equation of state [5]. For this, Jacobson considered a causal horizon (a light cone) and associated an entropy with the horizon area and a temperature. These ideas came from Bekenstein's concept of black-hole thermodynamics [6]. Jacobson has generalized them from the horizons of black holes to arbitrary causal horizons. There, the role of the temperature plays the Unruh temperature [7] seen by an accelerated observer that probes the causal horizon. Yet the Unruh temperature is still a concept derived from quantum mechanics. Moreover, the Unruh radiation of accelerated observers has never been observed in an experiment. There, I believe, is room for insights and techniques from macroscopic electromagnetism and optics.

Another major puzzle of cosmology is the observed fact [4] that the universe is accelerating at a rate far exceeding its visible matter content (Fig. 2). Some form of 'dark energy' - for want of a better term with repulsive gravity seems to contribute to the lion's share of the expansion, accounting for about $70 \%$ of the universe's mass. Additionally, about a quarter of the mass appears to be made up by 'dark matter'; only some $5 \%$ of the matter of the universe is of known physics (Fig. 2). Some form of 'dark energy' of a different strength may also have driven the observed rapid inflation of the universe shortly after the big bang. It was speculated that the 'dark energy' originates from the fluctuation energy of the quantum vacuum in the universe. However, a simple estimation of this energy, using a cut-off at the Planck scale, gives a result that disagrees with the observed rate by 120 orders of magnitude [8]. Yet ideas of cosmology in the laboratory have already come to the rescue of the theory: Volovik [9] developed a thermodynamical argument - based on the analogy of cosmology with the physics of superfluid Helium - that the 'dark energy' should be close to zero. The actual value is indeed close to zero, but not quite. It remains to calculate the finite part. Apart from Volovik's idea [9], not much progress has been made in solving this mystery.

\section{Advances in Science and Technology to Meet Challenges}

I believe that experimental techniques from quantum optics will progress to the stage where an observation of the Unruh effect becomes possible. Such techniques are based in the analogy between moving media and space-time geometries [1]. With manipulations of light fields one could create the accelerations required. Such experiments will clarify the physical essence of the Unruh effect and hence give a better justification of Jacobson's thermodynamical argument for the Einstein equation as an equation of state.
I also believe that advances in both theory and experiment of the forces of the quantum vacuum [10]

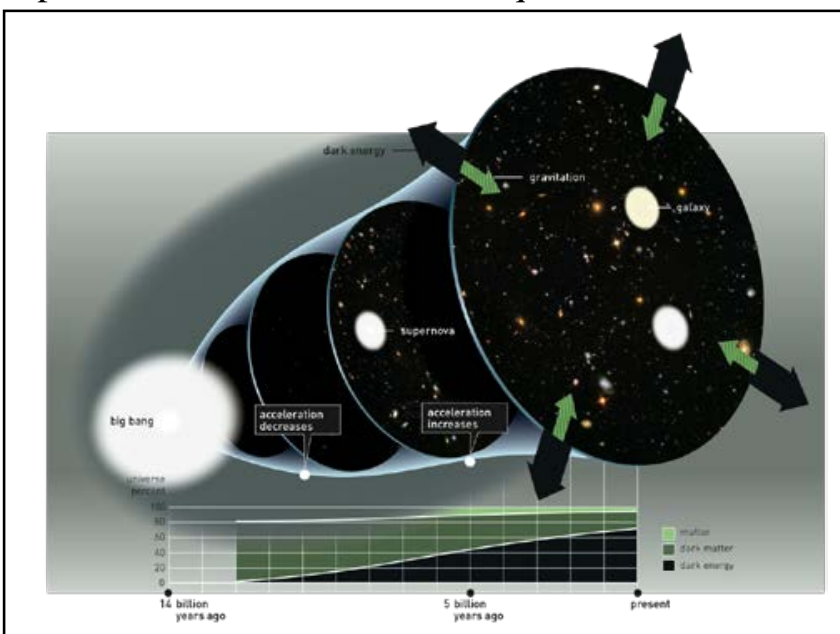

Figure 2. Expansion of the universe. The observed expansion is dominated by unobserved dark energy (black) and dark matter (olive green). Both represent a mystery, in particular dark energy, that appears to generate repulsive gravity. Figure reproduced from https://www.nobelprize.org/ popular material to the 2011 Nobel Prize in physics.

will shed some light on 'dark energy'. There we have theoretical tools available that are testable in modern high-precision experiments and may thus have a basis in fact. Using the analogy between vacuum forces in media and vacuum forces in space-time geometries we can then extrapolate this known laboratory physics to the physics of space. Whether this idea gives the right order of magnitude for the expansion is not clear yet, but at the very least we will know whether the quantum vacuum can be made responsible - or not - for the expansion of the universe.

\section{Concluding Remarks}

Transformation optics has been inspired by the analogy between electromagnetism in media and in the spacetime of general relativity [1]; it is time to see whether macroscopic electromagnetism and optics can make conceptual contributions to cosmology. We discussed two possible areas where this might happen: the Unruh effect and quantum forces in cosmology. There transformation optics has the potential to contribute to fundamental science.

\section{Acknowledgments}

I thank Yael Avni, David Bermudez, Yehonathan Drori, Mathias Fink, Emmanuel Fort, Itay Griniasty, Mordehai Milgrom, Sahar Sahebdivan, Ephraim Shahmoon, William Simpson, and Yana Zilberg for stimulating discussions. This work is supported by the European Research Council and the Israel Science Foundation, a research grant from Mr. and Mrs. Louis 
2

3

Rosenmayer and from Mr. and Mrs. James Nathan, and the Murray B. Koffler Professorial Chair.

\section{References}

[1] Leonhardt U and Philbin T 2010 Geometry and Light: The Science of Invisibility (Dover, Mineola)

[2] Plebanski J 1960 Electromagnetic Waves in Gravitational Fields Phys. Rev. 118 1396-1408

[3] Abbott B P et al. 2016 Observation of Gravitational Waves from a Binary Black Hole Merger Phys. Rev. Lett. 116061102

[4] Perlmutter S, Smith B P and Riess A G 2012 Nobel lectures Rev. Mod. Phys. 84 1127-1175

[5] Jacobson T 1995 Thermodynamics of Spacetime: The Einstein Equation of State Phys. Rev. Lett. 1260-1263

[6] Bekenstein J D 1974 Generalized second law of thermodynamics in black-hole physics Phys. Rev. D 9 3292-3300

[7] Unruh W G 1976 Notes on black-hole evaporation Phys. Rev. D 14 870-892

[8] Brumfiel G 2007 Unseen Universe: A constant problem Nature 448, 245-248

[9] Volovik G E 2003 The Universe in A Helium Droplet (Clarendon Press, Oxford)

[10] Simpson W M R and Leonhardt U editors 2015 Forces of the Quantum Vacuum (World Scientific, Singapore) 
8.2 Spacetime analogs based on hyperbolic metamaterials Igor I. Smolyaninov ${ }^{1}$ and Vera N. Smolyaninova ${ }^{2}$

${ }^{1}$ University of Maryland

${ }^{2}$ Towson University

\section{Status}

Modern developments in gravitation research indicate that classic general relativity is an effective macroscopic theory, which needs to be replaced with a more fundamental description based on yet unknown microscopic degrees of freedom. However, our ability to obtain experimental insights into the future fundamental theory is strongly limited by low energy scales available to terrestrial physics. The emergent analogue spacetime program offers a promising way around this difficulty. Looking at such systems as superfluid helium and atomic Bose-Einstein condensates, physicists discover how macroscopic field theories arise from well-studied atomic degrees of freedom. Recent introduction of metamaterials and transformation optics appear to be an exciting new development in this field. Metamaterial optics is not limited by the properties of atoms and molecules given to us by nature. "Artificial atoms" used as building blocks in metamaterial design offer much more freedom in constructing analogues of various exotic spacetime metrics, such as black holes [1], wormholes [2], cosmic strings [3], and even the metric of the Big Bang [4]. Explosive development of this field promises new insights into the fabric of spacetime, which cannot be gleaned from any other terrestrial experiments. In addition, compared to the standard general relativity, metamaterial optics gives more freedom to design an effective space-time with very unusual properties. Light propagation in all static general relativity situations can be mimicked with positive $\varepsilon_{i k}=\mu_{i k}$ [5], while the allowed parameter space of the metamaterial optics is broader. Thus, flat Minkowski space-time with the usual $(-,+,+,+)$ signature does not need to be a starting point. Other effective signatures, such as the "two times" physics $(-,-,+,+)$ signature may be realized [6]. Metric signature change events (in which a phase transition occurs between say $(-,+,+,+)$ and $(-,-,+,+)$ space-time signature) are being studied in BoseEinstein condensates and in some modified gravitation theories (see ref.[7], and the references therein). It is predicted that a quantum field theory residing on a spacetime undergoing a signature change reacts violently to the imposition of the signature change. Both the total number and the total energy of the particles generated in a signature change event are formally infinite. While optics of bulk hyperbolic metamaterials provides us with ample opportunities to observe metric signature transitions [6], even more interesting physics arise at the metamaterial interfaces. Very recently it was demonstrated that mapping of
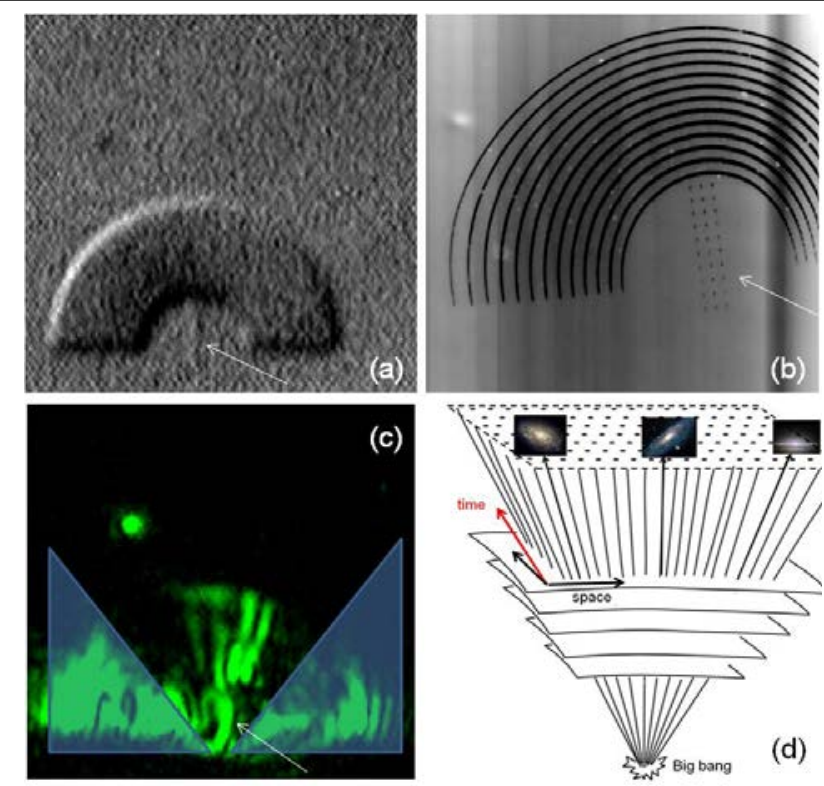

toy "big bang"

(d)

Figure 1 Experimental model of world line behaviour in an "expanding universe" using a plasmonic hyperbolic metamaterial [4]: Optical (a) and AFM (b) images of the metamaterial. The plasmon source is shown by arrows. (c) Plasmonic rays or "world lines" increase their spatial separation as a function of "timelike" radial coordinate. For the sake of clarity, edge scattering is partially blocked. (d) Schematic view of world lines behaviour near the Big Bang.

monochromatic extraordinary light distribution in a hyperbolic metamaterial along some spatial direction may model the "flow of time" in a three dimensional $(2+1)$ effective Minkowski spacetime [4]. If an interface between two metamaterials is engineered so that the effective metric changes signature across the interface, two possibilities may arise. If the interface is perpendicular to the time-like direction $z$, this coordinate does not behave as a "timelike" variable any more, and the continuous "flow of time" is interrupted. This situation (which cannot be realized in classic general relativity) may be called the "end of time". It appears that optics of metamaterials near the "end of time" event is quite interesting and deserves a detailed study. For example, in the lossless approximation all the possible "end of time" scenarios lead to field divergencies, which indicate quite interesting linear and nonlinear optics behaviour near the "end of time". On the other hand, if the metamaterial interface is perpendicular to the spacelike direction of the effective $(2+1)$ Minkowski spacetime, a Rindler horizon may be observed (Rindler metric approximates spacetime behaviour near the black hole event horizon [8]).

\section{Current and Future Challenges}

Experimental realization of various metamaterial spacetime analogs described above requires engineering of low loss hyperbolic metamaterials 
having spatially-dependent uniaxial anisotropic dielectric permittivity tensors with opposite signs of their diagonal components $\varepsilon_{\mathrm{x}}=\varepsilon_{\mathrm{y}}=\varepsilon_{1}>0$ and $\varepsilon_{\mathrm{z}}=\varepsilon_{2}<0$. The wave equation, which describes propagation of extraordinary light in such metamaterials is formally equivalent to a $3 \mathrm{D}$ Klein-Gordon equation describing a massive scalar field $\varphi_{\omega}$ :

$$
-\frac{\partial^{2} \varphi_{\omega}}{\varepsilon_{1} \partial z^{2}}+\frac{1}{\left|\varepsilon_{2}\right|}\left(\frac{\partial^{2} \varphi_{\omega}}{\partial x^{2}}+\frac{\partial^{2} \varphi_{\omega}}{\partial y^{2}}\right)=\frac{\omega_{0}^{2}}{c^{2}} \varphi_{\omega}=\frac{m^{* 2} c^{2}}{\hbar^{2}} \varphi_{\omega}
$$

in which the spatial coordinate $\mathrm{z}=\tau$ behaves as a "timelike" variable, and $\varepsilon_{z}=\varepsilon_{2}$ plays the role of a timedependent scale factor. Therefore, eq.(1) describes world lines of massive particles which propagate in a flat $(2+1)$ Minkowski spacetime. When a metamaterial is built and illuminated with a coherent extraordinary CW laser beam, the stationary pattern of light propagation inside the metamaterial represents a complete "history" of a toy $(2+1)$ dimensional spacetime populated with particles of mass $\mathrm{m}^{*}$. This "history" is written as a collection of particle world lines along the "timelike" $\mathrm{z}$ coordinate, as illustrated in Fig.1. While such linear "static" models are interesting to fabricate and study, it is clear that non-linear "dynamic" self-assembled hyperbolic metamaterial systems in which the effective spacetime configuration is defined by temperature, external fields and various physical interactions of the constituent parts will provide a much more interesting playground for the emergent analogue spacetime paradigm.

\section{Advances in Science and Technology to Meet Challenges}

The latter challenge has been met in recent studies of ferrofluid-based self-assembled hyperbolic metamaterials [9]. Such fluid-based metamaterials exhibit strong nonlinearities, so that nonlinear light propagation through the ferrofluid may be described in a similar fashion as in general relativity. Moreover, when the ferrofluid is subjected to a modest external magnetic field, the nanoparticles inside the ferrofluid form small hyperbolic metamaterial domains (as illustrated in Fig.2), which from the electromagnetic standpoint behave as individual "Minkowski universes" inside a Euclidean background: the metric signature transition in a ferrofluid leads to separation of the effective spacetime into a multitude of intermingled Minkowski and Euclidean domains, giving rise to a picture of "metamaterial multiverse" [9]. As illustrated in Fig.2, inflation-like behaviour appears to be generic within the individual Minkowski domains. Thus, ferrofluid-based self-assembled metamaterial geometry captures many features of several cosmological models of the multiverse, such as metric signature transition scenario in loop quantum

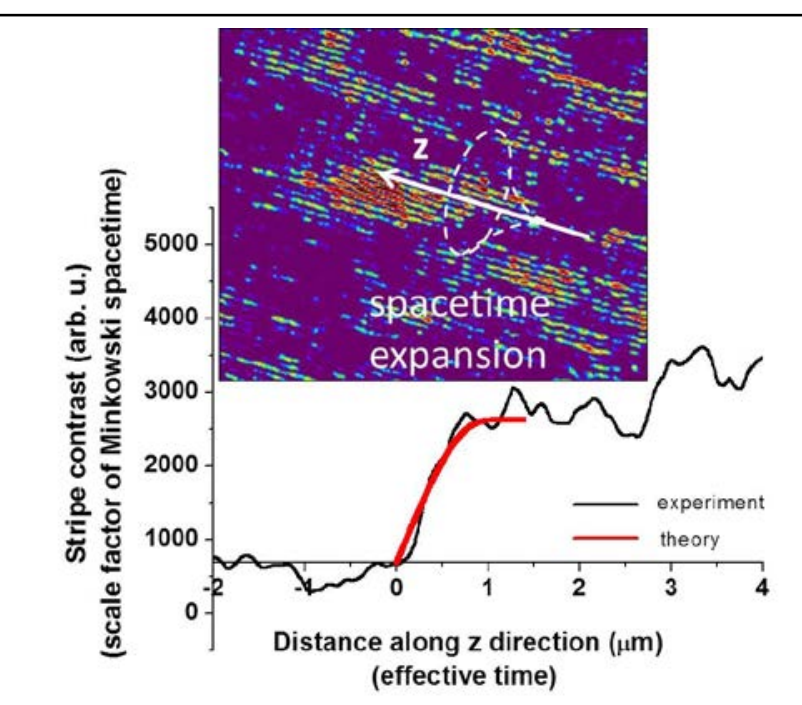

Figure 2 Magnified image of one of the Minkowski domains in a ferrofluid illustrates inflation-like expansion of the effective spacetime near the domain wall. The plot compares measured and theoretically calculated dependencies of the spacetime scale factor $-\varepsilon_{\mathrm{z}}$ on the effective time. The calculations are based on analysis of the measured stripe contrast in the image

cosmology [7], natural emergence of a large number of Minkowski universes, and inflation. Moreover, it also appears that due to variations in size and local magnetic field, individual Minkowski domains in the ferrofluid exhibit different "laws of physics", such as different strength of effective gravity, and different radiation lifetimes due to variations in the local Purcell factor. Thus the ferrofluid-based model may be used to illustrate the fine-tuning mechanism in cosmology. It is remarkable that all these effects may be studied via direct microscopic observations, as illustrated in Fig.2. In addition, the ferrofluid-based macroscopic selfassembled 3D metamaterials may also exhibit reach physics associated with microscopic topological defects of the effective Minkowski spacetime. As was pointed out recently by Mielczarek and Bojowald [10], the properties of self-assembled magnetic nanoparticlebased hyperbolic metamaterials exhibit strong similarities with the properties of some microscopic quantum gravity models, such as loop quantum cosmology.

\section{Concluding Remarks}

Despite these very interesting features of the hyperbolic metamaterial-based spacetime models, we should emphasize that the described analogy between the extraordinary light propagation inside the ferrofluid and the dynamics of massive particles in Minkowski spacetime is far from being perfect. The main difficulty comes from the cross-coupling between extraordinary and ordinary light inside the ferrofluid, which may be caused by domain interfaces and internal defects. Since ordinary light does not obey the same wave equation (1), such a cross-coupling breaks the effective Lorentz 
symmetry of the system. In addition, such a model is necessarily limited to $2+1$ spacetime dimensions. Nevertheless, despite these limitations the developed metamaterial model of the cosmological multiverse appears to be quite interesting, since it is able to replicate many of its hypothesized features in the laboratory setting.

\section{References}

[1] Smolyaninov I 2003 Surface plasmon toy-model of a rotating black hole New Journal of Physics 5, 147

[2] Greenleaf A, Kurylev Y, Lassas M, Uhlmann G 2007 Electromagnetic wormholes and virtual magnetic monopoles from metamaterials Phys. Rev. Lett. 99, 183901

[3] Mackay T, Lakhtakia A 2010 Towards a metamaterial simulation of a spinning cosmic string Phys. Lett. A 374, 2305-2308

[4] Smolyaninov I, Hung Y 2011 Modeling of time with metamaterials JOSA B 28, 1591-1595

[5] Landau L, Lifshitz E The Classical Theory of Fields (Elsevier, Oxford 2000).

[6] Smolyaninov I, Narimanov E. 2010 Metric signature transitions in optical metamaterials Phys.Rev. Lett. 105, 067402

[7] White A, Weinfurtner S, Visser M 2010 Signature change events: A challenge for quantum gravity? Class. Quantum Gravity 27, 045007

[8] Smolyaninov I, Hwang E, Narimanov E 2012 Hyperbolic metamaterial interfaces: Hawking radiation from Rindler horizons and spacetime signature transtions Phys. Rev. B 85, 235122

[9] Smolyaninov I, Yost B, Bates E, Smolyaninova V 2013 Experimental demonstration of metamaterial "multiverse" in a ferrofluid Optics Express 21, 14918-14925

[10] Bojowald M, Mielczarek J, 2015 Some implications of signature-change in cosmological models of loop quantum gravity J. Cosmology Astroparticle Phys. 08052 


\author{
8.3 Transformation optics in general relativity - \\ Robert T. Thompson \\ Institute of Applied Physics, Karlsruhe Institute of \\ Technology
}

\section{Status}

The similarity of the light-bending property of curved vacuum spacetimes to that of refractive media was one of the earliest predictions of general relativity. Although Eddington's observation of the deflection of starlight passing near the sun confirmed this prediction, he also remarked that the same effect could be achieved in otherwise flat space if the region around the sun were filled with an appropriately refracting medium [1]. Gordon then reversed the argument by asking whether a refracting medium could be identified with a curved spacetime, and found the optical metric of an isotropic medium residing in a curved background spacetime [2]. While studying the propagation of electromagnetic waves in gravitational fields, Plebanski obtained

$\varepsilon=\mu=-\frac{\sqrt{-g}}{g_{00}} g^{i j}, \gamma=-\epsilon_{i j k} \frac{g_{0 j}}{g_{00}}$

as the effective constitutive relations for curved spacetimes [3].

Since the actual relative permeability and permittivity of free space are always identically $\varepsilon=\mu=1$, with $\gamma=0$, Plebanski's result should be understood as identifying curved spacetime solutions of Maxwell's equations with solutions inside a refracting medium in otherwise flat spacetime, thereby mimicking in some way the lightdeflecting properties of the vacuum. This recipe for a laboratory-accessible dielectric analog representation of a curved spacetime was exploited by de Felice to describe the dielectric analog of a black hole [4], residing in flat spacetime.

The geometric, or spacetime, picture of transformation optics (TO) established by Leonhardt and Philbin [5] turns out to take the same form as Eqs. (1), but arises from a slightly different conceptual basis than analog spacetimes.

Both can be described in terms of diffeomorphisms between manifolds, but whereas analog spacetimes are a projection from curved to flat spacetimes [6], TO maps a given spacetime to itself [5,7]. For TO in flat spacetime, one finds the transformation medium to be given by Eqs. (1), but where one uses the transformed metric

$g_{\mu \prime v^{\prime}}=\Lambda_{\mu \prime}^{\mu} \Lambda_{v^{\prime}}^{v} g_{\mu \nu \cdot(2)}$

\section{Current and Future Challenges}

The transformations involved in TO are linear operations that preserve the symmetry of the initial configuration. The dispersionless, impedance-matched vacuum is transformed to dispersionless, impedancematched media, and we cannot expect to generate nonlinearities from linear processes. Thus, as it stands, TO is not very good at modelling or controlling these real-world phenomena.

Progress in TO, and the ability to draw upon ideas from general relativity and apply them to optics, is predicated on 1) advancing the spacetime-covariant, tensorial formulation of electrodynamics in media that is compatible with the curved manifolds of relativity, and 2) expanding the transformation concept to control these advanced features.

Progress to date has been achieved through covariant, tensorial descriptions of real-world phenomena in media. For example, recent developments improve the accounting of properties like dispersion and nonlinearities - but the ability to control or engineer them through a transformation procedure is limited.

Utility has been gained by restricting the set of transformations, e.g. conformal or quasi-conformal, but expanding the transformation concept is more challenging. It may be tempting to try generalizing Leonhardt and Philbin's virtual space by interpreting it as a truly curved virtual manifold, thereby defining a "projective spacetimes TO" as an analog spacetimes type projection into a medium living in flat spacetime, but such an approach requires caution.

For analog spacetimes, Eq. (1) stems from a particular choice of projection map between curved and flat spacetimes (one with Jacobian matrix $\Lambda=I$ ) [6]. But there is no natural identification of curved spacetime with a medium in flat spacetime, and thus no canonical choice of projection exists. As a result, analog spacetimes are manifestly non-covariant since 1 ) any spacetime can be represented by an infinite number of physically inequivalent media, which must be interpreted relative to the chosen projection, and 2) two different coordinate representations of the same curved vacuum do not correspond to two different coordinate representations of the same analog [8].

Furthermore, this type of projection connects nonisometric spaces and therefore the medium cannot simultaneously mimic all aspects of light propagation in the curved spacetime, in much the same way that any flat map of Earth always introduces distortions to some surface features [9]. The Plebanski map preserves some idea of the coordinate description of a ray trajectory, but it does not simultaneously preserve physically meaningful and measurable quantities like the evolution of the cross sectional area of a beam, i.e. the focus, instead distorting these features [9]. In other words, given projection $\varphi$, observers in each manifold, making measurements on $\varphi$-related congruences with their own 
respective metrics, will report different results for some measurements.

\section{Advances in Science and Technology to Meet Challenges}

Despite both the longstanding, and recent TO-inspired, interest in the analogy between spacetimes and refracting media, relatively little has been done on the covariant, tensorial formulation of electrodynamics in refracting media within a curved background spacetime.

Early work by Post established a tensorial formulation of electrodynamics in media [10], but the crucial aspect lacking from Post's analysis is a distinction between the spacetime and medium contributions. Such an extension may be made for linear media in an arbitrary spacetime by writing Maxwell's equations as [7]

$d F=0, d G=J, G=* \chi F(3)$

where the Hodge star * contains all information about the background spacetime, while the tensor $\chi$, whose vacuum value $\chi_{\text {vac }}$ is uniquely defined such that the vacuum is a trivial dielectric, incorporates complete information about the linear medium, i.e. permeability, permittivity, and magnetoelectric couplings.

By explicitly separating contributions of the spacetime and the medium, TO is understood as an automorphism of the manifold, where the spacetime metric and coordinates are fixed once and for all and the transformation acts directly on the fields [7]. This formulation explicitly recognizes the unalterability of the background spacetime, which need not be flat, and the transformation has a physical interpretation as the insertion of $\chi$ into the system. It has also been shown that this formulation is fully covariant [8], and since it is isometric by construction it does not the suffer the infidelity of "projective spacetimes TO."

Inside refracting media, the lightcone does not coincide with that of the background spacetime, and light is in general both non-geodesic and non-null with respect to the background spacetime. Instead, the lightcone is defined by an emergent structure called the optical metric that contains both background metric and medium contributions. Physically, an observer makes measurements with respect to the background spacetime metric, so the optical metric should be understood as an additional structure rather than a replacement of the background metric.

Since the optical metric controls many aspects of light propagation, a better understanding of it could lead to improvements in the construction of transformation media. A fully covariant, tensorial expression for the optical metric of a general linear medium in an arbitrary spacetime, that distinguishes medium and spacetime contributions, does not currently exist. Obtaining such an expression would enhance our understanding of light in media.

In the geometric optics limit, first and second order kinematics of congruences can provide generalizations of the Raychaudhuri and geodesic devaition equations in terms of the optical metric - standard tools in general relativity that describe the evolution of congruences that will enhance analysis and ray tracing of beams in media.

At the next order of expansion beyond ray optics, the transport equations provide information on the evolution of the polarization, and chirality. A fully covariant formulation of the transport equations in media would enable a rigorous understanding of how these features behave in TO, and may result in a methodology that allows control independently of the ray trajectory.

Impedance matching is used as a quantifier of the "no reflection" concept and has clear relevance to TO, but it is so far unclear how this arises in the context of spacetime covariant electrodynamics in media.

The inclusion of dispersion in TO is discussed in section 7.2, and has primarily been achieved by adopting a differential operator approach [11]. An integral kernel approach is potentially more general, but so far has only been studied by considering the simplified case where the propagator is assumed to be that of a background Minkowski spacetime, which neglects both the possibility of a curved background spacetime and the modification to the propagator concordant with the modified lightcone within the medium. Ultimately, an understanding of the covariant Green tensor in media within curved spacetimes is necessary for a full-wave analysis and scattering matrix calculations. At this level, electromagnetic waves can reflect off the spacetime curvature and self-interfere - important effects that are not currently modeled in conjunction with ordinary refraction in flat spacetimes.

\section{Concluding Remarks}

By developing a fully spacetime covariant, tensorial theory for electrodynamics in media within curved spacetimes, conceptual issues can be resolved and new features can be included in TO. A few of the possible next steps in this programme have been outlined here.

Ideally, we should eventually be able to discuss all of classical optics in media within a fully spacetimecovariant formalism, which could have applications beyond TO. For example, almost everything we know about the universe comes from observations of electromagnetic waves, and curvature effects like gravitational lensing provide important information. But the universe is not empty; light also propagates through potentially refractive dust, gas clouds, and 
accretion disks around massive objects, the proper accounting of which could shed additional light on the universe.

\section{References (separate from the two page limit)}

[1] A.S. Eddington, Space, Time, and Gravitation, Cambridge, University Press (1920).

[2] W. Gordon, "Zur Lichtfortpflanzung nach der Relativitätstheorie,” Annalen der Physik 72421 (1923).

[3] J. Plebanski, "Electromagnetic waves in gravitational fields,” Physical Review 1181396 (1960).

[4] F. de Felice, "On the gravitational field acting as an optical medium," General Relativity and Gravitation 2347 (1971).

[5] U. Leonhardt and T.G. Philbin, "General relativity in electrical engineering," New Journal of Physics 8247 (2006).

[6] R.T. Thompson and J. Frauendiener "Dielectric analog spacetimes," Physical Review D 82124021 (2010).

[7] R.T. Thompson, S.A. Cummer, and J. Frauendiener, "A completely covariant approach to transformation optics,” Journal of Optics 13024008 (2011).

[8] R.T. Thompson and M. Fathi, "Shrinking cloaks in expanding space-times: The role of coordinates and the meaning of transformations in transformation optics,” Physical Review A 92 013834 (2015).

[9] M. Fathi and R.T. Thompson, "Cartographic distortions make dielectric spacetime analog models imperfect mimickers," Physical Review D 93124026 (2016).

[10] E.J. Post, Formal Structure of Electromagnetics, North Holland (1962).

[11] Gratus, et. al. "On spacetime transformation optics: temporal and spatial dispersion," New J. of Physics 18123010 (2016). 


\section{Optics and Beyond}

\subsection{Seeking applications in optics and beyond - Martin Wegener and Muamer Kadic}

Karlsruhe Institute of Technology (KIT)

\section{Status}

We view transformation optics as a design tool - a very intuitive and mathematically intriguing design tool. To design what? The fascinating idea of macroscopic omni-directional broadband free-space invisibility cloaks for electromagnetic waves has served as a catalyzing example. However, it is now clear that fundamental bandwidth restrictions imposed by relativity (i.e., the impossibility of superluminal energy transport) mean that invisible people walking on the streets will forever remain a matter of science fiction.

So where should this field go? What real-world devices can we design? After all, researchers have designed optical devices such as complex lens systems by means other than transformation optics for centuries already. We still see opportunities and challenges in visiblefrequency optics, but even more so in areas beyond optics, for which the above fundamental restrictions due to relativity do not apply.

\section{Current and Future Challenges}

Electrical contacts on optical devices are ubiquitous. Examples include solar cells, photodetectors, and large-area organic light-emitting diodes (OLEDs) as future wall paper. On the one hand, metal contacts on top of these device are wanted electrically, e.g., to avoid losses by serial resistances. On the other hand, they are unwanted optically because the contacts cast shadows, thereby reducing conversion efficiency in solar cells, reducing effective quantum efficiency in detectors, or leading to spatially inhomogeneous light emission in OLED wall paper. Transparent contacts (such as indium-tin oxide films in touch screens) are often a solution, but not always. In these cases, one needs a device on top of the contact that guides the light around the contact.

At first sight, this task sounds just like any ordinary invisibility cloak. While one even wants operation for a broad range of visible colors, these invisible-contact problems are simpler in various ways. One needs to distinguish between the ballistic and the diffusive regime of light propagation. The ballistic regime applies to solar cells or photodetectors, whereas OLEDs are Lambertian light emitters and often diffusive light scattering layers are added on top of them.
Metal contacts on solar cells can cover up to $10 \%$ of the usable area. We have designed and realized threedimensional broadband graded-index cloaks [1] via Schwarz-Christoffel conformal maps [2]. But this approach is overkill because the phase of light or, equivalently, the time of arrival of light plays no role for this application. Furthermore, a solar cell is a deadend street because all the light hitting the active area ideally gets absorbed. These aspects allow for a modified and simpler approach in which we again start from a spatial coordinate transformation (in fact, a 1D version of Pendry's transformation of a point to a circle/sphere). Rather than mapping it onto a materialparameter distribution, we map it onto a dielectric freeform surface, i.e., onto the shape of a surface of a bulk dielectric such as glass or a polymer [2] (see Figure 1).

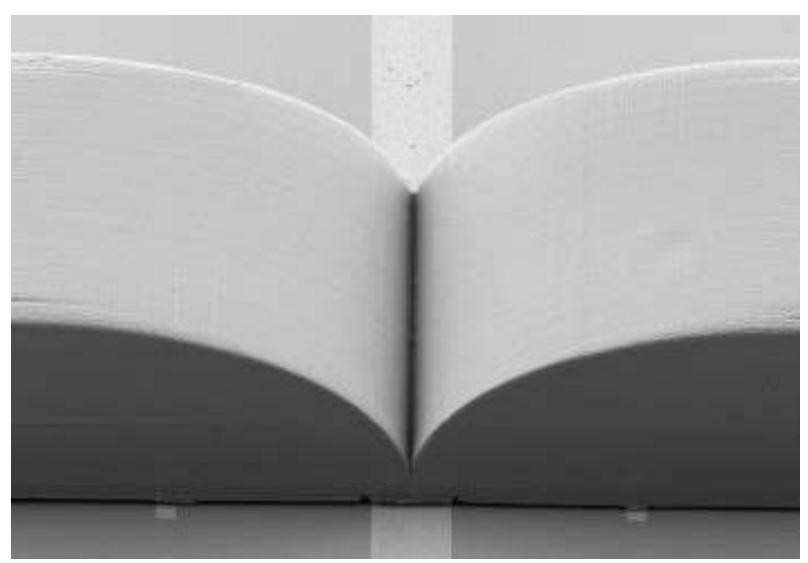

Figure 1 - Electron micrograph of a fabricated cloak for a metal contact (20 $\mu \mathrm{m}$ width) on a silicon surface. The structure has been designed by mapping a $1 \mathrm{D}$ coordinate transformation onto a dielectric (polymer) free-form surface, which refracts the incident light such that it avoids the metal contact. Reproduced with permission of OSA from Reference [2].

For 1D arrays of long metal wires, this approach works amazingly well for all angles, polarizations, and colors of incident sun light, even for area filling fractions of the metal contacts as large as $20 \%$. Furthermore, after making a master by state-of-the-art 3D laser nanolithography, the resulting structures can be massreplicated by established imprinting techniques, such that real-world applications in terms of solar cells on your roof appear possible [3]. Many solar cells, however, do have additional wider bus bar lines orthogonal to the metal fingers discussed. This more demanding cloaking problem has not been solved yet.

The cloaking of metal contacts on OLEDs is a distinct problem. In other words: If you just use a free-form surface like above, designed for a solar cell, it will not work [4]; it will even make the shadow cast by the OLED contact more pronounced. The reason lies in the different angular distributions of light rays. Nevertheless, one can use a different spatial transformation that leads to a different free-form 
surface. This spatial transformation, however, turns out to be an implicit one; the spatial transformation depends on the result, requiring an iterative solution. While our corresponding results are encouraging (unpublished), we emphasize that again only the 1D problem of isolated wires or arrays of parallel metal wires has been solved. The problem of cloaking hexagonal or square lattices of contact wires, remains open.

As an alternative, we have shown that the idea of cloaking based on the macroscopic Maxwell equations can be translated to the regime of light diffusion, essentially because the stationary version of Fick's diffusion equation is mathematically analogous to electrostatics [4-7]. Here, the light diffusivity is controlled by the density of scattering particles. On this basis, we have successfully cloaked contacts [4]. The remaining challenge lies in the 3D micromanufacturing of such structures in a manner suitable for mass products. Again, the problem of hexagonal or square lattices of contact wires has not been solved so far.

Together with the above free-form surface approach, in regard to metal contacts on Lambertian OLEDs, the two above possibilities compete against each other, while we are presently not aware of other competing approaches.

Let us consider an example from mechanics. In civil engineering, one often needs some sort of light-weight scaffold structure. Suppose you need to punch a hole into this structure, e.g., for obtaining a feedthrough. Obviously, this hole will weaken the support structure, raising the question whether we can build something around the void to make the overall arrangement appear in all respects as without the hole being punched in. In other words: We aim at a mechanical cloak. In 2006, Milton showed that the continuummechanics elasticity equations are not form-invariant under general spatial transformations, neither in the static nor in the dynamic case (which is a show stopper for the ideas of transformation optics) - at least not for the elasticity tensors of ordinary materials. In selected special cases, such as 2D flexural waves one gets away with ordinary solids [8] or for 3D pressure waves with pentamode mechanical metamaterials [9]. The latter can be seen as solids approximating liquids in that their shear modulus is small compared to their bulk modulus.

Another option in mechanics (and in other areas) is to use direct spatial transformations of discrete lattices [10]. This simple and direct approach works amazingly well in two dimensions and in the static case (also see Figure 2), but so far lacks a sound theoretical justification in regard to why it also takes care of the shear forces. Another challenge is to extend this approach to the wave regime.

Yet another avenue is based on the fact that the elasticity equations do become form invariant for a more general class of elastic solid, e.g., for so-called Cosserat materials, which exhibit additional (rotational) degrees of freedom. One opportunity and challenge of the field lies in designing and experimentally realizing such generalized elastic solids in microstructured form to then use them for general transformation mechanics.

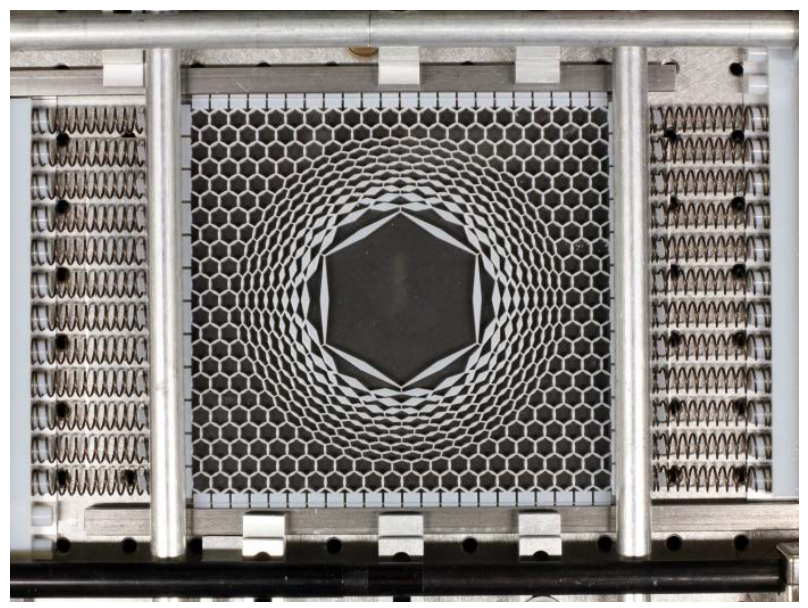

Figure 2 - Photograph of a fabricated and characterized twodimensional static mechanical cloak, designed by using the direct-lattice-transformation approach. The lattice constant of the hexagonal lattice in the surrounding of the cloak is about $a=7 \mathrm{~mm}$. Reproduced with permission from Reference [10].

\section{Advances in Science and Technology to Meet Challenges}

Generally, approaches solving the inverse problem of how to get from the material parameters derived from transformation optics (or counterparts thereof) to concrete metamaterial microstructures need to be developed.

In regard to cloaking of general elastic waves by microstructured cloaks (compare Figure 2), perfectly matched layers (PMLs) at simulation domain boundaries will need to be worked out - otherwise such cloaks cannot even be tested numerically on the computer.

The corresponding fabrication technologies are almost there. However, the field would certainly benefit from advances in 3D laser nanoprinting in regard to speed, spatial resolution, precision, and from the availability of a wider range of ingredient materials.

\section{Concluding Remarks}

Real-world applications of transformation optics and invisibility cloaking such as cloaked contacts on solar cells or on OLEDs have come into reach. Further 
application possibilities arise beyond optics, e.g., in mechanics. There, however, much further basic work needs to be done.

Acknowledgments - We acknowledge the contributions of all of the members of the KIT group involved in this work. We also acknowledge support by the Helmholtz program Science and Technology of Nanosystems (STN), by Deutsche Forschungsgemeinschaft (DFG) through program DFG SPP 1839 "Tailored Disorder", and by the Hector Fellow Academy.

\section{References}

[1] Ergin T, Stenger N, Brenner P, Pendry JB, and Wegener M 2010 Three-Dimensional Invisibility Cloak at Optical Wavelengths Science 328, 337-339

[2] Schumann MF, Wiesendanger S, Goldschmidt JC, Bläsi B, Bittkau K, Paetzold UW, Sprafke A, Wehrspohn R, Rockstuhl C, and Wegener M 2015 Cloaked contact grids on solar cells by coordinate transformations: Designs and prototypes Optica 2, 850-853

[3] Schumann MF, Langenhorst M, Ding K, Paetzold UW, and Wegener M 2017 All-angle invisibility cloaking of contact fingers on solar cells by refractive free-form surfaces Adv. Opt. Mater., submitted

[4] Mayer F, Schittny R, Egel A, Niemeyer A, Preinfalk J, Lemmer U, and Wegener M 2016 Cloaking contacts on large-area organic light-emitting diodes Adv. Opt. Mater. 4, 740-745

[5] Schittny R, Kadic M, Bückmann T, and Wegener M 2014 Invisibility Cloaking in a Diffusive Light Scattering Medium Science 345, 427-429

[6] Schittny R, Niemeyer A, Kadic M, Bückmann T, Naber A, and Wegener M 2015 Diffuse-light allsolid-state invisibility cloak Opt. Lett. 40, 42024205

[7] Schittny R, Niemeyer A, Mayer F, Naber A, Kadic M, and Wegener M 2016 Invisibility cloaking in light scattering media Laser Photon. Rev. 10, 382408

[8] Stenger N, Wilhelm M, and Wegener M 2012 Experiments on elastic cloaking in thin plates Phys. Rev. Lett. 108, 014301

[9] Bückmann T, Thiel M, Kadic M, Schittny R, and Wegener M 2014 An elasto-mechanical unfeelability cloak made of pentamode metamaterials Nat. Commun. 5, 4130

[10] Bückmann T, Kadic M, Schittny R, and Wegener M 2015 Cloak design by direct lattice transformation Proc. Natl. Acad. Sci. USA 112, 4930-4934 
9.2 Beyond Optics: Transforming Other Wave And Transport Systems - Steven A. Cummer, Duke University

\section{Status}

Transformation optics was an astonishing theoretical breakthrough. Beyond bringing invisibility into the realm of the feasible, it remains one of few general tools for solving the electromagnetic design and synthesis problem. One can define a particular geometric operation-such as stretching, twisting, and displacing - to be performed on electromagnetic fields, and the transformation optics framework provides exact material parameters to implement that operation.

Following this important discovery, it was naturally of great interest (and importance) to know whether the coordinate transformation framework could be applied to control fields in other wave and transport systems. The peculiarities of coordinate transformations in electromagnetics, embodied in relativity theory, suggested that the answer might be no. However, it was soon shown that the transformation framework could be applied to acoustic waves in fluids without steady flow $[1,2]$. It soon became clear that the concept could be applied to other linear wave and transport systems, including the Schrodinger equation [3] and heat conduction and diffusion [4]. It should be mentioned that the existence of transformation solutions to charge transport had been discovered previously [5].

The ability to control transport and wave propagation in nearly arbitrary ways in a wide range of physical systems offers amazing possibilities for both exploring wave behavior in complex environments and developing devices of practical value. The key step in transitioning transformation theory to physical implementation in these systems is the ability to create the medium effective properties needed to control the transport or wave propagation in each system. In acoustics, for examples, these properties are mass density and bulk modulus (compressional stiffness), while in heat conduction, these are the specific heat and thermal conductivity. Understanding the theoretical capabilities and limits of the transformation approach in each of these physical systems, developing new approaches to control the essential material properties effectively and efficiently, and transferring knowledge and results from one physical system to another all remain active research areas with critical unanswered questions.

\section{Current and Future Challenges}

The applicability of the transformation design approach to at least some non-electromagnetic wave and transport systems has opened the door to new research directions and raises some important scientific questions. One

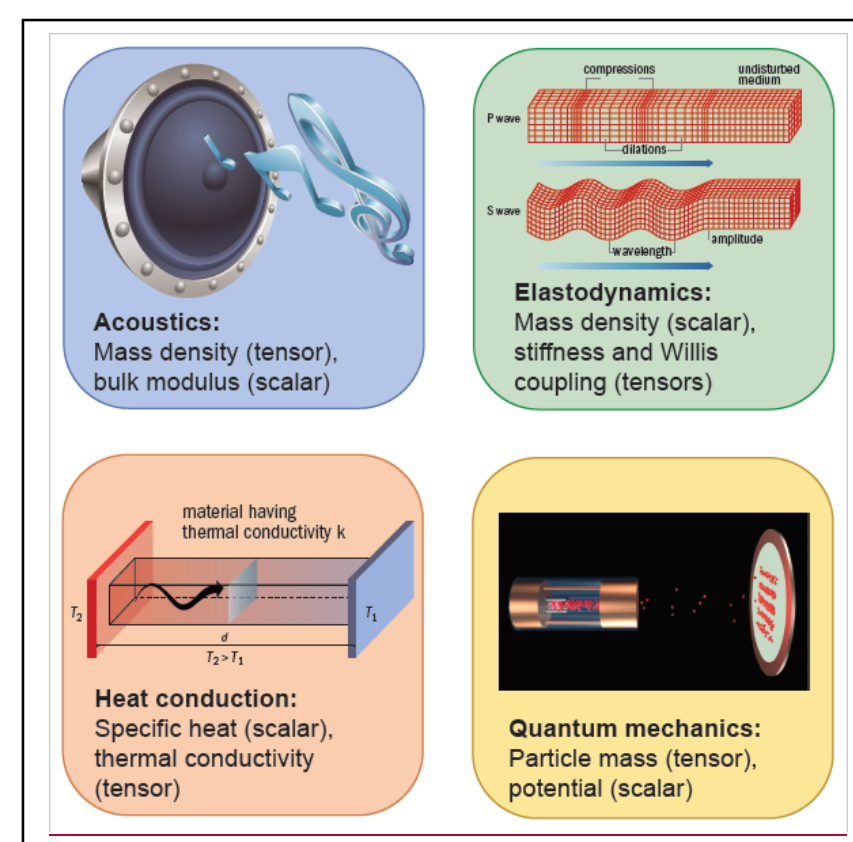

Figure 1 - Illustration of some of the wide range of physical systems that admit transformation-type solutions and the effective material parameters that must be controlled in each case.

question is obvious: are there more systems that can be controlled using the transformation approach, and what are the limits of applicability? This is a largely theoretical question that is waiting to be tackled, should additional wave and transport systems of interest be identified. Past work in this area has followed one of two general approaches: identifying a mapping of the system in question to one known to admit transformation-type solutions, or the demonstration of the coordinate transformation invariance of the dynamic equations themselves.

One research direction enabled by transformation design is the experimental implementation of interesting wave and transport physics by analogue. The similarity of the transformation approach across different wave systems creates connections that enable experiments in one domain to have much broader implications. One example is exploiting the theoretical analogy between light propagation near a black hole and wave propagation in a moving fluid to explore difficult-toaccess phenomena such as Hawking radiation [6, and references therein]. Other universal wave phenomena, such as propagation in parity-time symmetric systems, can be accessed and experimentally explored with the most convenient wave system yet the implications are broadly applicable.

A second direction is the development of materials and devices for controlling waves and transport in these different domains, including acoustics, elastodynamics, and heat flow. Interestingly, many of the challenges in implementing transformation materials and devices in all of these domains are similar 
to those in optics and electromagnetics. The transformation design approach typically yields continuum material parameters that are anisotropic and smoothly inhomogeneous, and these properties need to be controlled with a precision not normally available in natural materials.

The most successful solution to this challenge has been engineered metamaterials. Metamaterial and related design approaches have successfully been applied in a variety of wave and transport systems to experimentally demonstrate the feasibility of practical implementations of transformation designs [e.g. 7]. That said, improvements to metamaterial design and fabrication are still needed to carry these concepts beyond simple feasibility demonstrations towards practically useful devices.

\section{Advances in Science and Technology to Meet Challenges}

The instrincally multiscale design, simulation, and fabrication of metamaterials remains a major hurdle for the design of transformation-based devices for any wave system. Subwavelength scales are needed to mimic continuum materials, while the device-scale inhomogeneity is typically many orders of magnitude larger. Additive manufacturing is a promising path, but most the capabilities of most implementations at present are not sufficiently multiscale.

Another challenge across all transformationbased designs is the physical realization of the required range of continuum material properties. Different background materials impose different practical constraints. In airborne acoustics, for example, it is difficult for nonresonant structures to behave as though they are lighter or more compressible than air. In contrast, for underwater acoustics, it is challenging to create structures that are more than a few times more dense or stiff than water. The range of material parameters needed can be controlled to some degree through the transformation framework, and developing design approaches that merge transformation theory with practical material fabrication limits will likely be essential for successful devices in any wave system.

A particular advantage that acoustics offers to transformation-based design lies in the relatively slow propagation velocity and relatively long time scales of frequencies of interest. The acoustic metamaterial response can be controlled actively at microsecond to millisecond timescales [8], and this enables the ability to create local material behavior not easily implemented in other wave systems, including gain. One example that exploits this is demonstrating the ability to control exceptional points in parity-time symmetric systems [9]. The development of new active approaches to control local acoustic metamaterial response should enable acoustic analogues of very complex wave systems.
One practically important wave system that remains relatively unexplored is solid elastodynamics. Part of the challenge is that the transformation design approach leads to solid materials that exhibit so-called Willis coupling [10]. Although continuum solids do not have this property, microstructured materials can, provided they have the right kind of internal asymmetry or lattice structure. The practical importance of waves and vibrations in solids suggests that further theoretical and experimental developments in this area may have substantial impact.

\section{Concluding Remarks}

The power of the transformation design framework is not limited to optics and electromagnetics. It appears to be applicable, to at least some degree, to most if not all wave and transport systems. These include acoustic waves in fluids, heat conduction, elastodynamic waves in solids, and even quantum mechanical matter waves. Anisotropic and inhomogeneous effective material properties are the critical component in experimentally realizing the level of wave control the transformation framework promises. Designing and fabricating artificial metamaterials with subwavelength structure that spans tens to hundreds (or more) wavelengths in total size remains a primary technical challenge in all of these areas. When this is possible, truly arbitrary wave and transport control become feasible.

\section{References}

[1] Cummer S and Schurig D 2007 One path to acoustic cloaking New J. Phys 945

[2] Chen H and Chan C 2007 Acoustic cloaking in three dimensions using acoustic metamaterials Appl. Phys. Lett. 91183518

[3] Zhang S, Genov D, Sun C and Zhang X 2008 Cloaking of matter waves Phys. Rev. Lett. 100 123002

[4] Guenneau S, Amra C and Veynante D 2012 Transformation thermodynamics: cloaking and concentrating heat flux Opt. Exp. 20 8207-18

[5] Greenleaf A, Lassas M and Uhlmann G 2003 Anisotropic conductivities that cannot be detected by EIT Physiol. Meas. 24 413-9

[6] Leonhardt, U 2015 On cosmology in the laboratory Phil. Trans. R. Soc. A 37320140354

[7] Zigoneanu L, Popa B and Cummer S 2014 Threedimensional broadband omnidirectional acoustic ground cloak Nat. Mat. 13 352-5

[8] Popa B, Zigoneanu L and Cummer S 2013 Tunable active acoustic metamaterials Phys. Rev. B. 88 024303

[9] Shi C, Dubois M, Chen Y, Cheng L, Ramezani H, Wang $\mathrm{Y}$ and Zhang $\mathrm{X}$ Accessing the exceptional points of parity-time acoustics Nat. Comms. 711110 
[10] Milton G, Briane M and Willis J 2006 On cloaking for elasticity and physical equations with a transformation invariant form New J. Phys. 8248 